\title{
Some Recent Generalizations of the Classical Rigid Body Systems
}

\author{
Vladimir Dragovićc ${ }^{1,2}$. Borislav Gajić
}

Received: 20 November 2014 / Revised: 13 July 2016 / Accepted: 25 August 2016 /

Published online: 19 September 2016

(C) Institute for Mathematical Sciences (IMS), Stony Brook University, NY 2016

\begin{abstract}
Some recent generalizations of the classical rigid body systems are reviewed. The cases presented include dynamics of a heavy rigid body fixed at a point in three-dimensional space, the Kirchhoff equations of motion of a rigid body in an ideal incompressible fluid as well as their higher-dimensional generalizations.
\end{abstract}

Keywords Rigid body dynamics - Lax representation - Euler-Arnold equations · Algebro-geometric integration procedure · Baker-Akhiezer function · Grioli precession - Kirchhoff equations

Mathematics Subject Classification Primary 70E17 · 70E40 · 14H70; Secondary $70 \mathrm{E} 45 \cdot 70 \mathrm{H} 06$

\section{Contents}

1 Introduction . . . . . . . . . . . . . . . . . . . . . . . . 512

2 The Hess-Appel'rot Case of Rigid Body Motion . . . . . . . . . . . . . . . . . . . . . . . . . 514

2.1 Basic Notions of Heavy Rigid Body Fixed at a Point . . . . . . . . . . . . . . . . . . . . 514

2.2 Integrable Cases . . . . . . . . . . . . . . . . . . . . . . . . . 517

2.3 Definition of the Hess-Appel'rot System . . . . . . . . . . . . . . . . . . . . . . 517

Vladimir Dragović

vladimir.dragovic@utdallas.edu

Borislav Gajić

gajab@mi.sanu.ac.rs

1 The Department of Mathematical Sciences, The University of Texas at Dallas, Richardson, TX, USA

2 Mathematical Institute, Serbian Academy of Science and Art, Kneza Mihaila 36, 11000 Belgrade, Serbia 
2.4 A Lax Representation for the Classical Hess-Appel'rot System:

An Algebro-Geometric Integration Procedure . . . . . . . . . . . . . . . . . . 518

2.5 Zhukovski's Geometric Interpretation . . . . . . . . . . . . . . . . . . . . . . 521

3 Kowalevski Top, Discriminantly Separable Polynomials, and Two Valued Groups . . . . . . . . . 522

3.1 Discriminantly Separable Polynomials . . . . . . . . . . . . . . . . . . . . . 522

3.2 Two-Valued Groups . . . . . . . . . . . . . . . . . . . . . . . . . . . . . . . . . . . . . . 524

3.3 2-Valued Group Structure on $\mathbb{C P}^{1}$ and the Kowalevski Fundamental Equation . . . . . . . . 526

3.4 Fundamental Steps in the Kowalevski Integration Procedure . . . . . . . . . . . . . . . . . 527

3.5 Systems of the Kowalevski Type: Definition . . . . . . . . . . . . . . . . . . . . 531

3.6 An Example of Systems of the Kowalevski Type . . . . . . . . . . . . . . . . . . . . . 532

3.7 Another Example of an Integrable System of the Kowalevski Type . . . . . . . . . . . . . . 535

3.8 Another Class of Systems of the Kowalevski Type . . . . . . . . . . . . . . . . . . . . . . 537

3.9 A Deformation of the Kowalevski Top . . . . . . . . . . . . . . . . . . . . . . . 539

4 The Lagrange Bitop and the $n$-Dimensional Hess-Appel'rot Systems . . . . . . . . . . . . . . . 544

4.1 Higher-Dimensional Generalizations of Rigid Body Dynamics . . . . . . . . . . . . . . . . 544

4.2 The Heavy Rigid Body Equations on $e(n) \ldots \ldots \ldots$. . . . . . . . . . . . 546

4.3 The Heavy Rigid Body Equations on $s=s o(n) \times_{a d} s o(n) \ldots \ldots \ldots$. . . . . . . . . 547

4.4 Four-Dimensional Rigid Body Motion . . . . . . . . . . . . . . . . . . . . . . . . . . . . . 548

4.5 The Lagrange Bitop: Definition and a Lax Representation . . . . . . . . . . . . . . . . . . 549

4.5 .1 Classical Integration . . . . . . . . . . . . . . . . . . . . . . 551

4.5 .2 Properties of the Spectral Curve . . . . . . . . . . . . . . . . . . . 552

4.6 Four-Dimensional Hess-Appel'rot Systems . . . . . . . . . . . . . . . . . . . . . 554

4.7 The $n$-Dimensional Hess-Appel'rot Systems . . . . . . . . . . . . . . . . . . . 556

4.8 Classical Integration of the Four-Dimensional Hess-Appel'rot System . . . . . . . . . . . . 559

5 Four-Dimensional Grioli-Type Precessions . . . . . . . . . . . . . . . . . . . . . . . . . 562

5.1 The Classical Grioli Case . . . . . . . . . . . . . . . . . . . . . . . . . . 562

5.2 Four-Dimensional Grioli Case . . . . . . . . . . . . . . . . . . . . . . . . 564

6 Motion of a Rigid Body in an Ideal Fluid: The Kirchhoff Equations . . . . . . . . . . . . . . . 567

6.1 Integrable Cases . . . . . . . . . . . . . . . . . . . . . . . . . . 568

6.2 Three-Dimensional Chaplygin's Second Case . . . . . . . . . . . . . . . . . . . . 570

6.2 .1 Classical Integration Procedure . . . . . . . . . . . . . . . . . . . . 571

6.2.2 Lax Representation for the Chaplygin Case . . . . . . . . . . . . . . . . . . . . . . 572

6.3 Four-Dimensional Kirchhoff and Chaplygin Cases . . . . . . . . . . . . . . . . . . . 573

References . . . . . . . . . . . . . . . . . . . . . 575

\section{Introduction}

The rigid body dynamics is a core subject of the Classical Mechanics. Findings of Mathematics of the last 40-50 years, shed a new light on Mechanics, and on rigidbody dynamics in particular. Strong impetus to a novel approach and modernization of Mechanics came from celebrated Arnold's book (Arnold 1974). There are several approaches and schools developing further those ideas. To mention a few: Kozlov, Abraham, Marsden, Kharlamov, Manakov, Novikov, Dubrovin, Fomenko, Horozov, Sokolov and their students and collaborators. Along that line, in 1993, the first author initiated a new seminar in the Mathematical Institute of the Serbian Academy of Sciences and Arts, inspired by and named after Arnold's book. One of the most popular research topics within the Seminar was rigid body dynamics, and several young people got involved. The purpose of this article is to make a review of some of the results obtained in almost quarter of the century of the Seminar's activity. Let us mention that the classical aspects of rigid body dynamics occupied attention of Serbian scientists for a long time, see for example books and monographs (Bilimović 1955; Dragović and Milinković 2003; Andjelić and Stojanović 1966) and references therein. 
In this paper we will focus mostly on the problems related to the integrability of motion of a rigid body either in the case of a heavy body fixed at a point or a body embedded in an ideal fluid and their higher dimensional generalizations. Although the first higher dimensional generalizations of rigid body dynamics appeared in XIX century (see Frahm 1874; Schottky 1891), a strong development of the subject came after Arnold's paper (1966).

Integrability or solvability, is one of the fundamental questions related to the system of differential equations of motion of some mechanical system. The integrability is closely related to the existence of enough number of independent first integrals, i.e. functions that are constant along the solutions of the system. Early history was developed by classics (Euler, Lagrange, Hamilton, Jacobi, Liouville, Kowalevski, Poincaré, E. Noether, and many others). The basic method of that time was the method of separation of variables and Noether's theorem was the tool for finding first integrals from the symmetries of the system. With the work of Kowalevski a more subtle algebraic geometry and more intensive theory of theta functions entered on the stage. The final formulation of the principle theorem of the subject of classical integrability, the Liouville-Arnold theorem, which gives a qualitative picture of the integrable finitedimensional Hamiltonian systems appeared in the Arnold's paper (Arnold 1963) (see also Arnold 1974).

In the classical history of integration in rigid body dynamics, the paper of Kowalevski (1889) occupies a special place. For the previously known integrable examples, the Euler and the Lagrange case, the solutions are meromorphic functions. Starting from that observation, Kowalevski formulated the problem of finding all cases of rigid body motion fixed at a point whose general solutions are singlevalued functions of complex time that admit only moving poles as singularities. She proved that this was possible only in one additional case, named later the Kowalevski case. She found an additional first integral of fourth degree and completely solved the equations of motion in terms of genus two theta-functions. The importance of the Kowalevski paper is reflected in the number and spectra of papers that are devoted to the Kowalevski top. We will present here some recent progress in the geometric interpretation of the Kowalevski integration and certain generalizations of the Kowalevski top see (Dragović 2010; Dragović and Kukić 2011, 2014a, b).

A modern, algebro-geometric approach to integration of the equations of motion is based on the existence of the so-called Lax representations. This method originated in the 1960's, with a significant breakthrough made in the theory of integrable nonlinear partial differential equations (Korteveg-de Vries (KdV), Kadomtsev-Petviashvili (KP) and others). These equations appear to be integrable infinite-dimensional Hamiltonian systems. A system admits a Lax representation (or an L-A pair) with the spectral parameter if there exists a pair of linear operators (matrices, for example) $L(\lambda), A(\lambda)$ such that the equations of the system can be written in the form:

$$
\frac{d}{d t} L(\lambda)=[L(\lambda), A(\lambda)]
$$


where $\lambda$ is the complex spectral parameter. The first consequence of (1.1) is that the spectrum of the matrix $L(\lambda)$ is constant in time, i.e. the coefficients of the spectral polynomial are first integrals. In the algebro-geometric integration procedure, the so-called Baker-Akhiezer function plays a key role. This function is the common eigenfunction of the operators $\frac{d}{d t}+A(\lambda)$ and $L(\lambda)$, defined on the spectral curve $\mathcal{C}$, which is naturally associated to the L-A pair. The Baker-Akhiezer function is meromorphic on $\mathcal{C}$ except in several isolated points where it has essential singularities. For more detailed explanations of modern algebro-geometric integration methods, see (Dubrovin 1977, 1981; Dubrovin et al. 2001; Adler and van Moerbeke 1980; Dragović 2006; Belokolos et al. 1994; Gajić 2002). An important class of Lax presentations, when $L(\lambda)$ and $A(\lambda)$ are matrix polynomials in $\lambda$, was studied by Dubrovin (1977) (see also Dubrovin (1981); Dubrovin et al. (2001)). These methods have been successfully applied to rigid body dynamics (see Manakov 1976; Bogoyavlensky 1984; Bobenko et al. 1989; Ratiu and van Moerbeke 1982; Ratiu 1982; Gavrilov and Zhivkov 1998).

The Lax representations appear to be also a useful tool for constructing higherdimensional generalizations of a given system. We will review some of the results obtained in Dragović and Gajić (2001, 2004, 2006, 2009, 2012, 2014), Dragović (2010), Jovanović (2007, 2008), Gajić (2013).

The paper is organized as follows. The basic facts about three-dimensional motion of a rigid body are presented in Sect. 2. In the same Section, the basic steps of the algebro-geometric integration procedure for the Hess-Appel'rot case of motion of three-dimensional rigid body are given. A recent approach to the Kowalevski integration procedure is given in Sect. 3. The basic facts of higher-dimensional rigid body dynamics are presented in Sect. 4. The same Section provides the definition of the isoholomorphic systems, such as the Lagrange bitop and $n$-dimensional Hess-Appel'rot systems. The importance of the isoholmorphic systems has been underlined by Grushevsky and Krichever (2010). In Sect. 5 we review the classical Grioli precessions and present its quite recent higher-dimensional generalizations. The four-dimensional generalizations of the Kirchhoff and Chaplygin cases of motion of a rigid body in an ideal fluid are given in Sect. 6.

\section{The Hess-Appel'rot Case of Rigid Body Motion}

\subsection{Basic Notions of Heavy Rigid Body Fixed at a Point}

A three-dimensional rigid body is a system of material points in $\mathbb{R}^{3}$ such that the distance between each two points is a constant function of time. Important case of motion is when rigid body moves with fixed point $O$. Then the configuration space is the Lie group $S O(3)$. In order to describe the motion, it is usual to introduce two Euclidian frames associated to the system: the first one $O x y z$ is fixed in the space, and the second, moving, $O X Y Z$ is fixed in the body. The capital letters will denote elements of the moving reference frame, while the lowercase letters will denote elements of the fixed reference frame. Let $B(t) \in S O(3)$ is an orthogonal matrix which maps $O X Y Z$ to $O x y z$. The radius vector $\vec{Q}$ of the arbitrary point in the moving coordinate system maps to the radius vector in the fixed frame $\vec{q}(t)=B(t) \vec{Q}$. The 
velocity of that point in the fixed reference frame is given by

$$
\vec{v}(t)=\dot{\vec{q}}(t)=\dot{B}(t) \vec{Q}=\dot{B}(t) B^{-1}(t) \vec{q}(t)=\omega(t) \vec{q}(t),
$$

where $\omega(t)=\dot{B} B^{-1}$. The matrix $\omega$ is an skew-symmetric matrix. Using the isomorphism of $\left(\mathbb{R}^{3}, \times\right)$, where $\times$ is the usual vector product, and $(\operatorname{so}(3),[]$,$) , given$ by

$$
\vec{a}=\left(a_{1}, a_{2}, a_{3}\right) \mapsto a=\left[\begin{array}{ccc}
0 & -a_{3} & a_{2} \\
a_{3} & 0 & -a_{1} \\
-a_{2} & a_{1} & 0
\end{array}\right]
$$

matrix $\omega(t)$ is corresponded to vector $\vec{\omega}(t)$-angular velocity of the body in the fixed reference frame. Then $\vec{v}(t)=\vec{\omega}(t) \times \vec{q}(t)$. One can easily see that $\vec{\omega}(t)$ is the eigenvector of matrix $\omega(t)$ that corresponds to the zero eigenvalue.

In the moving reference frame, $\vec{V}(t)=B(t)^{-1} \vec{v}(t)$, so $\vec{V}(t)=\vec{\Omega}(t) \times \vec{Q}$, where $\vec{\Omega}(t)$ is the angular velocity in the moving reference frame and corresponds to the skew-symmetric matrix $\Omega(t)=B^{-1}(t) \dot{B}(t)$.

Here one concludes that it is natural to consider the angular velocity as a skewsymmetric matrix. The element $\omega_{12}$ corresponds to the rotation in the plane determined by the first two axes $O x$ and $O y$, and similarly for the other elements. In the threedimensional case we have a natural correspondence given above, and one can consider the angular velocity as a vector. But, in higher-dimensional cases, generally speaking, such a correspondence does not exist. We will see later how in dimension four, using isomorphism between $s o(4)$ and $s o(3) \times s o(3)$ two vectors in the three-dimensional space are joined to an $4 \times 4$ skew-symmetric matrix.

The moment of inertia with respect to the axis $u$, defined with the unit vector $\vec{u}$ through a fixed point $O$ is :

$$
I(u)=\int_{B} d^{2} d m=\int_{B}\langle\vec{u} \times \vec{Q}, \vec{u} \times \vec{Q}\rangle d m=\int_{B}\langle\vec{Q} \times(\vec{u} \times \vec{Q}), \vec{u}\rangle d m=\langle I \vec{u}, \vec{u}\rangle,
$$

where $d$ is the distance between corresponding point and axis $u, I$ is inertia operator with respect to the point $O$ defined with

$$
I \vec{u}=\int_{B} \vec{Q} \times(\vec{u} \times \vec{Q}) d m
$$

and integrations goes over the body $B$. The diagonal elements $I_{1}, I_{2}, I_{3}$ are called the principal moments of inertia, with respect to the principal axes of inertia. The ellipsoid $\langle I \Omega, \Omega\rangle=1$ is the inertia ellipsoid of the body at the point $O$. In the principal coordinates its equation is:

$$
I_{1} \Omega_{1}^{2}+I_{2} \Omega_{2}^{2}+I_{3} \Omega_{3}^{2}=1
$$


The kinetic energy of the body is given by:

$$
\begin{aligned}
T & =\frac{1}{2} \int_{B} V^{2} d m=\frac{1}{2} \int_{B}\langle\vec{\Omega} \times \vec{Q}, \vec{\Omega} \times \vec{Q}\rangle d m \\
& =\frac{1}{2}\left\langle\vec{\Omega}, \int_{B} \vec{Q} \times(\vec{\Omega} \times \vec{Q}) d m\right\rangle=\frac{1}{2}\langle I \vec{\Omega}, \vec{\Omega}\rangle
\end{aligned}
$$

Similarly, for the angular momentum $\vec{M}$ with respect to the point $O$, we have:

$$
\vec{M}=\int_{B} \vec{Q} \times \vec{V} d m=\int_{B} \vec{Q} \times(\vec{\Omega} \times \vec{Q}) d m=I \vec{\Omega} .
$$

We consider a motion of a heavy rigid body fixed at a point. Let us denote by $\vec{\chi}$ the radius vector of the center of masses of the body multiplied with the mass $m$ of the body and the gravitational acceleration $g$. By $\vec{\Gamma}$ we denote the unit vertical vector.

The motion in the moving reference frame is described by the Euler-Poisson equations (Leimanis 1965; Whittaker 1952; Golubev 1953; Borisov and Mamaev 2001):

$$
\begin{aligned}
\dot{\vec{M}} & =\vec{M} \times \vec{\Omega}+\vec{\Gamma} \times \vec{\chi} \\
\dot{\vec{\Gamma}} & =\vec{\Gamma} \times \vec{\Omega} .
\end{aligned}
$$

Using that $\vec{M}=I \vec{\Omega}$, one see that (2.2) as a system of six ordinary differential equations in $\vec{M}$ and $\vec{\Gamma}$ with six parameters $I=\operatorname{diag}\left(I_{1}, I_{2}, I_{3}\right), \vec{\chi}=\left(X_{0}, Y_{0}, Z_{0}\right)$. These equations have three first integrals:

$$
\begin{aligned}
H & =\frac{1}{2}\langle\vec{M}, \vec{\Omega}\rangle+\langle\vec{\Gamma}, \vec{\chi}\rangle \\
F_{1} & =\langle\vec{M}, \vec{\Gamma}\rangle \\
F_{2} & =\langle\vec{\Gamma}, \vec{\Gamma}\rangle .
\end{aligned}
$$

Since the equations preserve the standard measure, by the Jacobi theorem (see for example Golubev 1953; Arnold et al. 2009) for integrability in quadratures one needs one more additional functionally independent first integral.

On the other hand, the Eq. (2.2) are Hamiltonian on the Lie algebra $e(3)$ with the standard Lie-Poisson structure:

$$
\left\{M_{i}, M_{j}\right\}=-\epsilon_{i j k} M_{k}, \quad\left\{M_{i}, \Gamma_{j}\right\}=-\epsilon_{i j k} \Gamma_{k}, \quad i, j, k=1,2,3 .
$$

The structure (2.4) has two Casimir functions $F_{1}$ and $F_{2}$ from (2.3). Thus, the symplectic leaves are four-dimensional (they are diffeomorphic to the cotangent bundle of the two-dimensional sphere (Kozlov 1995) and for the integrability in the Liouville sense one needs, besides the Hamiltonian $H$ from (2.3), one more functionally independent first integral.

Thus a natural problem arises: for which values of the parameters $I_{1}, I_{2}, I_{3}$, $X_{0}, Y_{0}, Z_{0}$, the Eq. (2.2) admit the fourth functionally independent first integral? 


\subsection{Integrable Cases}

The existence of an additional independent fourth integral gives strong restrictions on the moments of inertia and the vector $\vec{\chi}$. Such an integral exists in the three cases (Euler 1765; Lagrange 1788; Kowalevski 1889) (see also Golubev 1953; Leimanis 1965; Whittaker 1952; Kozlov 1995; Borisov and Mamaev 2001; Arkhangel'skiy 1977):

- Euler case (1751): $X_{0}=Y_{0}=Z_{0}=0$. The additional integral is $F_{4}=\langle M, M\rangle$.

- Lagrange case (1788): $I_{1}=I_{2}, \vec{\chi}=\left(0,0, Z_{0}\right)$. The additional integral is $F_{4}=$ $\mathrm{M}_{3}$.

- Kovalewski case (1889): $I_{1}=I_{2}=2 I_{3}, \vec{\chi}=\left(X_{0}, 0,0\right)$. The additional integral is $F_{4}=\left(\Omega_{1}^{2}-\Omega_{2}^{2}+\frac{X_{0}}{I_{3}} \Gamma_{1}\right)^{2}+\left(2 \Omega_{1} \Omega_{2}+\frac{X_{0}}{I_{3}} \Gamma_{2}\right)^{2}$.

There are also cases that admit a fourth first integral only for a fixed value of one of the integrals. If the Casimir function $F_{1}=0$, then we have

- Goryachev-Chaplygin case (1900): $I_{1}=I_{2}=4 I_{3}, \vec{\chi}=\left(X_{0}, 0,0\right)$. The additional integral is $F_{4}=M_{3}\left(M_{1}^{2}+M_{2}^{2}\right)+2 M_{1} \Gamma_{3}$;

Following the Kowalevski paper (1889), a natural problem arises: to find all cases of the Euler-Poisson equations that admit an additional fourth first integral. Using the results of Liouville, in Husson (1906) Husson proved that an additional algebraic integral exists only in the Euler, Lagrange and Kovalewski cases. Simplified proofs of Liouville's and Husson's results were presented by Dokshevich (see Dokshevich 1974). On the other hand, Poincaré considered a more general problem of the existence of an analytical first integral of the canonical systems. Using the method of a small parameter, he developed a tool for proving nonintegrability of a perturbation of an integrable Hamiltonian system. However, Poincaré observed that his method cannot be applied to the Euler-Poisson equations. In 1970's Kozlov in Kozlov (1975) (see also Kozlov 1980; Arkhangel'skiy 1977) modified the Poincaré results and proved that a nonsymmetric rigid body does not admit an additional analytical integral except in the Euler case. The case of a symmetric rigid body is even more complicated. The nonexistence of an additional (complex or real-valued) analytical or meromorphic integral except in the three classical cases was finally proved in the papers of Kozlov and Treschev (1985, 1986), Ziglin (1997). Ziglin also proved that having the value of $F_{1}$ fixed to be zero, an additional meromorphic integral exists only in one extra case-the Goryachev-Chaplygin case.

\subsection{Definition of the Hess-Appel'rot System}

Beside the completely integrable cases, there are classically well-known systems which possess an invariant relation instead of an additional first integral. A list of such systems can be found, for example in Gorr et al. (1978).

Some of these cases where obtained using new forms of the Euler-Poisson equations and method of constructing invariant relations given by Kharlamov (for details see Kharlamov 1965, 1974a,b; Kharlamov and Kovalev 1997; Gorr et al. 1978; Gashenenko et al. 2012). We will focus on the Hess-Appel'rot case. 
It is well known that Kowalevski, in her above mentioned celebrated 1889 paper Kowalevski (1889), started with a careful analysis of the solutions of the Euler and the Lagrange case of rigid-body motion, and formulated a problem to describe the parameters $\left(I_{1}, I_{2}, I_{3}, X_{0}, Y_{0}, Z_{0}\right)$, for which the Euler-Poisson equations have a general solution in a form of a uniform (single-valued) function having moving poles as the only possible singularities.

Then, some necessary conditions were formulated in Kowalevski (1889) and a new case was discovered, now known as the Kowalevski case, as a unique possible beside the cases of Euler and Lagrange. However, considering the situation where all the momenta of inertia are different, Kowalevski came to a relation analogue to the following:

$$
X_{0} \sqrt{I_{1}\left(I_{2}-I_{3}\right)}+Y_{0} \sqrt{I_{2}\left(I_{3}-I_{1}\right)}+Z_{0} \sqrt{I_{3}\left(I_{1}-I_{2}\right)}=0
$$

and concluded that the relation $X_{0}=Y_{0}=Z_{0}$ follows, leading to the Euler case.

But, it was Appel'rot (see Appel'rot 1892, 1894) who noticed in the beginning of 1890 's, that the last relation admitted one more case, not mentioned by Kowalevski:

$$
Y_{0}=0, \quad X_{0} \sqrt{I_{1}\left(I_{2}-I_{3}\right)}+Z_{0} \sqrt{I_{3}\left(I_{1}-I_{2}\right)}=0
$$

under the assumption $I_{1}>I_{2}>I_{3}$. Such an intriguing position corresponding to the overlook in the Kowalevski paper, made the Hess-Appel'rot systems very attractive for leading Russian mathematicians from the end of XIX century. Nekrasov and Lyapunov managed to provide new arguments and they demonstrated that the Hess-Appel'rot systems didn't satisfy the condition investigated by Kowalevski, which meant that her conclusion was correct. It is interesting to mention that in Appel'rot (1892), Appel'rot noticed that the first version of his paper had a mistake observed and communicated to him by Nekrasov.

The system that satisfies the conditions (2.5) was considered also by Hess, even before Appel'rot, in 1890. Hess (1890) found that if the inertia momenta and the radius vector of the center of masses satisfy the conditions (2.5), then the surface

$$
F_{4}=M_{1} X_{0}+M_{3} Z_{0}=0
$$

is invariant. It means that if at the initial moment the relation $F_{4}=0$ is satisfied, then it will be satisfied during the whole time evolution of the system.

\subsection{A Lax Representation for the Classical Hess-Appel'rot System: An Algebro-Geometric Integration Procedure}

A Lax representation for the classical Hess-Appel'rot system, with an algebrogeometric integration procedure was presented in Dragović and Gajić (2001). The classical integration procedure leads to an elliptic function and an additional Riccati equation (see Golubev 1953). In Dragović and Gajić (2001) an algebro-geometric integration procedure was presented with the same properties. 
Using isomorphism (2.1), Eq. (2.2) can be written in the matrix form:

$$
\begin{aligned}
\dot{M} & =[M, \Omega]+[\Gamma, \chi] \\
\dot{\Gamma} & =[\Gamma, \Omega],
\end{aligned}
$$

where the skew-symmetric matrices represent vectors denoted by the same letter.

We have the following:

Theorem 2.1 (Dragović and Gajić 2001) If condition (2.5) is satisfied, the equations of the Hess-Appel'rot case can be written in the form:

$$
\begin{aligned}
& \dot{L}(\lambda)=[L(\lambda), A(\lambda)], \\
& L(\lambda)=\lambda^{2} C+\lambda M+\Gamma, \quad A(\lambda)=\lambda \chi+\Omega, \quad C=I_{2} \chi .
\end{aligned}
$$

The spectral curve is defined by:

$$
\mathcal{C}: p(\mu, \lambda):=\operatorname{det}(L(\lambda)-\mu E)=0,
$$

is:

$$
\mathcal{C}:-\mu\left(\mu^{2}-\omega^{2}+2 \Delta \Delta^{*}\right)=0
$$

where

$$
\begin{aligned}
\alpha & =\frac{X_{0}}{\sqrt{X_{0}^{2}+Z_{0}^{2}}} \quad \beta=\frac{Z_{0}}{\sqrt{X_{0}^{2}+Z_{0}^{2}}} \\
\Delta & =y+\lambda x, \quad \Delta^{*}=\bar{y}+\lambda \bar{x} \\
y & =\frac{1}{\sqrt{2}}\left(\beta \Gamma_{1}-\alpha \Gamma_{3}-i \Gamma_{2}\right), \quad x=\frac{1}{\sqrt{2}}\left(\beta M_{1}-\alpha M_{3}-i M_{2}\right), \\
\omega & =-i\left[\alpha\left(C_{1} \lambda^{2}+M_{1} \lambda+\Gamma_{1}\right)+\beta\left(C_{3} \lambda^{2}+M_{3} \lambda+\Gamma_{3}\right)\right] \\
& =-i\left[\alpha\left(C_{1} \lambda^{2}+\Gamma_{1}\right)+\beta\left(C_{3} \lambda^{2}+\Gamma_{3}\right)\right] .
\end{aligned}
$$

This curve is reducible. It consists of two components: the rational curve $\mathcal{C}_{1}$ given by $\mu=0$, and the elliptic curve $\mathcal{C}_{2}$ :

$$
\mu^{2}=P_{4}(\lambda)=\omega^{2}-2 \Delta \Delta^{*}
$$

The coefficients of the spectral polynomial are integrals of motion. If one rewrites the equation of the spectral curve in the form:

$$
p(\mu, \lambda)=-\mu\left(\mu^{2}+A \lambda^{4}+B \lambda^{3}+D \lambda^{2}+E \lambda+F\right)=0,
$$


one gets:

$$
\begin{aligned}
& A=I_{2}^{2}\left(X_{0}^{2}+Z_{0}^{2}\right), \\
& B=2 I_{2}\left(M_{1} X_{0}+M_{3} Z_{0}\right)(=0), \\
& D=M_{1}^{2}+M_{2}^{2}+M_{3}^{2}+2 I_{2}\left(X_{0} \Gamma_{1}+Z_{0} \Gamma_{3}\right), \\
& E=2\left(M_{1} \Gamma_{1}+M_{2} \Gamma_{2}+M_{3} \Gamma_{3}\right), \\
& F=\Gamma_{1}^{2}+\Gamma_{2}^{2}+\Gamma_{3}^{2}(=1) .
\end{aligned}
$$

Thus, the L-A pair (2.7) gives three first integrals and one invariant relation.

Now, we review some basic steps in the algebro-geometric integration procedure from Dragović and Gajić (2001). Let $\left(f_{1}, f_{2}, f_{3}\right)^{T}$ denote an eigenvector of the matrix $L(\lambda)$, which corresponds to the eigenvalue $\mu$. Fix the normalizing condition $f_{1}=1$. Then one can prove:

Lemma 2.1 (Dragović and Gajić 2001) The divisors of $f_{2}$ and $f_{3}$ on $\mathcal{C}_{2}$ are:

$$
\begin{aligned}
& \left(f_{2}\right)=-P_{1}+P_{2}-v+\bar{v}, \\
& \left(f_{3}\right)=P_{1}-P_{2}+v-\bar{v},
\end{aligned}
$$

where $P_{1}$ and $P_{2}$ are points on $\mathcal{C}_{2}$ over $\lambda=\infty$, and $v \in \mathcal{C}_{2}$ is defined with $\nu_{\lambda}=-\frac{y}{x}$, $v_{\mu}=-\left.\omega\right|_{\lambda=-\frac{y}{x}}$.

We are going to analyze the converse problem. Suppose the evolution in time of the point $v$ is known. For reconstructing the matrix $L(\lambda)$, one needs $x=|x| e^{i \arg x}, y=$ $|y| e^{i \arg y}$ as functions of time.

Lemma 2.2 (Dragović and Gajić 2001) The point $v \in \Gamma_{2}$ and the initial conditions for $M$ and $\Gamma$ determine $|x|,|y|$ and $\arg y-\arg x$, where $x$ and $y$ are given by (2.8).

Thus, in order to determine $L(\lambda)$ as a function of time, one needs to find the evolution of the point $v$ and $\arg x$ as a function of time. In Dragović and Gajić (2001) the following two theorems are proved:

Theorem 2.2 (Dragović and Gajić 2001) The integration of the motion of the point $v$ reduces to the inversion of the elliptical integral

$$
\int_{\nu_{0}}^{\nu} \frac{d \lambda}{\sqrt{\omega^{2}-2 \Delta \Delta^{*}}}=\frac{1}{I_{2}} t .
$$

Denote by $\phi_{x}=\arg x$, and $u=\tan \frac{\phi_{x}}{2}$.

Theorem 2.3 (Dragović and Gajić 2001) The function u( $t$ ) satisfies the Riccati equation:

$$
\frac{d u}{d t}=[f(t)+g(t)] u^{2}+[f(t)-g(t)]
$$


where

$$
\begin{aligned}
f(t) & =\frac{K}{2|x|^{2}}, \quad g(t)=\frac{Q|x|}{2}, \\
K & =\frac{\langle M, \Gamma\rangle}{2 \sqrt{X_{0}^{2}+Z_{0}^{2}}}, \quad Q=\frac{\beta}{\alpha} \sqrt{2}\left(\frac{1}{I_{2}}-\frac{1}{I_{1}}\right) ;
\end{aligned}
$$

$|x|$ is a known function of time.

In recent years some other methods have been applied as well to study the HessAppel'rot system (see Borisov and Mamaev 2003; Lubowiecki and Żołądek 2012a, b; Belyaev 2015; Simić 2000).

\subsection{Zhukovski's Geometric Interpretation}

In Zhukovski (1894) Zhukovski gave a geometric interpretation of the Hess-Appel'rot conditions. Denote $J_{i}=1 / I_{i}$. Consider the so-called gyroscopic inertia ellipsoid:

$$
\frac{M_{1}^{2}}{J_{1}}+\frac{M_{2}^{2}}{J_{2}}+\frac{M_{3}^{2}}{J_{3}}=1
$$

and the plane containing the middle axis and intersecting the ellipsoid at a circle. Denote by $l$ the normal to the plane, which passes through the fixed point $O$. Then the condition (2.5) means that the center of masses lies on the line $l$.

If we choose a basis of moving frame such that the third axis is $l$, the second one is directed along the middle axis of the ellipsoid, and the first one is chosen according to the orientation of the orthogonal frame, then (see Borisov and Mamaev 2001), the invariant relation (2.6) becomes

$$
F_{4}=M_{3}=0,
$$

the matrix $J$ obtains the form:

$$
J=\left(\begin{array}{ccc}
J_{1} & 0 & J_{13} \\
0 & J_{1} & 0 \\
J_{13} & 0 & J_{3}
\end{array}\right)
$$

and $\chi=\left(0,0, Z_{0}\right)$.

One can see here that the Hess-Appel'rot system can be regarded as a perturbation of the Lagrange top. In new coordinates the Hamiltonian of the Hess-Appel'rot system becomes

$$
H_{H A}=\frac{1}{2}\left(J_{1}\left(M_{1}^{2}+M_{2}^{2}\right)+J_{3} M_{3}^{2}\right)+Z_{0} \Gamma_{3}+J_{13} M_{1} M_{3}=H_{L}+J_{13} M_{1} M_{3}
$$

This serves as a motivation for a definition of higher-dimensional Hess-Appel'rot systems in Dragović and Gajić (2006), which will be presented in Sect. 4.6. 


\section{Kowalevski Top, Discriminantly Separable Polynomials, and Two Valued Groups}

We will present here a recent approach to a geometrization of the Kowalevski integration procedure from Dragović (2010), see also Dragović (2014).

\subsection{Discriminantly Separable Polynomials}

We will start from the equation of a pencil of conics $\mathcal{F}\left(w, x_{1}, x_{2}\right)=0$, where $w, x_{1}$ and $x_{2}$ are the pencil parameter and the Darboux coordinates respectively. We recall some of the details: given two conics $C_{1}$ and $C_{2}$ in general position by their tangential equations

$$
\begin{aligned}
& C_{1}: a_{0} w_{1}^{2}+a_{2} w_{2}^{2}+a_{4} w_{3}^{2}+2 a_{3} w_{2} w_{3}+2 a_{5} w_{1} w_{3}+2 a_{1} w_{1} w_{2}=0 ; \\
& C_{2}: w_{2}^{2}-4 w_{1} w_{3}=0 .
\end{aligned}
$$

Then the conics of this general pencil $C(s):=C_{1}+s C_{2}$ share four common tangents. The coordinate equations of the conics of the pencil are

$$
F\left(s, z_{1}, z_{2}, z_{3}\right):=\operatorname{det} M\left(s, z_{1}, z_{2}, z_{3}\right)=0,
$$

where the matrix $M$ is:

$$
M\left(s, z_{1}, z_{2}, z_{3}\right)=\left[\begin{array}{cccc}
0 & z_{1} & z_{2} & z_{3} \\
z_{1} & a_{0} & a_{1} & a_{5}-2 s \\
z_{2} & a_{1} & a_{2}+s & a_{3} \\
z_{3} & a_{5}-2 s & a_{3} & a_{4}
\end{array}\right] .
$$

The point equation of the pencil $C(s)$ is then of the form of the quadratic polynomial in $s$

$$
F:=H+K s+L s^{2}=0
$$

where $H, K$ and $L$ are quadratic expressions in $\left(z_{1}, z_{2}, z_{3}\right)$.

Given the projective plane with the standard coordinates $\left(z_{1}: z_{2}: z_{3}\right)$, we rationally parameterize the conic $C_{2}$ by $\left(1, \ell, \ell^{2}\right)$ as above. The tangent line to the conic $C_{2}$ through a point of the conic with the parameter $\ell_{0}$ is given by the equation

$$
t_{C_{2}}\left(\ell_{0}\right): z_{1} \ell_{0}^{2}-2 z_{2} \ell_{0}+z_{3}=0 \text {. }
$$

For a given point $\mathrm{P}$ outside the conic in the plane with coordinates $P=\left(\hat{z}_{1}, \hat{z}_{2}, \hat{z}_{3}\right)$, there are two corresponding solutions $x_{1}$ and $x_{2}$ of the equation quadratic in $\ell$

$$
\hat{z}_{1} \ell^{2}-2 \hat{z}_{2} \ell+\hat{z}_{3}=0 \text {. }
$$


Each of the solutions corresponds to a tangent to the conic $C_{2}$ from the point $P$. We will use the pair $\left(x_{1}, x_{2}\right)$ as the Darboux coordinates (see Darboux 1917) of the point $P$. One finds immediately the converse formulae

$$
\hat{z}_{1}=1, \quad \hat{z}_{2}=\frac{x_{1}+x_{2}}{2}, \quad \hat{z}_{3}=x_{1} x_{2} .
$$

Changing the variables in the polynomial $F$ from the projective coordinates $\left(z_{1}\right.$ : $\left.z_{2}: z_{3}\right)$ to the Darboux coordinates, we rewrite its equation $F$ in the form

$$
F\left(s, x_{1}, x_{2}\right)=L\left(x_{1}, x_{2}\right) s^{2}+K\left(x_{1}, x_{2}\right) s+H\left(x_{1}, x_{2}\right) .
$$

The key algebraic property of the pencil equation written in this form, as a quadratic equation in each of three variables $s, x_{1}, x_{2}$ is: all three of its discriminants are expressed as products of two polynomials in one variable each:

$$
\mathcal{D}_{s}(F)\left(x_{1}, x_{2}\right)=P\left(x_{1}\right) P\left(x_{2}\right), \quad \mathcal{D}_{x_{i}}(F)\left(s, x_{j}\right)=J(s) P\left(x_{j}\right), \quad i, j=1,2,
$$

where $\mathrm{J}$ and $\mathrm{P}$ are polynomials of degree 3 and 4 respectively, and the elliptic curves

$$
\Gamma_{1}: y^{2}=P(x), \quad \Gamma_{2}: y^{2}=J(s)
$$

are isomorphic (see Proposition 1 of Dragović 2010).

As a geometric interpretation of $F\left(s, x_{1}, x_{2}\right)=0$ we may say that the point $P$ in the plane, with the Darboux coordinates with respect to $C_{2}$ equal to $\left(x_{1}, x_{2}\right)$ belongs to two conics of the pencil, with the pencil parameters equal to $s_{1}$ and $s_{2}$, such that

$$
F\left(s_{i}, x_{1}, x_{2}\right)=0, \quad i=1,2 .
$$

Now we recall a general definition of the discriminantly separable polynomials. With $\mathcal{P}_{m}^{n}$ denote the set of all polynomials of $m$ variables of degree $n$ in each variable.

Definition 3.1 (Dragović 2010) A polynomial $F\left(x_{1}, \ldots, x_{n}\right)$ is discriminantly separable if there exist polynomials $f_{i}\left(x_{i}\right)$ such that for every $i=1, \ldots, n$

$$
\mathcal{D}_{x_{i}} F\left(x_{1}, \ldots, \hat{x}_{i}, \ldots, x_{n}\right)=\prod_{j \neq i} f_{j}\left(x_{j}\right) .
$$

It is symmetrically discriminantly separable if

$$
f_{2}=f_{3}=\cdots=f_{n}
$$

while it is strongly discriminantly separable if

$$
f_{1}=f_{2}=f_{3}=\cdots=f_{n}
$$


It is weakly discriminantly separable if there exist polynomials $f_{i}^{j}\left(x_{i}\right)$ such that for every $i=1, \ldots, n$

$$
\mathcal{D}_{x_{i}} F\left(x_{1}, \ldots, \hat{x}_{i}, \ldots, x_{n}\right)=\prod_{j \neq i} f_{j}^{i}\left(x_{j}\right) .
$$

\subsection{Two-Valued Groups}

\section{n-Valued Groups: Defining Notions}

The structure of formal (local) $n$-valued groups was introduced by Buchstaber and Novikov (1971) in their study of characteristic classes of vector bundles. It has been studied further by Buchstaber and his collaborators since then (see Buchstaber 2006 and references therein).

Following Buchstaber (2006), we give the definition of an $n$-valued group on $X$ as a map:

$$
\begin{gathered}
m: X \times X \rightarrow(X)^{n} \\
m(x, y)=x * y=\left[z_{1}, \ldots, z_{n}\right],
\end{gathered}
$$

where $(X)^{n}$ denotes the symmetric $n$-th power of $X$ and $z_{i}$ coordinates therein.

Associativity is the condition of equality of two $n^{2}$-sets

$$
\begin{aligned}
& {\left[x *(y * z)_{1}, \ldots, x *(y * z)_{n}\right]} \\
& {\left[(x * y)_{1} * z, \ldots,(x * y)_{n} * z\right]}
\end{aligned}
$$

for all triplets $(x, y, z) \in X^{3}$.

An element $e \in X$ is a unit if

$$
e * x=x * e=[x, \ldots, x]
$$

for all $x \in X$.

A map inv : $X \rightarrow X$ is an inverse if it satisfies

$$
e \in \operatorname{inv}(x) * x, \quad e \in x * \operatorname{inv}(x)
$$

for all $x \in X$.

Following Buchstaber, we say that $m$ defines an $n$-valued group structure $(X, m, e$, inv) if it is associative, with a unit and an inverse.

An $n$-valued group $X$ acts on the set $Y$ if there is a mapping

$$
\begin{gathered}
\phi: X \times Y \rightarrow(Y)^{n} \\
\phi(x, y)=x \circ y
\end{gathered}
$$


such that the two $n^{2}$-multisubsets of $Y$

$$
x_{1} \circ\left(x_{2} \circ y\right) \quad\left(x_{1} * x_{2}\right) \circ y
$$

are equal for all $x_{1}, x_{2} \in X, y \in Y$. It is additionally required that

$$
e \circ y=[y, \ldots, y]
$$

for all $y \in Y$.

Example 3.1 (A two-valued group structure on $\mathbb{Z}_{+}$, Buchstaber and Veselov 1996) Let us consider the set of nonnegative integers $\mathbb{Z}_{+}$and define a mapping

$$
\begin{gathered}
m: \mathbb{Z}_{+} \times \mathbb{Z}_{+} \rightarrow\left(\mathbb{Z}_{+}\right)^{2}, \\
m(x, y)=[x+y,|x-y|] .
\end{gathered}
$$

This mapping provides a structure of a two-valued group on $\mathbb{Z}_{+}$with the unit $e=0$ and the inverse equal to the identity $\operatorname{inv}(x)=x$.

In Buchstaber and Veselov (1996) a sequence of two-valued mappings associated with the Poncelet porism was identified as the algebraic representation of this 2-valued group. Moreover, the algebraic action of this group on $\mathbb{C P}^{1}$ was studied and it was shown that in the irreducible case all such actions are generated by the Euler-Chasles correspondences.

In the sequel, we are going to show that there is another 2-valued group and its action on $\mathbb{C P}^{1}$ which is even more closely related to the Euler-Chasles correspondence and to the Great Poncelet Theorem (see Dragović and Radnović 2011), and which is at the same time intimately related to the Kowalevski fundamental equation and to the Kowalevski change of variables.

However, we will start our approach with a simple example.

\section{The Simplest Case: 2-Valued Group $p_{2}$}

Among the basic examples of multivalued groups, there are $n$-valued additive group structures on $\mathbb{C}$. For $n=2$, this is a two-valued group $p_{2}$ defined by the relation

$$
\begin{gathered}
m_{2}: \mathbb{C} \times \mathbb{C} \rightarrow(\mathbb{C})^{2} \\
x *_{2} y=\left[(\sqrt{x}+\sqrt{y})^{2},(\sqrt{x}-\sqrt{y})^{2}\right]
\end{gathered}
$$

The product $x * 2 y$ corresponds to the roots in $z$ of the polynomial equation

$$
p_{2}(z, x, y)=0
$$

where

$$
p_{2}(z, x, y)=(x+y+z)^{2}-4(x y+y z+z x) .
$$

Our starting point in this section is the following 
Lemma 3.1 The polynomial $p_{2}(z, x, y)$ is discriminantly separable. The discriminants satisfy relations

$\mathcal{D}_{z}\left(p_{2}\right)(x, y)=P(x) P(y) \quad \mathcal{D}_{x}\left(p_{2}\right)(y, z)=P(y) P(z) \quad D_{y}\left(p_{2}\right)(x, z)=P(x) P(z)$, where $P(x)=2 x$.

The polynomial $p_{2}$ as a discriminantly separable, generates a case of generalized Kowalevski system of differential equations from Dragović (2010).

\subsection{2-Valued Group Structure on $\mathbb{C P}^{1}$ and the Kowalevski Fundamental Equation}

Now we pass to the general case. We are going to show that the general pencil equation represents an action of a two valued group structure. Recognition of this structure enables us to give to 'the mysterious Kowalevski change of variables' (see Audin 1996 for the wording "mysterious") a final algebro-geometric expression and explanation, developing further the ideas of Weil and Jurdjevic (see Weil 1983; Jurdjevic 1999a, b). Amazingly, the associativity condition for this action from geometric point of view is nothing else than the Great Poncelet Theorem for a triangle.

As we have already mentioned, the general pencil equation

$$
F\left(s, x_{1}, x_{2}\right)=0
$$

is connected with two isomorphic elliptic curves

$$
\begin{gathered}
\tilde{\Gamma}_{1}: y^{2}=P(x) \\
\tilde{\Gamma}_{2}: t^{2}=J(s)
\end{gathered}
$$

where the polynomials $P, J$ of degree four and three respectively. Suppose that the cubic one $\tilde{\Gamma}_{2}$ is rewritten in the canonical form

$$
\tilde{\Gamma}_{2}: t^{2}=J^{\prime}(s)=4 s^{3}-g_{2} s-g_{3}
$$

Moreover, denote by $\psi: \tilde{\Gamma}_{2} \rightarrow \tilde{\Gamma}_{1}$ a birational morphism between the curves induced by a fractional-linear transformation $\hat{\psi}$ which maps three zeros of $J^{\prime}$ and $\infty$ to the four zeros of the polynomial $P$.

The curve $\tilde{\Gamma}_{2}$ as a cubic curve has the group structure. Together with its subgroup $\mathbb{Z}_{2}$ it defines the standard two-valued group structure of coset type on $\mathbb{C P}^{1}$ (see Buchstaber 1990):

$$
s_{1} *_{c} s_{2}=\left[-s_{1}-s_{2}+\left(\frac{t_{1}-t_{2}}{2\left(s_{1}-s_{2}\right)}\right)^{2},-s_{1}-s_{2}+\left(\frac{t_{1}+t_{2}}{2\left(s_{1}-s_{2}\right)}\right)^{2}\right],
$$

where $t_{i}=J^{\prime}\left(s_{i}\right), i=1,2$. 
Theorem 3.1 Dragović (2010) The general pencil equation after fractional-linear transformations

$$
F\left(s, \hat{\psi}^{-1}\left(x_{1}\right), \quad \hat{\psi}^{-1}\left(x_{2}\right)\right)=0
$$

defines the two valued coset group structure $\left(\tilde{\Gamma}_{2}, \mathbb{Z}_{2}\right)$ defined by the relation (3.3).

For the proof see Dragović (2010).

\subsection{Fundamental Steps in the Kowalevski Integration Procedure}

Let us recall briefly that the Kowalevski top Kowalevski (1889) is a heavy top rotating about a fixed point, under the conditions $I_{1}=I_{2}=2 I_{3}, I_{3}=1, Y_{0}=Z_{0}=0$ (see Sect. 2.1). Denote with $c=m g X_{0}$, ( $m$ is the mass of the top), and with ( $\left.p, q, r\right)$ the vector of angular velocity $\vec{\Omega}$. Then the equations of motion take the following form, see Kowalevski (1889), Golubev (1953):

$$
\begin{aligned}
2 \dot{p} & =q r \quad \dot{\Gamma}_{1}=r \Gamma_{2}-q \Gamma_{3} \\
2 \dot{q} & =-p r-c \Gamma_{3} \quad \dot{\Gamma}_{2}=p \Gamma_{3}-r \Gamma_{1} \\
\dot{r} & =c \Gamma_{2} \quad \dot{\Gamma}_{3}=q \Gamma_{1}-p \Gamma_{2} .
\end{aligned}
$$

The system (3.4) has three well known first integrals of motion and a fourth first integral discovered by Kowalevski

$$
\begin{gathered}
2\left(p^{2}+q^{2}\right)+r^{2}=2 c \Gamma_{1}+6 l_{1} \\
2\left(p \Gamma_{1}+q \Gamma_{2}\right)+r \Gamma_{3}=2 l \\
\Gamma_{1}^{2}+\Gamma_{2}^{2}+\Gamma_{3}^{2}=1 \\
\left((p+i q)^{2}+\Gamma_{1}+i \Gamma_{2}\right)\left((p-i q)^{2}+\Gamma_{1}-i \Gamma_{2}\right)=k^{2} .
\end{gathered}
$$

After the change of variables

$$
\begin{array}{ll}
x_{1}=p+i q, & e_{1}=x_{1}^{2}+c\left(\Gamma_{1}+i \Gamma_{2}\right) \\
x_{2}=p-i q, & e_{2}=x_{2}^{2}+c\left(\Gamma_{1}-i \Gamma_{2}\right)
\end{array}
$$

the first integrals (3.5) transform into

$$
\begin{aligned}
r^{2} & =E+e_{1}+e_{2} \\
r c \Gamma_{3} & =F-x_{2} e_{1}-x_{1} e_{2} \\
c^{2} \Gamma_{3}^{2} & =G+x_{2}^{2} e_{1}+x_{1}^{2} e_{2} \\
e_{1} e_{2} & =k^{2},
\end{aligned}
$$


with $E=6 l_{1}-\left(x_{1}+x_{2}\right)^{2}, F=2 c l+x_{1} x_{2}\left(x_{1}+x_{2}\right), G=c^{2}-k^{2}-x_{1}^{2} x_{2}^{2}$. From the first integrals, one gets

$$
\left(E+e_{1}+e_{2}\right)\left(F+x_{2}^{2} e_{1}+x_{1}^{2} e_{2}\right)-\left(G-x_{2} e_{1}-x_{1} e_{2}\right)^{2}=0
$$

which can be rewritten in the form

$$
e_{1} P\left(x_{2}\right)+e_{2} P\left(x_{1}\right)+R_{1}\left(x_{1}, x_{2}\right)+k^{2}\left(x_{1}-x_{2}\right)^{2}=0
$$

where the polynomial $P$ is

$$
P\left(x_{i}\right)=x_{i}^{2} E+2 x_{1} F+G=-x_{i}^{4}+6 l_{1} x_{i}^{2}+4 l c x_{i}+c^{2}-k^{2}, i=1,2
$$

and

$$
\begin{aligned}
R_{1}\left(x_{1}, x_{2}\right)= & E G-F^{2}=-6 l_{1} x_{1}^{2} x_{2}^{2}-\left(c^{2}-k^{2}\right)\left(x_{1}+x_{2}\right)^{2} \\
& -4 l c\left(x_{1}+x_{2}\right) x_{1} x_{2}+6 l_{1}\left(c^{2}-k^{2}\right)-4 l^{2} c^{2} .
\end{aligned}
$$

Note that $P$ from the formula above depends only on one variable, which is not obvious from its definition. Denote

$$
R\left(x_{1}, x_{2}\right)=E x_{1} x_{2}+F\left(x_{1}+x_{2}\right)+G
$$

From (3.8), Kowalevski gets

$$
\left(\sqrt{P\left(x_{1}\right) e_{2}} \pm \sqrt{P\left(x_{2}\right) e_{1}}\right)^{2}=-\left(x_{1}-x_{2}\right)^{2} k^{2} \pm 2 k \sqrt{P\left(x_{1}\right) P\left(x_{2}\right)}-R_{1}\left(x_{1}, x_{2}\right) .
$$

After a few transformations, (3.9) can be written in the form

$$
\left[\sqrt{e_{1}} \frac{\sqrt{P\left(x_{2}\right)}}{x_{1}-x_{2}} \pm \sqrt{e_{2}} \frac{\sqrt{P\left(x_{1}\right)}}{x_{1}-x_{2}}\right]^{2}=\left(w_{1} \pm k\right)\left(w_{2} \mp k\right)
$$

where $w_{1}, w_{2}$ are the solutions of an equation, quadratic in $s$ :

$$
Q\left(s, x_{1}, x_{2}\right)=\left(x_{1}-x_{2}\right)^{2} s^{2}-2 R\left(x_{1}, x_{2}\right) s-R_{1}\left(x_{1}, x_{2}\right)=0 .
$$

The quadratic Eq. (3.11) is known as the Kowalevski fundamental equation. The discriminant separability condition for $Q\left(s, x_{1}, x_{2}\right)$ is satisfied

$$
\begin{gathered}
\mathcal{D}_{s}(Q)\left(x_{1}, x_{2}\right)=4 P\left(x_{1}\right) P\left(x_{2}\right) \\
\mathcal{D}_{x_{1}}(Q)\left(s, x_{2}\right)=-8 J(s) P\left(x_{2}\right), \mathcal{D}_{x_{2}}(Q)\left(s, x_{1}\right)=-8 J(s) P\left(x_{1}\right)
\end{gathered}
$$


with

$$
J(s)=s^{3}+3 l_{1} s^{2}+s\left(c^{2}-k^{2}\right)+3 l_{1}\left(c^{2}-k^{2}\right)-2 l^{2} c^{2} .
$$

The equations of motion (3.4) can be rewritten in new variables $\left(x_{1}, x_{2}, e_{1}, e_{2}, r, \Gamma_{3}\right)$ in the form:

$$
\begin{aligned}
& 2 \dot{x}_{1}=-i f_{1}, \quad \dot{e}_{1}=-m e_{1} \\
& 2 \dot{x}_{2}=i f_{2}, \quad \dot{e}_{2}=m e_{2} .
\end{aligned}
$$

There are two additional differential equations for $\dot{r}$ and $\dot{\Gamma}_{3}$. Here $m=i r$ and $f_{1}=$ $r x_{1}+c \Gamma_{3}, f_{2}=r x_{2}+c \Gamma_{3}$. One can easily check that

$$
f_{1}^{2}=P\left(x_{1}\right)+e_{1}\left(x_{1}-x_{2}\right)^{2}, \quad f_{2}^{2}=P\left(x_{2}\right)+e_{2}\left(x_{1}-x_{2}\right)^{2} .
$$

Further integration procedure is described in Kowalevski (1889), and in Dragović and Kukić (2014a).

We get the following

Theorem 3.2 (Dragović 2010) The Kowalevski fundamental equation represents a point pencil of conics given by their tangential equations

$$
\begin{aligned}
\hat{C}_{1}:-2 w_{1}^{2}+3 l_{1} w_{2}^{2}+2\left(c^{2}-k^{2}\right) w_{3}^{2}-4 c l w_{2} w_{3} & =0 \\
C_{2}: w_{2}^{2}-4 w_{1} w_{3} & =0 .
\end{aligned}
$$

The Kowalevski variables $w, x_{1}, x_{2}$ in this geometric settings are the pencil parameter, and the Darboux coordinates with respect to the conic $C_{2}$ respectively.

The Kowalevski case corresponds to the general case under the restrictions $a_{1}=$ $0 \quad a_{5}=0 \quad a_{0}=-2$. The last of these three relations is just normalization condition, provided $a_{0} \neq 0$. The Kowalevski parameters $l_{1}, l, c$ are calculated by the formulae

$$
l_{1}=\frac{a_{2}}{3} \quad l= \pm \frac{1}{2} \sqrt{-a_{4}+\sqrt{a_{4}+4 a_{3}^{2}}} \quad c=\mp \frac{a_{3}}{\sqrt{-a_{4}+\sqrt{a_{4}+4 a_{3}^{2}}}}
$$

provided that $l$ and $c$ are requested to be real.

Let us mention that Kowalevski in (1889), instead the relation (3.11), used the equivalent one, where the equivalence is obtained by putting $w=2 s-l_{1}$.

The Kowalevski change of variables is the following consequence of the discriminant separability property of the polynomial $F=Q$ :

$$
\begin{aligned}
& \frac{d x_{1}}{\sqrt{P\left(x_{1}\right)}}+\frac{d x_{2}}{\sqrt{P\left(x_{2}\right)}}=\frac{d w_{1}}{\sqrt{J\left(w_{1}\right)}} \\
& \frac{d x_{1}}{\sqrt{P\left(x_{1}\right)}}-\frac{d x_{2}}{\sqrt{P\left(x_{2}\right)}}=\frac{d w_{2}}{\sqrt{J\left(w_{2}\right)}} .
\end{aligned}
$$


The Kowalevski change of variables (see Eq. (3.15)) is infinitesimal of the correspondence which maps a pair of points $\left(M_{1}, M_{2}\right)$ to a pair of points $\left(S_{1}, S_{2}\right)$. Both pairs belong to a $\mathbb{P}^{1}$ as a factor of the appropriate elliptic curve. In our approach, there is a geometric view to this mapping as the correspondence which maps two tangents to the conic $C$ to the pair of conics from the pencil which contain the intersection point of the two lines.

If we apply fractional-linear transformations to transform the curve $\tilde{\Gamma}_{1}$ into the curve $\tilde{\Gamma}_{2}$, then the above correspondence is nothing else then the two-valued group operation $*_{c}$ on $\left(\tilde{\Gamma}_{2}, \mathbb{Z}_{2}\right)$.

Theorem 3.3 The Kowalevski change of variables is equivalent to infinitesimal of the action of the two valued coset group $\left(\tilde{\Gamma}_{2}, \mathbb{Z}_{2}\right)$ on $\mathbb{P}^{1}$ as a factor of the elliptic curve. Up to the fractional-linear transformation, it is equivalent to the operation of the two valued group $\left(\tilde{\Gamma}_{2}, \mathbb{Z}_{2}\right)$.

Now, the Kötter trick (see Kotter 1893; Dragović 2010) can be applied to the following commutative diagram.

Proposition 3.1 (Dragović 2010) The Kowalevski integration procedure may be codded in the following commutative diagram:

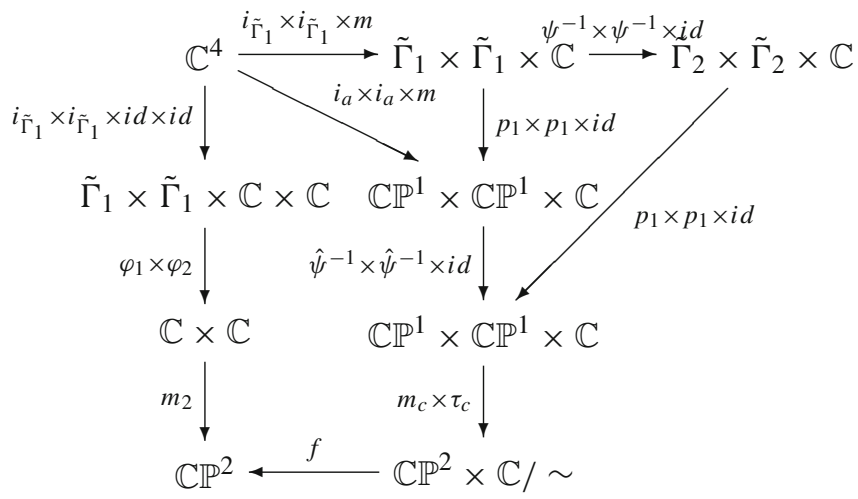

The mappings are defined as follows

$$
\begin{aligned}
& i_{\tilde{\Gamma}_{1}}: x \mapsto(x, \sqrt{P(x)}) \\
& m:(x, y) \mapsto x \cdot y \\
& i_{a}: x \mapsto(x, 1) \\
& p_{1}:(x, y) \mapsto x \\
& m_{c}:(x, y) \mapsto x *_{c} y \\
& \tau_{c}: x \mapsto(\sqrt{x},-\sqrt{x}) \\
& \varphi_{1}:\left(x_{1}, x_{2}, e_{1}, e_{2}\right) \mapsto \sqrt{e_{1}} \frac{\sqrt{P\left(x_{2}\right)}}{x_{1}-x_{2}} \\
& \varphi_{2}:\left(x_{1}, x_{2}, e_{1}, e_{2}\right) \mapsto \sqrt{e_{2}} \frac{\sqrt{P\left(x_{1}\right)}}{x_{1}-x_{2}} \\
& f:\left(\left(s_{1}, s_{2}, 1\right),(k,-k)\right) \mapsto\left[\left(\gamma^{-1}\left(s_{1}\right)+k\right)\left(\gamma^{-1}\left(s_{2}\right)-k\right),\left(\gamma^{-1}\left(s_{2}\right)+k\right)\left(\gamma^{-1}\left(s_{1}\right)-k\right)\right]
\end{aligned}
$$


From the Proposition 3.1 we see that the two-valued group plays an important role in the Kowalevski system and its generalizations.

\subsection{Systems of the Kowalevski Type: Definition}

Following Dragović and Kukić (2011, 2014a, b), we are going to present a class of dynamical systems, which generalizes the Kowalevski top. Instead of the Kowalevski fundamental equation (see formula (3.11)), the starting point here is an arbitrary discriminantly separable polynomial of degree two in each of three variables.

Given a discriminantly separable polynomial of the second degree in each of three variables

$$
\mathcal{F}\left(x_{1}, x_{2}, s\right):=A\left(x_{1}, x_{2}\right) s^{2}+B\left(x_{1}, x_{2}\right) s+C\left(x_{1}, x_{2}\right),
$$

such that

$$
\mathcal{D}_{s}(\mathcal{F})\left(x_{1}, x_{2}\right)=B^{2}-4 A C=4 P\left(x_{1}\right) P\left(x_{2}\right)
$$

and

$$
\begin{aligned}
& \mathcal{D}_{x_{1}}(\mathcal{F})\left(s, x_{2}\right)=4 P\left(x_{2}\right) J(s) \\
& \mathcal{D}_{x_{2}}(\mathcal{F})\left(s, x_{1}\right)=4 P\left(x_{1}\right) J(s) .
\end{aligned}
$$

Suppose, that a given system in variables $x_{1}, x_{2}, e_{1}, e_{2}, r, \gamma_{3}$, after some transformations reduces to

$$
\begin{aligned}
& 2 \dot{x}_{1}=-i f_{1}, \quad \dot{e}_{1}=-m e_{1}, \\
& 2 \dot{x}_{2}=i f_{2}, \quad \dot{e}_{2}=m e_{2},
\end{aligned}
$$

where

$$
f_{1}^{2}=P\left(x_{1}\right)+e_{1} A\left(x_{1}, x_{2}\right), \quad f_{2}^{2}=P\left(x_{2}\right)+e_{2} A\left(x_{1}, x_{2}\right) .
$$

Suppose additionally, that the first integrals of the initial system reduce to a relation

$$
P\left(x_{2}\right) e_{1}+P\left(x_{1}\right) e_{2}=C\left(x_{1}, x_{2}\right)-e_{1} e_{2} A\left(x_{1}, x_{2}\right) .
$$

The equations for $\dot{r}$ and $\dot{\Gamma}_{3}$ are not specified for the moment and $m$ is a function of system's variables.

If a system satisfies the above assumptions we will call it a system of the Kowalevski type. As it has been pointed out in the previous subsection, see formulae $(3.8,3.11$, $3.12,3.13)$, the Kowalevski top is an example of the systems of the Kowalevski type.

The following theorem is quite general, and concerns all the systems of the Kowalevski type. It explains in full a subtle mechanism of a quite miraculous jump in genus, from one to two, in integration procedure, which has been observed in the Kowalevski top, and now it is going to be established as a characteristic property of the whole new class of systems. 
Theorem 3.4 Given a system which reduces to $(3.17,3.18,3.19)$. Then the system is linearized on the Jacobian of the curve

$$
y^{2}=J(z)(z-k)(z+k)
$$

where $J$ is a polynomial factor of the discriminant of $\mathcal{F}$ as a polynomial in $x_{1}$ and $k$ is a constant such that

$$
e_{1} e_{2}=k^{2}
$$

The last Theorem basically formalizes the original considerations of Kowalevski, in a slightly more general context of the discriminantly separable polynomials. A proof is presented in Dragović and Kukić (2014b).

In the following subsections we present the Sokolov system given in Sokolov (2002) as an example of systems of the Kowalevski type, and one more recent example of the systems of the Kowalevski type.

\subsection{An Example of Systems of the Kowalevski Type}

Consider the Hamiltonian (see Sokolov 2002; Sokolov and Tsiganov 2001)

$$
\hat{H}=M_{1}^{2}+M_{2}^{2}+2 M_{3}^{2}+2 c_{1} \gamma_{1}+2 c_{2}\left(\gamma_{2} M_{3}-\gamma_{3} M_{2}\right)
$$

on $e(3)$ with Lie-Poisson brackets

$$
\left\{M_{i}, M_{j}\right\}=\epsilon_{i j k} M_{k}, \quad\left\{M_{i}, \gamma_{j}\right\}=\epsilon_{i j k} \gamma_{k}, \quad\left\{\gamma_{i}, \gamma_{j}\right\}=0
$$

where $\epsilon_{i j k}$ is the totally skew-symetric tensor. In Komarov et al. (2003), an explicit mapping of the integrable system on $e$ (3) with Hamiltonian (3.20) and the Kowalevski top on $s o(3,1)$ has been found and a separation of variables for the system (3.20) was performed. In this section we will show that the system fits into the class of the systems of the Kowalevski type.

The Lie-Poisson brackets (3.21) have two well known Casimir functions

$$
\begin{aligned}
& \gamma_{1}^{2}+\gamma_{2}^{2}+\gamma_{3}^{2}=a, \\
& \gamma_{1} M_{1}+\gamma_{2} M_{2}+\gamma_{3} M 3=b .
\end{aligned}
$$

Following Komarov et al. (2003) and Kowalevski (1889) we introduce new variables

$$
z_{1}=M_{1}+i M_{2}, \quad z_{2}=M_{1}-i M_{2}
$$

and

$$
\begin{aligned}
& e_{1}=z_{1}^{2}-2 c_{1}\left(\gamma_{1}+i \gamma_{2}\right)-c_{2}^{2} a-c_{2}\left(2 \gamma_{2} M_{3}-2 \gamma_{3} M_{2}+2 i\left(\gamma_{3} M_{1}-\gamma_{1} M_{3}\right)\right) \\
& e_{2}=z_{2}^{2}-2 c_{1}\left(\gamma_{1}-i \gamma_{2}\right)-c_{2}^{2} a-c_{2}\left(2 \gamma_{2} M_{3}-2 \gamma_{3} M_{2}+2 i\left(\gamma_{1} M_{3}-\gamma_{3} M_{1}\right)\right) .
\end{aligned}
$$


The second integral of motion for system (3.20) then may be written as

$$
e_{1} e_{2}=k^{2} \text {. }
$$

The equations of motion for new variables $z_{i}, e_{i}$ can be written in the form of (3.17) and (3.18), as we supposed in definition of Kowalevski type systems. It is easy to prove that:

$$
\dot{e}_{1}=-4 i M_{3} e_{1}, \quad \dot{e}_{2}=4 i M_{3} e_{2}
$$

and

$$
\begin{aligned}
& -{\dot{z_{1}}}^{2}=P\left(z_{1}\right)+e_{1}\left(z_{1}-z_{2}\right)^{2}, \\
& -\dot{z}_{2}^{2}=P\left(z_{2}\right)+e_{2}\left(z_{1}-z_{2}\right)^{2}
\end{aligned}
$$

where $\mathrm{P}$ is the fourth degree polynomial given by

$$
P(z)=-z^{4}+2 H z^{2}-8 c_{1} b z-k^{2}+4 a c_{1}^{2}-2 c_{2}^{2}\left(2 b^{2}-H a\right)+c_{2}^{4} a .
$$

In Komarov et al. (2003) the biquadratic form and the separated variables are defined as the next step:

$$
\begin{aligned}
F\left(z_{1}, z_{2}\right) & =-\frac{1}{2}\left(P\left(z_{1}\right)+P\left(z_{2}\right)+\left(z_{1}^{2}-z_{2}^{2}\right)^{2}\right), \\
s_{1,2} & =\frac{F\left(z_{1}, z_{2}\right) \pm \sqrt{P\left(z_{1}\right) P\left(z_{2}\right)}}{2\left(z_{1}-z_{2}\right)^{2}}
\end{aligned}
$$

such that

$$
\dot{s_{1}}=\frac{\sqrt{P_{5}\left(s_{1}\right)}}{s_{1}-s_{2}}, \quad \dot{s_{2}}=\frac{\sqrt{P_{5}\left(s_{2}\right)}}{s_{2}-s_{1}}, \quad P_{5}(s)=P_{3}(s) P_{2}(s)
$$

with

$$
\begin{aligned}
& P_{3}(s)=s\left(4 s^{2}+4 s H+H^{2}-k^{2}+4 c_{1}^{2} a+2 c_{2}^{2}\left(H a-2 b^{2}\right)+c_{2}^{4} a^{2}\right)+4 c_{1}^{2} b^{2} \\
& P_{2}(s)=4 s^{2}+4\left(H+c_{2}^{2} a\right) s+H^{2}-k^{2}+2 c_{2}^{2} h a+c_{2}^{4} a^{2} .
\end{aligned}
$$

To fit this system into the class of the Kowalevski type systems, we still need to show that a relation of the form of (3.19) is satisfied and to relate it with a corresponding discriminantly separable polynomial in the form of (3.16). Starting from the equations

$$
\dot{z_{1}}=-2 M_{3}\left(M_{1}-i M_{2}\right)+2 c_{2}\left(\gamma_{1} M_{2}-\gamma_{2} M_{1}\right)+2 c_{1} \gamma_{3}
$$

and

$$
\dot{z_{2}}=-2 M_{3}\left(M_{1}+i M_{2}\right)+2 c_{2}\left(\gamma_{1} M_{2}-\gamma_{2} M_{1}\right)+2 c_{1} \gamma_{3}
$$


one can prove that

$$
\dot{z_{1}} \cdot \dot{z_{2}}=-\left(F\left(z_{1}, z_{2}\right)+\left(H+c_{2}^{2} a\left(z_{1}-z_{2}\right)^{2}\right)\right)
$$

where $F\left(z_{1}, z_{2}\right)$ is given by (3.25). After equating the square of $\dot{z_{1}} \dot{z_{2}}$ from previous relation and $\dot{z}_{1}^{2} \cdot \dot{z}_{2}^{2}$ with $\dot{z}_{i}^{2}$ given by (3.23) we get

$$
\begin{aligned}
& \left(z_{1}-z_{2}\right)^{2}\left[2 F\left(z_{1}, z_{2}\right)\left(H+c_{2}^{2} a\right)+\left(z_{1}-z_{2}^{2}\right)^{4}\left(H+c_{2}^{2} a\right)^{2}-P\left(z_{1}\right) e_{2}-P\left(z_{2}\right) e_{1}\right. \\
& \left.-e_{1} e_{2}\left(z_{1}-z_{2}\right)^{2}\right]+F^{2}\left(z_{1}, z_{2}\right)-P\left(z_{1}\right) P\left(z_{2}\right)=0
\end{aligned}
$$

Denote with $C\left(z_{1}, z_{2}\right)$ biquadratic polynomial such that

$$
F^{2}\left(z_{1}, z_{2}\right)-P\left(z_{1}\right) P\left(z_{2}\right)=\left(z_{1}-z_{2}\right)^{2} C\left(z_{1}, z_{2}\right)
$$

Then we can rewrite relation (3.26) in the form of (3.19):

$$
P\left(z_{1}\right) e_{2}+P\left(z_{2}\right) e_{1}=\tilde{C}\left(z_{1}, z_{2}\right)-e_{1} e_{2}\left(z_{1}-z_{2}\right)^{2}
$$

with

$$
\tilde{C}\left(z_{1}, z_{2}\right)=C\left(z_{1}, z_{2}\right)+2 F\left(z_{1}, z_{2}\right)\left(H+c_{2}^{2} a\right)+\left(H+c_{2}^{2} a\right)^{2}\left(z_{1}-z_{2}\right)^{2} .
$$

Further integration procedure may be done following Theorem 3.4, since all assumptions on the systems of the Kowalevski type are satisfied with (3.26), (3.27) and (3.23). A discriminantly separable polynomial of three variables degree two in each which "plays role" of the Kowalevski fundamental equation in this case is

$$
\tilde{F}\left(z_{1}, z_{2}, s\right)=\left(z_{1}-z_{2}\right)^{2} s^{2}+\tilde{B}\left(z_{1}, z_{2}\right) s+\tilde{C}\left(z_{1}, z_{2}\right)
$$

with

$$
\tilde{B}\left(z_{1}, z_{2}\right)=F\left(z_{1}, z_{2}\right)+\left(H+c_{2}^{2} a\right)\left(z_{1}-z_{2}\right)^{2} .
$$

Discriminants of (3.29) as a polynomial in $s$ and in $z_{i}$, for $i=1,2$ are

$$
\begin{aligned}
& \mathcal{D}_{s}(\tilde{F})\left(z_{1}, z_{2}\right)=P\left(z_{1}\right) P\left(z_{2}\right) \\
& \mathcal{D}_{z_{1}}(\tilde{F})\left(s, z_{2}\right)=8 J(s) P\left(z_{2}\right), \mathcal{D}_{z_{2}}(Q)\left(s, z_{1}\right)=8 J(s) P\left(z_{1}\right)
\end{aligned}
$$

where $J$ is polynomial of the third degree

$$
\begin{aligned}
J= & s^{3}+\left(H+3 a c_{2}^{2}\right) s^{2}+\left(4 c_{2}^{2} H a+4 c_{2}^{4} a^{2}+4 a c_{1}^{2}-4 c_{2}^{2} b^{2}-k^{2}\right) s-8 c_{1}^{2} b^{2} \\
& -4 c_{2}^{4} a b^{2}+4 c_{1}^{2} a^{2} c_{2}^{2}-k^{2} c_{2}^{2} a-H k^{2}+2 a H^{2} c_{2}^{2}-4 H b^{2} c_{2}^{2}+4 H c_{1}^{2} a \\
& +4 c_{2}^{4} H a^{2}+2 c_{2}^{6} a^{3} .
\end{aligned}
$$


The roots of (3.29) are related with $s_{i}$ from Komarov et al. (2003) in the following manner:

$$
\tilde{s_{i}}=s_{i}+\frac{H+c_{2}^{2} a}{2} \text {. }
$$

Finally, as a result of direct application of Theorem 3.4 we get

$$
\begin{aligned}
& \frac{d \tilde{s_{1}}}{\sqrt{\Phi\left(\tilde{s_{1}}\right)}}+\frac{d \tilde{s_{2}}}{\sqrt{\Phi\left(\tilde{s_{2}}\right)}}=0 \\
& \frac{\tilde{s_{1}} d \tilde{s_{1}}}{\sqrt{\Phi\left(\tilde{s_{1}}\right)}}+\frac{\tilde{s_{2}} d \tilde{s_{2}}}{\sqrt{\Phi\left(\tilde{s_{2}}\right)}}=d t
\end{aligned}
$$

where

$$
\Phi(s)=-4 J(s)(s-k)(s+k) .
$$

\subsection{Another Example of an Integrable System of the Kowalevski Type}

Now, we are going to present one more example of a system of the Kowalevski type. Let us consider the next system of differential equations:

$$
\begin{aligned}
\dot{p} & =-r q \\
\dot{q} & =-r p-\gamma_{3} \\
\dot{r} & =-2 q(2 p+1)-2 \gamma_{2} \\
\dot{\gamma}_{1} & =2\left(q \gamma_{3}-r \gamma_{2}\right) \\
\dot{\gamma}_{2} & =2\left(p \gamma_{3}-r \gamma_{1}\right) \\
\dot{\gamma}_{3} & =2\left(p^{2}-q^{2}\right) q-2 q \gamma_{1}+2 p \gamma_{2} .
\end{aligned}
$$

Lemma 3.2 The system (3.30) preserves the standard measure.

After a change of variables

$$
\begin{array}{ll}
x_{1}=p+q, & e_{1}=x_{1}^{2}+\gamma_{1}+\gamma_{2}, \\
x_{2}=p-q, & e_{2}=x_{2}^{2}+\gamma_{1}-\gamma_{2},
\end{array}
$$


the system (3.30) becomes

$$
\begin{aligned}
\dot{x}_{1} & =-r x_{1}-\gamma_{3} \\
\dot{x}_{2} & =r x_{2}+\gamma_{3} \\
\dot{e}_{1} & =-2 r e_{1} \\
\dot{e}_{2} & =2 r e_{2} \\
\dot{r} & =-x_{1}+x_{2}-e_{1}+e_{2} \\
\dot{\gamma_{3}} & =x_{2} e_{1}-x_{1} e_{2} .
\end{aligned}
$$

The first integrals of the system (3.31) can be presented in the form

$$
\begin{aligned}
r^{2} & =2\left(x_{1}+x_{2}\right)+e_{1}+e_{2}+h \\
r \gamma_{3} & =-x_{1} x_{2}-x_{2} e_{1}-x_{1} e_{2}-\frac{g_{2}}{4} \\
\gamma_{3}^{2} & =x_{2}^{2} e_{1}+x_{1}^{2} e_{2}-\frac{g_{3}}{2} \\
e_{1} \cdot e_{2} & =k^{2} .
\end{aligned}
$$

From the integrals (3.32) we get a relation of the form (3.19)

$$
\begin{aligned}
& \left(x_{1}-x_{2}\right)^{2} e_{1} e_{2}+\left(2 x_{1}^{3}+h x_{1}^{2}-\frac{g_{2}}{2} x_{1}-\frac{g_{3}}{2}\right) e_{2}+\left(2 x_{2}^{3}+h x_{2}^{2}-\frac{g_{2}}{2} x_{2}-\frac{g_{3}}{2}\right) e_{1} \\
& -\left(x_{1}^{2} x_{2}^{2}+x_{1} x_{2} \frac{g_{2}}{2}+g_{3}\left(x_{1}+x_{2}+\frac{h}{2}\right)+\frac{g_{2}^{2}}{16}\right)=0 .
\end{aligned}
$$

Without loss of generality, we can assume $h=0$ (this can be achieved by a simple linear change of variables $\left.x_{i} \mapsto x_{i}-h / 6, \quad s \mapsto s-h / 6\right)$, thus we can use directly the Weierstrass $\wp$ function. Following the procedure described in Theorem 3.4 we get

$$
\begin{aligned}
& \frac{d x_{1}}{\sqrt{P\left(x_{1}\right)}}+\frac{d x_{2}}{\sqrt{P\left(x_{2}\right)}}=\frac{d s_{1}}{\sqrt{P\left(s_{1}\right)}} \\
& \frac{d x_{1}}{\sqrt{P\left(x_{1}\right)}}-\frac{d x_{2}}{\sqrt{P\left(x_{2}\right)}}=\frac{d s_{2}}{\sqrt{P\left(s_{2}\right)}}
\end{aligned}
$$

where $P(x)$ denotes the polynomial

$$
P(x)=2 x^{3}-\frac{g_{2}}{2} x-\frac{g_{3}}{2},
$$


and $s_{1}, s_{2}$ are the solutions of quadratic equation in $s$ :

$$
\begin{aligned}
\mathcal{F}\left(x_{1}, x_{2}, s\right) & :=A\left(x_{1}, x_{2}\right) s^{2}+B\left(x_{1}, x_{2}\right) s+C\left(x_{1}, x_{2}\right) \\
= & \left(x_{1}-x_{2}\right)^{2} s^{2}+\left(-2 x_{1} x_{2}\left(x_{1}+x_{2}\right)+\frac{g_{2}}{2}\left(x_{1}+x_{2}\right)+g_{3}\right) s \\
& +x_{1}^{2} x_{2}^{2}+x_{1} x_{2} \frac{g_{2}}{2}+g_{3}\left(x_{1}+x_{2}\right)+\frac{g_{2}^{2}}{16}=0 .
\end{aligned}
$$

Finally, we get

Corollary 3.1 The system of differential Eq. (3.30) is integrated through the solutions of the system

$$
\begin{aligned}
& \frac{d s_{1}}{\sqrt{\Phi\left(s_{1}\right)}}+\frac{d s_{2}}{\sqrt{\Phi\left(s_{2}\right)}}=0 \\
& \frac{s_{1} d s_{1}}{\sqrt{\Phi\left(s_{1}\right)}}+\frac{s_{2} d s_{2}}{\sqrt{\Phi\left(s_{2}\right)}}=2 d t
\end{aligned}
$$

where $\Phi(s)=P(s)(s-k)(s+k)$.

\subsection{Another Class of Systems of the Kowalevski Type}

In this section we will consider another class of systems of Kowalevski type. We consider a situation analogue to that from the beginning of the Sect. 3.5. The only difference is that the systems we are going to consider now, reduce to (3.17), where

$$
\begin{aligned}
& f_{1}^{2}=P\left(x_{1}\right)-\frac{C}{e_{2}} \\
& f_{2}^{2}=P\left(x_{2}\right)-\frac{C}{e_{1}} .
\end{aligned}
$$

The next Proposition is an analogue of Theorem 3.4. Thus, the new class of systems also has a striking property of jumping genus in integration procedure.

Proposition 3.2 Given a system which reduces to (3.17), where

$$
\begin{aligned}
& f_{1}^{2}=P\left(x_{1}\right)-\frac{C}{e_{2}} \\
& f_{2}^{2}=P\left(x_{2}\right)-\frac{C}{e_{1}}
\end{aligned}
$$

and integrals reduce to (3.19); A, C, P form a discriminantly separable polynomial $\mathcal{F}$ given with (3.16). Then the system is linearized on the Jacobian of the curve

$$
y^{2}=J(z)(z-k)(z+k)
$$


where $J$ is a polynomial factor of the discriminant of $\mathcal{F}$ as a polynomial in $x_{1}$ and $k$ is a constant such that

$$
e_{1} e_{2}=k^{2}
$$

Proof Although the proof is a variation of the proof of the Theorem 3.4 there are some interesting steps and algebraic transformations we point out in next few lines. In the same manner as in Theorem 3.4 we obtain

$$
\begin{aligned}
& \left(\sqrt{e_{1}} \sqrt{\frac{P\left(x_{2}\right)}{A}}+\sqrt{e_{2}} \sqrt{\frac{P\left(x_{1}\right)}{A}}\right)^{2}=\left(s_{1}+k\right)\left(s_{2}-k\right) \\
& \left(\sqrt{e_{1}} \sqrt{\frac{P\left(x_{2}\right)}{A}}-\sqrt{e_{2}} \sqrt{\frac{P\left(x_{1}\right)}{A}}\right)^{2}=\left(s_{1}-k\right)\left(s_{2}+k\right)
\end{aligned}
$$

where $s_{1}, s_{2}$ are the solutions of the quadratic equation

$$
\mathcal{F}\left(x_{1}, x_{2}, s\right)=0
$$

in $s$. From the last equations, dividing with $k=\sqrt{e_{1} e_{2}}$ we get

$$
\begin{aligned}
& 2 \sqrt{\frac{P\left(x_{2}\right)}{e_{2} A}}=\frac{1}{k}\left(\sqrt{\left(s_{1}+k\right)\left(s_{2}-k\right)}+\sqrt{\left(s_{1}-k\right)\left(s_{2}+k\right)}\right) \\
& 2 \sqrt{\frac{P\left(x_{1}\right)}{e_{1} A}}=\frac{1}{k}\left(\sqrt{\left(s_{1}+k\right)\left(s_{2}-k\right)}-\sqrt{\left(s_{1}-k\right)\left(s_{2}+k\right)}\right) .
\end{aligned}
$$

Using $\left(s_{1}-s_{2}\right)^{2}=4 \frac{P\left(x_{1}\right) P\left(x_{2}\right)}{A^{2}}$, we get

$$
\begin{aligned}
f_{1}^{2} & =P\left(x_{1}\right)-\frac{C\left(x_{1}, x_{2}\right)}{e_{2}}=\frac{\left(s_{1}-s_{2}\right)^{2} A^{2}}{4 P\left(x_{2}\right)}-\frac{C}{e_{2}}=\frac{A^{2}}{4 P\left(x_{2}\right)}\left[\left(s_{1}-s_{2}\right)^{2}-\frac{C}{A} \frac{4 P\left(x_{2}\right)}{e_{2} A}\right] \\
& =\frac{P\left(x_{1}\right)}{\left(s_{1}-s_{2}\right)^{2}}\left[\left(s_{1}-s_{2}\right)^{2}-s_{1} s_{2} \frac{1}{k^{2}}\left(\sqrt{\left(s_{1}+k\right)\left(s_{2}-k\right)}+\sqrt{\left(s_{1}-k\right)\left(s_{2}+k\right)}\right)^{2}\right] \\
& =\frac{P\left(x_{1}\right)}{\left(s_{1}-s_{2}\right)^{2}}\left[s_{1}^{2}-2 s_{1} s_{2}+s_{2}^{2}-\frac{2 s_{1} s_{2}}{k^{2}}\left(s_{1} s_{2}-k^{2}+\sqrt{\left(s_{1}^{2}-k^{2}\right)\left(s_{2}^{2}-k^{2}\right)}\right)\right] \\
& =\frac{P\left(x_{1}\right)}{k^{2}\left(s_{1}-s_{2}\right)^{2}}\left[k^{2}\left(s_{1}^{2}+s_{2}^{2}\right)-2 s_{1}^{2} s_{2}^{2}-2 s_{1} s_{2} \sqrt{\left(s_{1}^{2}-k^{2}\right)\left(s_{2}^{2}-k^{2}\right)}\right] \\
& =-\frac{P\left(x_{1}\right)}{k^{2}\left(s_{1}-s_{2}\right)^{2}}\left[s_{2} \sqrt{s_{1}^{2}-k^{2}}+s_{1} \sqrt{s_{2}^{2}-k^{2}}\right]^{2} .
\end{aligned}
$$

Similarly

$$
f_{2}^{2}=-\frac{P\left(x_{2}\right)}{k^{2}\left(s_{1}-s_{2}\right)^{2}}\left[s_{2} \sqrt{s_{1}^{2}-k^{2}}-s_{1} \sqrt{s_{2}^{2}-k^{2}}\right]^{2} .
$$


From the last two equations and from the equations of motion, we get

$$
\begin{aligned}
& 2 \dot{x}_{1}=\frac{-l \sqrt{P\left(x_{1}\right)}}{k\left(s_{1}-s_{2}\right)}\left[s_{2} \sqrt{s_{1}^{2}-k^{2}}+s_{1} \sqrt{s_{2}^{2}-k^{2}}\right] \\
& 2 \dot{x}_{2}=\frac{-l \sqrt{P\left(x_{2}\right)}}{k\left(s_{1}-s_{2}\right)}\left[s_{2} \sqrt{s_{1}^{2}-k^{2}}-s_{1} \sqrt{s_{2}^{2}-k^{2}}\right],
\end{aligned}
$$

and

$$
\begin{aligned}
& \frac{d x_{1}}{\sqrt{P\left(x_{1}\right)}}+\frac{d x_{2}}{\sqrt{P\left(x_{2}\right)}}=\frac{-\imath s_{2} \sqrt{s_{1}^{2}-k^{2}}}{k\left(s_{1}-s_{2}\right)} d t \\
& \frac{d x_{1}}{\sqrt{P\left(x_{1}\right)}}-\frac{d x_{2}}{\sqrt{P\left(x_{2}\right)}}=\frac{-t s_{1} \sqrt{s_{2}^{2}-k^{2}}}{k\left(s_{1}-s_{2}\right)} d t .
\end{aligned}
$$

Discriminant separability condition (see Corollary 1 from Dragović 2010) gives

$$
\begin{aligned}
& \frac{d x_{1}}{\sqrt{P\left(x_{1}\right)}}+\frac{d x_{2}}{\sqrt{P\left(x_{2}\right)}}=\frac{d s_{1}}{\sqrt{J\left(s_{1}\right)}} \\
& \frac{d x_{1}}{\sqrt{P\left(x_{1}\right)}}-\frac{d x_{2}}{\sqrt{P\left(x_{2}\right)}}=-\frac{d s_{2}}{\sqrt{J\left(s_{2}\right)}} .
\end{aligned}
$$

Finally

$$
\begin{aligned}
& \frac{d s_{1}}{\sqrt{\Phi\left(s_{1}\right)}}+\frac{d s_{2}}{\sqrt{\Phi\left(s_{2}\right)}}=\frac{l}{k} d t \\
& \frac{s_{1} d s_{1}}{\sqrt{\Phi\left(s_{1}\right)}}+\frac{s_{2} d s_{2}}{\sqrt{\Phi\left(s_{2}\right)}}=0,
\end{aligned}
$$

where

$$
\Phi(s)=J(s)(s-k)(s+k)
$$

is a polynomial of degree up to six.

\subsection{A Deformation of the Kowalevski Top}

In this Section we are going to derive the explicit solutions in genus two theta-functions of the Jurdjevic elasticae Jurdjevic (1999a) and for similar systems (Komarov 1981; Komarov and Kuznetsov 1990). First, we show that we can get the elasticae from the Kowalevski top by using the simplest gauge transformations of the discriminantly separable polynomials.

Consider a discriminantly separable polynomial

$$
\mathcal{F}\left(x_{1}, x_{2}, s\right):=s^{2} A+s B+C
$$


where

$$
A=\left(x_{1}-x_{2}\right)^{2}, \quad B=-2\left(E x_{1} x_{2}+F\left(x_{1}+x_{2}\right)+G\right), \quad C=F^{2}-E G .
$$

A simple affine gauge transformation $s \mapsto t+\alpha$ transforms $\mathcal{F}\left(x_{1}, x_{2}, s\right)$ into

$$
\mathcal{F}_{\alpha}\left(x_{1}, x_{2}, t\right)=t^{2} A_{\alpha}+t B_{\alpha}+C_{\alpha},
$$

with

$$
A_{\alpha}=A, B_{\alpha}=B+2 \alpha A, \quad C_{\alpha}=C+\alpha B+\alpha^{2} A .
$$

Next, we denote $F_{\alpha}=F+\alpha F_{1}, E_{\alpha}=E+\alpha E_{1}, G_{\alpha}=G+\alpha G_{1}$. From

$$
C_{\alpha}=F_{\alpha}^{2}-E_{\alpha} G_{\alpha},
$$

by equating powers of $\alpha$, we get

$$
B=2 F F_{1}-E_{1} G-E G_{1}, \quad A=F_{1}^{2}-E_{1} G_{1} .
$$

From (3.42) one obtains

$$
F_{1}=-\left(x_{1}+x_{2}\right), \quad G_{1}=2 x_{1} x_{2}, \quad E_{1}=2 .
$$

One easily checks that $F_{1}^{2}-E_{1} G_{1}=A$,

$$
\begin{aligned}
E_{\alpha} & =6 l_{1}-\left(x_{1}+x_{2}\right)^{2}+2 \alpha \\
F_{\alpha} & =2 c l+x_{1} x_{2}\left(x_{1}+x_{2}\right)-\alpha\left(x_{1}+x_{2}\right) \\
G_{\alpha} & =c^{2}-k^{2}-x_{1}^{2} x_{2}^{2}+2 \alpha x_{1} x_{2} .
\end{aligned}
$$

Not being aware on that time of the fundamental work of Komarov (1981) and Komarov and Kuznetsov (1990), where the following deformations of the Kowalevski case were constructed and considered, Jurdjevic associated these systems to the Kirchhoff elastic problem, see (Jurdjevic 1999a). The systems are defined by the Hamiltonians

$$
H=\frac{1}{4}\left(M_{1}^{2}+M_{2}^{2}+2 M_{3}^{2}\right)+\gamma_{1}
$$

where the deformed Poisson structures $\{\cdot, \cdot\}_{\tau}$ are defined by

$$
\left\{M_{i}, M_{j}\right\}_{\tau}=\epsilon_{i j k} M_{k}, \quad\left\{M_{i}, \gamma_{j}\right\}_{\tau}=\epsilon_{i j k} \gamma_{k}, \quad\left\{\gamma_{i}, \gamma_{j}\right\}_{\tau}=\tau \epsilon_{i j k} M_{k}
$$

and where the deformation parameter takes values $\tau=0,1,-1$. These structures correspond to $e(3), s o(4)$, and $s o(3,1)$ respectively. The classical Kowalevski case corresponds to the case $\tau=0$. These systems have been rediscovered by several 
authors in the meantime. Here, we are giving explicit formulae in theta-functions for the solutions of these systems.

Denote

$$
\begin{aligned}
& e_{1}=x_{1}^{2}-\left(\gamma_{1}+i \gamma_{2}\right)+\tau \\
& e_{2}=x_{2}^{2}-\left(\gamma_{1}-i \gamma_{2}\right)+\tau,
\end{aligned}
$$

where

$$
x_{1,2}=\frac{M_{1} \pm i M_{2}}{2} .
$$

The integrals of motion

$$
\begin{aligned}
& I_{1}=e_{1} e_{2} \\
& I_{2}=H \\
& I_{3}=\gamma_{1} M_{1}+\gamma_{2} M_{2}+\gamma_{3} M_{3} \\
& I_{4}=\gamma_{1}^{2}+\gamma_{2}^{2}+\gamma_{3}^{2}+\tau\left(M_{1}^{2}+M_{2}^{2}+M_{3}^{2}\right)
\end{aligned}
$$

may be rewritten in the form

$$
\begin{aligned}
k^{2} & =I_{1}=e_{1} \cdot e_{2} \\
M_{3}^{2} & =e_{1}+e_{2}+\hat{E}\left(x_{1}, x_{2}\right) \\
-M_{3} \gamma_{3} & =-x_{2} e_{1}-x_{1} e_{2}+\hat{F}\left(x_{1}, x_{2}\right) \\
\gamma_{3}^{2} & =x_{2}^{2} e_{1}+x_{1}^{2} e_{2}+\hat{G}\left(x_{1}, x_{2}\right),
\end{aligned}
$$

where

$$
\begin{aligned}
& \hat{G}\left(x_{1}, x_{2}\right)=-x_{1}^{2} x_{2}^{2}-2 \tau x_{1} x_{2}-2 \tau I_{2}+\tau^{2}+I_{4}-I_{1} \\
& \hat{F}\left(x_{1}, x_{2}\right)=\left(x_{1} x_{2}+\tau\right)\left(x_{1}+x_{2}\right)-I_{3} \\
& \hat{E}\left(x_{1}, x_{2}\right)=-\left(x_{1}+x_{2}\right)^{2}+2\left(I_{2}-\tau\right) .
\end{aligned}
$$

Lemma 3.3 Let $c=-1$. If

$$
\tau=-\alpha, I_{2}=3 l_{1}, I_{3}=2 l, I_{4}=1-\alpha^{2}-6 l_{1} \alpha,
$$

then the relations (3.47) and (3.46) coincide.

Let us point out that the previous consideration does not establish an isomorphism between the Kowalevski top and the Jurdjevic elastica. It does not provide a coordinate transformation which would map the former to the latter. Nevertheless, the previous Lemma opens a possibility to integrate the latter system along the same scheme used for the former system: the generalized Kötter trick is related to discriminatly separable 
polynomials, see Dragović (2010), and thus applicable to the Jurdjevic elasticae as well, see Dragović and Kukić (2014b).

More explicitly, we apply the generalized Kötter transformation derived in Dragović (2010) to obtain the expressions for $M_{i}, \gamma_{i}$ in terms of $P_{i}$ and $P_{i j}$-functions for $i, j=1,2,3$. A generalization of the Kötter transformation which provides commuting separated variables for the above systems was performed in Komarov and Kuznetsov (1990), Komarov et al. (2003). First, we rewrite the equations of motion for Jurdjevic elasticae:

$$
\begin{aligned}
& \dot{M}_{1}=\frac{M_{2} M_{3}}{2} \\
& \dot{M}_{2}=-\frac{M_{1} M_{3}}{2}+\gamma_{3} \\
& \dot{M}_{3}=-\gamma_{2} \\
& \dot{\gamma}_{1}=-\frac{M_{2} \gamma_{3}}{2}+M_{3} \gamma_{2} \\
& \dot{\gamma}_{2}=\frac{M_{1} \gamma_{3}}{2}-M_{3} \gamma_{1}+\tau M_{3} \\
& \dot{\gamma}_{3}=-\frac{M_{1} \gamma_{2}}{2}+\frac{M_{2} \gamma_{1}}{2}-\tau M_{2} .
\end{aligned}
$$

Now we introduce the following notation:

$$
\begin{aligned}
R\left(x_{1}, x_{2}\right) & =\hat{E} x_{1} x_{2}+\hat{F}\left(x_{1}+x_{2}\right)+\hat{G}, \\
R_{1}\left(x_{1}, x_{2}\right) & =\hat{E} \hat{G}-\hat{F}^{2} \\
P\left(x_{i}\right) & =\hat{E} x_{i}^{2}+2 \hat{F} x_{i}+\hat{G}, \quad i=1,2 .
\end{aligned}
$$

Lemma 3.4 For a polynomial $\mathcal{F}\left(x_{1}, x_{2}, s\right)$ given by

$$
\mathcal{F}\left(x_{1}, x_{2}, s\right)=\left(x_{1}-x_{2}\right)^{2} s^{2}-2 R\left(x_{1}, x_{2}\right) s-R_{1}\left(x_{1}, x_{2}\right),
$$

there exist polynomials $A\left(x_{1}, x_{2}, s\right), B\left(x_{1}, x_{2}, s\right), f(s), A_{0}(s)$ such that the following identity holds

$$
\mathcal{F}\left(x_{1}, x_{2}, s\right) A_{0}(s)=A^{2}\left(x_{1}, x_{2}, s\right)+f(s) B\left(x_{1}, x_{2}, s\right) .
$$

The polynomials are defined by the formulae:

$$
\begin{aligned}
A_{0}(s)= & 2 s+2 I_{1}-2 \tau \\
f(s)= & 2 s^{3}+2\left(I_{1}-3 \tau\right) s^{2}+\left(-4 \tau\left(I_{1}-\tau\right)-2 I_{2}+4 \tau^{2}+2 I_{4}-4 \tau I_{2}\right) s \\
& +\left(I_{1}-\tau\right)\left(-2 I_{1}+2 \tau^{2}+2 I_{4}-4 \tau I_{2}\right)-I_{3}^{2}+2\left(I_{1}-\tau\right) \tau^{2} \\
A\left(x_{1}, x_{2}, s\right)= & A_{0}(s)\left(x_{1} x_{2}-s\right)-I_{3}\left(x_{1}+x_{2}\right)+2 \tau\left(I_{1}-\tau\right)+2 \tau s \\
B\left(x_{1}, x_{2}, s\right)= & \left(x_{1}+x_{2}\right)^{2}-2 s-2 I_{1}+2 \tau .
\end{aligned}
$$


Denote by $m_{i}$ the zeros of polynomial $f$ and

$$
\begin{gathered}
P_{i}=\sqrt{\left(s_{1}-m_{i}\right)\left(s_{2}-m_{i}\right)} \quad i=1,2,3, \\
P_{i j}=P_{i} P_{j}\left(\frac{\dot{s}_{1}}{\left(s_{1}-m_{i}\right)\left(s_{1}-m_{j}\right)}+\frac{\dot{s}_{2}}{\left(s_{2}-m_{i}\right)\left(s_{2}-m_{j}\right)}\right)
\end{gathered}
$$

One can easily get

$$
P_{i}=\frac{\sqrt{A_{0}\left(m_{i}\right)}\left(x_{1} x_{2}-m_{i}\right)}{x_{1}-x_{2}}+\frac{-I_{3}\left(x_{1}+x_{2}\right)+2 \tau\left(I_{1}-\tau+m_{i}\right)}{\left(x_{1}-x_{2}\right) \sqrt{A_{0}\left(m_{i}\right)}}, \quad i=1,2,3 .
$$

Put

$$
\begin{aligned}
X & =\frac{x_{1} x_{2}}{x_{1}-x_{2}}, \quad Y=\frac{1}{x_{1}-x_{2}}, \\
Z & =\frac{-I_{3}\left(x_{1}+x_{2}\right)+2 \tau\left(I_{1}-\tau\right)}{x_{1}-x_{2}}, \\
n_{i} & =A_{0}\left(m_{i}\right)=2 m_{i}+2 I_{1}-2 \tau, \quad i=1,2,3 .
\end{aligned}
$$

The relations (3.50) can be rewritten as a system of linear equations

$$
\begin{aligned}
& X+Y m_{1}\left(\frac{2 \tau}{n_{1}}-1\right)+\frac{Z}{n_{1}}=\frac{P_{1}}{\sqrt{n_{1}}} \\
& X+Y m_{2}\left(\frac{2 \tau}{n_{2}}-1\right)+\frac{Z}{n_{2}}=\frac{P_{2}}{\sqrt{n_{2}}} \\
& X+Y m_{3}\left(\frac{2 \tau}{n_{3}}-1\right)+\frac{Z}{n_{3}}=\frac{P_{3}}{\sqrt{n_{3}}} .
\end{aligned}
$$

The solutions of the previous system are

$$
\begin{aligned}
& Y=-\sum_{i=1}^{3} \frac{\sqrt{n_{i}} P_{i}}{f^{\prime}\left(m_{i}\right)} \\
& X=-\sum_{i=1}^{3} \frac{P_{i} \sqrt{n_{i}}}{f^{\prime}\left(m_{i}\right)}\left(m_{j}+m_{k}+I_{1}-2 \tau\right) \\
& Z=\sum_{i=1}^{3} \frac{2 \sqrt{n_{i}} P_{i}}{f^{\prime}\left(m_{i}\right)}\left(\frac{n_{j} \cdot n_{k}}{4}+\tau\left(\tau-I_{1}\right)\right),
\end{aligned}
$$

with $(i, j, k)$-a cyclic permutation of $(1,2,3)$. 
Finally, we obtain

Proposition 3.3 The solutions of the system of differential Eq. (3.48) in terms of $P_{i}, P_{i j}$ functions are given with

$$
\begin{aligned}
M_{1} & =\frac{\sum_{i=1}^{3} \frac{2 \sqrt{n_{i}} P_{i}}{f^{\prime}\left(m_{i}\right)}\left(\frac{n_{j} \cdot n_{k}}{4}+\tau\left(\tau-I_{1}\right)\right)}{I_{3} \sum_{i=1}^{3} \frac{\sqrt{n_{i}} P_{i}}{f^{\prime}\left(m_{i}\right)}}+\frac{2 \tau\left(I_{1}-\tau\right)}{I_{3}} \\
M_{2} & =-\frac{1}{l \sum_{i=1}^{3} \frac{\sqrt{n_{i}} P_{i}}{f^{\prime}\left(m_{i}\right)}} \\
M_{3} & =\frac{2 i \sum_{k=1}^{3} \frac{n_{k} \sqrt{n_{i} n_{j}} P_{i j}}{f^{\prime}\left(m_{k}\right)}}{\sum_{i=1}^{3} \frac{\sqrt{n_{i}} P_{i}}{f^{\prime}\left(m_{i}\right)}}
\end{aligned}
$$

and

$$
\begin{aligned}
\gamma_{1}= & I_{2}+\frac{1}{8}\left(\frac{\sum_{k=1}^{3} \frac{n_{k} \sqrt{n_{i} n_{j}} P_{i j}}{f^{\prime}\left(m_{k}\right)}}{\sum_{i=1}^{3} \frac{\sqrt{n_{i}} P_{i}}{f^{\prime}\left(m_{i}\right)}}\right)^{2}-\frac{\sum_{i=1}^{3} \frac{P_{i} \sqrt{n_{i}}}{f^{\prime}\left(m_{i}\right)}\left(m_{j}+m_{k}+I_{1}-2 \tau\right)}{\sum_{i=1}^{3} \frac{P_{i} \sqrt{n_{i}}}{f^{\prime}\left(m_{i}\right)}} \\
\gamma_{2}= & -2 i \frac{\left(\sum_{k=1}^{3} \frac{n_{k} \sqrt{n_{i} n_{j}}}{f^{\prime}\left(m_{k}\right)} \frac{P_{i} P_{j}}{2}\right) \cdot\left(\sum_{i=1}^{3} \frac{\sqrt{n_{i}} P_{i}}{f^{\prime}\left(m_{i}\right)}\right)}{\left(\sum_{i=1}^{3} \frac{\sqrt{n_{i}} P_{i}}{f^{\prime}\left(m_{i}\right)}\right)^{2}} \\
& +2 i \frac{\left(\sum_{k=1}^{3} \frac{n_{k} \sqrt{n_{i} n_{j}} P_{i j}}{f^{\prime}\left(m_{k}\right)}\right) \cdot\left(\sum_{i=1}^{3} \frac{\sqrt{n_{i}}}{f^{\prime}\left(m_{i}\right)} \frac{P_{k} P_{i k}-P_{j} P_{i j}}{2\left(m_{j}-m_{k}\right)}\right)}{\left(\sum_{i=1}^{3} \frac{\sqrt{n_{i}} P_{i}}{f^{\prime}\left(m_{i}\right)}\right)^{2}} \\
\gamma_{3}= & \frac{\sum_{k=1}^{3} \frac{\sqrt{n_{i} n_{j}} P_{i j}}{f^{\prime}\left(m_{k}\right)}}{2 l}
\end{aligned}
$$

The formulae expressing $P_{i}, P_{i j}$ in terms of the theta-functions are given Kowalevski (1889). This gives the explicit formulae for the elasticae.

\section{The Lagrange Bitop and the $n$-Dimensional Hess-Appel'rot Systems}

\subsection{Higher-Dimensional Generalizations of Rigid Body Dynamics}

In 1966, in his seminal paper Arnold (1966), Arnold observed that two very important examples of the equations of motion, the ones of the Euler top and the Euler equations of the motion of inviscid incompressible fluid can be seen in a unified way and interpreted as the equations of the geodesic flows on a corresponding Lie group. The Riemannian metric is given by the kinetic energy. In the case of the Euler top, the Lie group is $S O(3)$ and the Riemannian metric, given by the Hamiltonian $2 H=\langle M, \Omega\rangle$ is left invariant. In the case of the fluid flow, the Lie group is a group 
of the volume-preserving diffeomorphisms and the metric is right-invariant. Starting from that observation, Arnold derived the equations of the geodesic flows of a left invariant metric on an arbitrary Lie group — and the Euler-Arnold equations emerged. The left invariance of the metric implies, for example, that the equations of the Euler top are written in the Lax form $\dot{M}=[M, \Omega]$, and hence one gets the family of the first integrals $\operatorname{tr}\left(M^{k}\right)$. The importance of Arnold's result is highlighted by the fact that many of the equations that appear in Physics can be represented as the Euler-Arnold equations.

The first ideas for constructing the higher-dimensional generalizations of the Euler top go back to the XIX century. Using some ideas of Cayley, Frahm presented the equations of the $n$-dimensional Euler top in 1874. He also constructed the family of the first integrals. However, the number of the first integrals was not enough to prove the integrability for $n>4$ (see Frahm 1874; Schottky 1891). In Manakov (1976) (not being aware of the results of Frahm) found an L-A pair for a wider class of metrics on $S O(n)$ given by $M_{i j}=\frac{a_{i}-a_{j}}{b_{i}-b_{j}} \Omega_{i j}$, and showed that this class belongs to the class considered by Dubrovin (1977). Hence, the solutions can be expressed in theta functions.

Arnold's observation was a starting point for a wide class of generalizations of the rigid body motion. For some of them see for example (Belokolos et al. 1994; Fedorov and Kozlov 1995; Trofimov and Fomenko 1995) and references therein.

Let us consider motion of $N$ points in $\mathbb{R}^{n}$ such that the distance between each two of them is constant in time. As an analogy with the three-dimensional case, we have two reference frames: the fixed and the moving ones. In the moving reference frame, the velocity of the point $A$ is:

$$
V_{A}(t)=B^{-1} \dot{q}_{A}(t)=B^{-1} \dot{B} Q_{A}=\Omega(t) Q_{A}
$$

where again $Q_{A}$ represents the radius vector of the point $A$, and $\Omega$ is skew-symmetric matrix $(\Omega \in \operatorname{so}(n))$ representing the angular velocity of the body in the moving reference frame. The angular momentum is a skew-symmetric matrix defined by

$$
\begin{aligned}
M & =\int_{B}\left(V Q^{t}-Q V^{t}\right) d m=\int_{B}\left(\Omega Q Q^{t}-Q Q^{t} \Omega^{t}\right) d m \\
& =\int_{B}\left(\Omega Q Q^{t}+Q Q^{t} \Omega\right) d m=\Omega I+I \Omega,
\end{aligned}
$$

where $I=\int_{B} Q Q^{t} d m$ is a constant symmetric matrix called the mass tensor of the body (see Fedorov and Kozlov 1995) and integration goes over the body $B$.

If one chooses the basis in which $I=\operatorname{diag}\left(I_{1}, \ldots, I_{n}\right)$, the coordinates of angular momentum are $M_{i j}=\left(I_{i}+I_{j}\right) \Omega_{i j}$.

The kinetic energy is

$$
T=\frac{1}{2} \int_{B}\langle\dot{Q}, \dot{Q}\rangle d m=\frac{1}{2} \int_{B}\langle\Omega Q, \Omega Q\rangle d m
$$


Since it is a homogeneous quadratic form of angular velocity $\Omega$, one has $\left\langle\frac{\partial T}{\partial \Omega}, \Omega\right\rangle=2 T$ where $\langle A, B\rangle=-\frac{1}{2} \operatorname{Trace}(A B)$ is an invariant scalar product on $s o(n)$. One gets

$$
\frac{\partial T}{\Omega_{k l}}=\sum_{m}\left(\Omega_{k m} I_{m l}+I_{k m} \Omega_{m l}\right),
$$

or $\frac{\partial T}{\partial Q}=M$ and finally

$$
T=\frac{1}{2}\langle M, \Omega\rangle
$$

The Lie group $E(3)$ can be regarded as a semidirect product of the Lie groups $S O(3)$ and $\mathbb{R}^{3}$. The product in the group given by

$$
\left(A_{1}, r_{1}\right) \cdot\left(A_{2}, r_{2}\right)=\left(A_{1} A_{2}, r_{1}+A_{1} r_{2}\right)
$$

corresponds to the composition of two isometric transformations of the Euclidian space. The Lie algebra $e(3)$ is a semidirect product of $\mathbb{R}^{3}$ and $s o(3)$. Using isomorphism between the Lie algebras $s o(3)$ and $\mathbb{R}^{3}$, given by (2.1), one concludes that $e(3)$ is also isomorphic to the semidirect product $s=s o(3) \times{ }_{a d} s o(3)$. The commutator in $s$ is given by:

$$
\left[\left(a_{1}, b_{1}\right),\left(a_{2}, b_{2}\right)\right]=\left(\left[a_{1}, a_{2}\right],\left[a_{1}, b_{2}\right]+\left[b_{1}, a_{2}\right]\right) .
$$

One concludes, that there are two natural higher-dimensional generalizations of Eq. (2.2). The first one is on the Lie algebra $e(n)$ that is a semidirect product of $s o(n)$ and $\mathbb{R}^{n}$. The other one is on semidirect product $s=\operatorname{so}(n) \times{ }_{a d} \operatorname{so}(n)$.

\subsection{The Heavy Rigid Body Equations on $e(n)$}

The Euler-Arnold equations of motion of a heavy rigid body fixed at a point on $e(n)$ are (see Belyaev 1981; Trofimov and Fomenko 1995; Jovanović 2007 and references therein):

$$
\dot{M}=[M, \Omega]+\Gamma \wedge X, \quad \dot{\Gamma}=-\Omega \Gamma .
$$

Here $M$ and $\Omega$ are connected by $M=I \Omega+\Omega I$. The $n$-dimensional vectors $\Gamma$, fixed in the space, and $X$, fixed in the body, are generalizations of the unit vertical vector and of the radius vector of the center of masses respectively.

The $n$-dimensional Lagrange top on $e(n)$ is defined by Belyaev in Belyaev (1981) by conditions:

$$
I=\operatorname{diag}\left(I_{1}, I_{1}, \ldots, I_{1}, I_{n}\right), \quad X=\left(0,0, \ldots, 0, x_{n}\right)
$$

Belyaev also proved the integrability of these systems. 


\subsection{The Heavy Rigid Body Equations on $s=\operatorname{so}(n) \times{ }_{a d} s o(n)$}

The equations of the motion of a rigid body on semidirect product $s=\operatorname{so}(n) \times$ ad $\operatorname{so}(n)$ were given by Ratiu (1982):

$$
\dot{M}=[M, \Omega]+[\Gamma, \chi], \quad \dot{\Gamma}=[\Gamma, \Omega]
$$

Here $M \in \operatorname{so}(n)$ is the angular momentum, $\Omega \in \operatorname{so}(n)$ is the angular velocity, $\chi \in$ $s o(n)$ is a given constant matrix (describing a generalized center of the mass), $\Gamma \in$ so(n). Angular momentum $M$ and $\Omega$ are connected by $M=I \Omega+\Omega I$. If the matrix $I$ is diagonal, $I=\operatorname{diag}\left(I_{1}, \ldots, I_{n}\right)$, then $M_{i j}=\left(I_{i}+I_{j}\right) \Omega_{i j}$. The Lie algebra $s$ is the Lie algebra of Lie group $S=S O(n) \times{ }_{A d} S O(n)$ that is semidirect product of $S O(n)$ and $s o(n)$ (here $s o(n)$ is considered as the Abelian Lie group). The group product in $S$ is $\left(A_{1}, b_{1}\right) \cdot\left(A_{2}, b_{2}\right)=\left(A_{1} A_{2}, b_{1}+A d_{A_{1}} b_{2}\right)$.

Ratiu proved that Eq. (4.3) are Hamiltonian in the Lie-Poisson structure on coadjoint orbits of group $S$ given by:

$$
\begin{aligned}
\{\tilde{f}, \tilde{g}\}(\mu, v)= & -\mu\left(\left[d_{1} f(\mu, v), d_{1} g(\mu, v)\right]\right) \\
& -v\left(\left[d_{1} f(\mu, v), d_{2} g(\mu, v)\right]\right) \\
& -v\left(\left[d_{2} f(\mu, v), d_{1} g(\mu, v)\right]\right),
\end{aligned}
$$

where $\tilde{f}, \tilde{g}$ are restrictions of functions $f$ and $g$ on orbits of coadjoint action and $d_{i} f$ are partial derivatives of $d f$. On so $(n)$ a bilinear symmetric nondegenerate biinvariant (i.e. $k([\xi, \eta], \zeta)=k(\xi,[\eta, \zeta]))$ two form exist, which can be extended to $s$ as well:

$$
k_{s}\left(\left(\xi_{1}, \eta_{1}\right),\left(\xi_{2}, \eta_{2}\right)\right)=k\left(\xi_{1}, \eta_{2}\right)+k\left(\xi_{2}, \eta_{1}\right) .
$$

Hence, one can identify $s^{*}$ and $s$. Then, the Poisson structure (4.4) can be written in the form

$$
\begin{aligned}
\{\tilde{f}, \tilde{g}\}(\xi, \eta)= & -k\left(\xi,\left[\left(\operatorname{grad}_{2} f\right)(\xi, \eta),\left(\operatorname{grad}_{1} g\right)(\xi, \eta)\right]\right) \\
& -k\left(\xi,\left[\left(\operatorname{grad}_{1} f\right)(\xi, \eta),\left(\operatorname{grad}_{2} g\right)(\xi, \eta)\right]\right) \\
& -k\left(\eta,\left[\left(\operatorname{grad}_{2} f\right)(\xi, \eta),\left(\operatorname{grad}_{2} g\right)(\xi, \eta)\right]\right),
\end{aligned}
$$

where $\operatorname{grad}_{i}$ are $k$-gradients in respect to the $i$-th coordinate.

In Ratiu (1982), the Lagrange case was defined by $I_{1}=I_{2}=a, I_{3}=\cdots=I_{n}=$ $b, \chi_{12}=-\chi_{21} \neq 0, \chi_{i j}=0,(i, j) \notin\{(1,2),(2,1)\}$. The completely symmetric case was defined there by $I_{1}=\cdots=I_{n}=a$, where $\chi \in s o(n)$ is an arbitrary constant matrix. It was shown in Ratiu (1982) that Eq. (4.3) in these cases could be represented by the following L-A pair:

$$
\frac{d}{d t}\left(\lambda^{2} C+\lambda M+\Gamma\right)=\left[\lambda^{2} C+\lambda M+\Gamma, \lambda \chi+\Omega\right],
$$

where in the Lagrange case $C=(a+b) \chi$, and in the symmetric case $C=2 a \chi$. 


\subsection{Four-Dimensional Rigid Body Motion}

To any $3 \times 3$ skew-symmetric matrix one assigns one vector in three-dimensional space using isomorphism between $\mathbb{R}^{3}$ and $s o(3)$. Using the the isomorphism between so(4) and $s o(3) \times s o(3)$, one can assign two three-dimensional vectors $A_{1}$ and $A_{2}$ to $(4 \times 4)$-skew-symmetric matrix $A$.

Vectors $A_{1}$ and $A_{2}$ are defined by:

$$
A_{1}=\frac{A_{+}+A_{-}}{2}, \quad A_{2}=\frac{A_{+}-A_{-}}{2},
$$

where $A_{+}, A_{-} \in \mathbb{R}^{3}$ correspond to $A_{i j} \in \operatorname{so}(4)$ according to:

$$
\left(A_{+}, A_{-}\right) \rightarrow\left(\begin{array}{cccc}
0 & -A_{+}^{3} & A_{+}^{2} & -A_{-}^{1} \\
A_{+}^{3} & 0 & -A_{+}^{1} & -A_{-}^{2} \\
-A_{+}^{2} & A_{+}^{1} & 0 & -A_{-}^{3} \\
A_{-}^{1} & A_{-}^{2} & A_{-}^{3} & 0
\end{array}\right)
$$

Here $A_{ \pm}^{j}$ are the $j$-th coordinates of the vector $A_{ \pm}$.

By direct calculations, we check that vectors $2 A_{1} \times B_{1}$ and $2 A_{2} \times B_{2}$ correspond to commutator $[A, B]$, if vectors $A_{1}, A_{2}$ and $B_{1}, B_{2}$ correspond to $A$ and $B$ respectively.

Consequently, equations of motion (4.3) on $\operatorname{so}(4) \times \operatorname{so}(4)$ can be written as:

$$
\begin{array}{ll}
\dot{M}_{1}=2\left(M_{1} \times \Omega_{1}+\Gamma_{1} \times \chi_{1}\right) & \dot{\Gamma}_{1}=2\left(\Gamma_{1} \times \Omega_{1}\right) \\
\dot{M}_{2}=2\left(M_{2} \times \Omega_{2}+\Gamma_{2} \times \chi_{2}\right) & \dot{\Gamma}_{2}=2\left(\Gamma_{2} \times \Omega_{2}\right)
\end{array}
$$

Recall that $M=I \Omega+\Omega I$. The matrix elements of the mass tensor of the body $I$ are $I_{k l}=\int_{B} Q_{k} Q_{l} d m, k, l=1, \ldots, 4$. Choose the coordinates $\left(X_{1}, X_{2}, X_{3}, X_{4}\right)$ of the moving reference frame in which $I$ has diagonal form $I=\operatorname{diag}\left(I_{1}, I_{2}, I_{3}, I_{4}\right)$. Then, for example $I_{1}=\int_{B} X_{1}^{2} d m, I_{2}=\int_{B} X_{2}^{2} d m, \int_{B} X_{1} X_{2} d m=0$ etc. In the threedimensional case the moments of inertia were defined with respect to the line through the fixed point $O$. We derive the angular velocity $\Omega$ as a skew-symmetric matrix the elements of which correspond to the rotations in two-dimensional coordinate planes. Hence, here it is natural to define the moments of inertia of the body with respect to the two-dimensional planes through the fixed point. For example the moment of inertia with respect to the plane $X_{1} O X_{2}$ is $I_{1}+I_{2}$, and $M_{12}=\left(I_{1}+I_{2}\right) \Omega_{12}$, etc.

Here we observe a complete analogy with the three-dimensional case. For example, the moment of inertia with respect to $O Z$ axis $I_{33}=\int_{B}\left(X^{2}+Y^{2}\right) d m$ consists of two addend $\int_{B} X^{2} d m$ and $\int_{B} Y^{2} d m$ that are diagonal elements of the mass tensor of the body.

For vectors $M_{+}$and $M_{-}$one has

$$
\begin{aligned}
& M_{+}=\left(\left(I_{2}+I_{3}\right) \Omega_{+}^{1},\left(I_{3}+I_{2}\right) \Omega_{+}^{2},\left(I_{3}+I_{1}\right) \Omega_{+}^{3}\right)=I_{+} \Omega_{+} \\
& M_{-}=\left(\left(I_{1}+I_{4}\right) \Omega_{-}^{1},\left(I_{2}+I_{4}\right) \Omega_{-}^{2},\left(I_{3}+I_{4}\right) \Omega_{-}^{3}\right)=I_{-} \Omega_{-} .
\end{aligned}
$$


Finally, one can calculate

$$
\begin{aligned}
& M_{1}=\frac{1}{2}\left(\left(I_{+}+I_{-}\right) \Omega_{1}+\left(I_{+}-I_{-}\right) \Omega_{2}\right) \\
& M_{2}=\frac{1}{2}\left(\left(I_{+}-I_{-}\right) \Omega_{1}+\left(I_{+}+I_{-}\right) \Omega_{2}\right)
\end{aligned}
$$

At a glance it looks that (4.7) are equations of motion of two independent three-dimensional rigid bodies. However, the formulas (4.8) show that they are not independent and that each of $M_{1}, M_{2}$ depends on both $\Omega_{1}$ and $\Omega_{2}$.

\subsection{The Lagrange Bitop: Definition and a Lax Representation}

Generalizing the Lax representation of the Hess-Appel'rot system, a new completely integrable four-dimensional rigid body system is established in Dragović and Gajić (2001). A detailed classical and algebro-geometric integration were presented in Dragović and Gajić (2004).

The Lagrange bitop is a four-dimensional rigid body system on the semidirect product $s o(4) \times_{a d} s o(4)$ defined by (see Dragović and Gajić 2001, 2004):

$$
\begin{aligned}
& I_{1}=I_{2}=a \\
& I_{3}=I_{4}=b
\end{aligned} \quad \text { and } \quad \chi=\left(\begin{array}{cccc}
0 & \chi_{12} & 0 & 0 \\
-\chi_{12} & 0 & 0 & 0 \\
0 & 0 & 0 & \chi_{34} \\
0 & 0 & -\chi_{34} & 0
\end{array}\right)
$$

with the conditions $a \neq b, \chi_{12}, \chi_{34} \neq 0,\left|\chi_{12}\right| \neq\left|\chi_{34}\right|$.

We have the following proposition:

Proposition 4.1 (Dragović and Gajić 2001, 2004) The equations of motion (4.3) under conditions (4.9) have an $L-A$ pair representation $\dot{L}(\lambda)=[L(\lambda), A(\lambda)]$, where

$$
L(\lambda)=\lambda^{2} C+\lambda M+\Gamma, \quad A(\lambda)=\lambda \chi+\Omega,
$$

and $C=(a+b) \chi$.

Let us briefly analyze the spectral properties of the matrices $L(\lambda)$. The spectral polynomial $p(\lambda, \mu)=\operatorname{det}(L(\lambda)-\mu \cdot 1)$ has the form

$$
p(\lambda, \mu)=\mu^{4}+P(\lambda) \mu^{2}+[Q(\lambda)]^{2},
$$

where

$$
\begin{aligned}
& P(\lambda)=A \lambda^{4}+B \lambda^{3}+D \lambda^{2}+E \lambda+F, \\
& Q(\lambda)=G \lambda^{4}+H \lambda^{3}+I \lambda^{2}+J \lambda+K .
\end{aligned}
$$


Their coefficients

$$
\begin{aligned}
A & =C_{12}^{2}+C_{34}^{2}=\left\langle C_{+}, C_{+}\right\rangle+\left\langle C_{-}, C_{-}\right\rangle, \\
B & =2 C_{34} M_{34}+2 C_{12} M_{12}=2\left(\left\langle C_{+}, M_{+}\right\rangle+\left\langle C_{-}, M_{-}\right\rangle\right), \\
D & =M_{13}^{2}+M_{14}^{2}+M_{23}^{2}+M_{12}^{2}+M_{34}^{2}+2 C_{12} \Gamma_{12}+2 C_{34} \Gamma_{34} \\
& =\left\langle M_{+}, M_{+}\right\rangle+\left\langle M_{-}, M_{-}\right\rangle+2\left(\left\langle C_{+}, \Gamma_{+}\right\rangle+\left\langle C_{-}, \Gamma_{-}\right\rangle\right), \\
E & =2 \Gamma_{12} M_{12}+2 \Gamma_{13} M_{13}+2 \Gamma_{14} M_{14}+2 \Gamma_{23} M_{23}+2 \Gamma_{24} M_{24}+2 \Gamma_{34} M_{34} \\
& =2\left(\left\langle\Gamma_{+}, M_{+}\right\rangle+\left\langle\Gamma_{-}, M_{-}\right\rangle\right), \\
F & =\Gamma_{12}^{2}+\Gamma_{13}^{2}+\Gamma_{14}^{2}+\Gamma_{23}^{2}+\Gamma_{24}^{2}+\Gamma_{34}^{2}=\left\langle\Gamma_{+}, \Gamma_{+}\right\rangle+\left\langle\Gamma_{-}, \Gamma_{-}\right\rangle, \\
G & =C_{12} C_{34}=\left\langle C_{+}, C_{-}\right\rangle, \\
H & =C_{34} M_{12}+C_{12} M_{34}=\left\langle C_{+}, M_{-}\right\rangle+\left\langle C_{-}, M_{+}\right\rangle, \\
I & =C_{34} \Gamma_{12}+\Gamma_{34} C_{12}+M_{12} M_{34}+M_{23} M_{14}-M_{13} M_{24} \\
& =\left\langle C_{+}, \Gamma_{-}\right\rangle+\left\langle C_{-}, \Gamma_{+}\right\rangle+\left\langle M_{+}, M_{-}\right\rangle, \\
J & =M_{34} \Gamma_{12}+M_{12} \Gamma_{34}+M_{14} \Gamma_{23}+M_{23} \Gamma_{14}-\Gamma_{13} M_{24}-\Gamma_{24} M_{13} \\
& =\left\langle M_{+}, \Gamma_{-}\right\rangle+\left\langle M_{-}, \Gamma_{+}\right\rangle, \\
K & =\Gamma_{34} \Gamma_{12}+\Gamma_{23} \Gamma_{14}-\Gamma_{13} \Gamma_{24}=\left\langle\Gamma_{+}, \Gamma_{-}\right\rangle .
\end{aligned}
$$

are integrals of motion of the system (4.3), (4.9). Here $M_{+}, M_{-} \in \mathbb{R}^{3}$ are defined by (4.6) (similar for other vectors). System (4.3), (4.9) is Hamiltonian with the Hamiltonian function

$\mathcal{H}=\frac{1}{2}\left(M_{13} \Omega_{13}+M_{14} \Omega_{14}+M_{23} \Omega_{23}+M_{12} \Omega_{12}+M_{34} \Omega_{34}\right)+\chi_{12} \Gamma_{12}+\chi_{34} \Gamma_{34}$.

The algebra $s o(4) \times s o(4)$ is 12 -dimensional. The general orbits of the coadjoint action are 8-dimensional. According to Ratiu (1982), the Casimir functions are coefficients of $\lambda^{0}, \lambda, \lambda^{4}$ in the polynomials $[\operatorname{det} L(\lambda)]^{1 / 2}$ and $-\frac{1}{2} \operatorname{Tr}(L(\lambda))^{2}$. One calculates:

$[\operatorname{det} L(\lambda)]^{1 / 2}=G \lambda^{4}+H \lambda^{3}+I \lambda^{2}+J \lambda+K, \quad-\frac{1}{2} \operatorname{Tr}(L(\lambda))^{2}=A \lambda^{4}+E \lambda+F$.

Thus, Casimir functions are $J, K, E, F$. Nontrivial integrals of motion are $B, D, H, I$. As one can check easily, they are in involution. When $\left|\chi_{12}\right|=\left|\chi_{34}\right|$, then $2 H=B$ or $2 H=-B$ and there are only 3 independent integrals in involution. Thus,

Proposition 4.2 (Dragović and Gajić 2004) For $\left|\chi_{12}\right| \neq\left|\chi_{34}\right|$, system (4.3), (4.9) is completely integrable in the Liouville sense.

System (4.3), (4.9) doesn't fall in any of the families defined by Ratiu (1982) and together with them it makes complete list of systems with the $L$ operator of the form

$$
L(\lambda)=\lambda^{2} C+\lambda M+\Gamma .
$$


More precisely, if $\chi_{12} \neq 0$, then the Euler-Poisson Eq. (4.3) could be written in the form (4.10) (with arbitrary $C$ ) if and only if Eq. (4.3) describe the generalized symmetric case, the generalized Lagrange case or the Lagrange bitop, including the case $\chi_{12}= \pm \chi_{34}$ (Dragović and Gajić 2001).

\subsubsection{Classical Integration}

For classical integration we will use Eq. (4.7). On can calculate that

$$
\chi_{1}=\left(0,0,-\frac{1}{2}\left(\chi_{12}+\chi_{34}\right)\right), \quad \chi_{2}=\left(0,0,-\frac{1}{2}\left(\chi_{12}-\chi_{34}\right)\right)
$$

and also

$$
\begin{aligned}
& M_{1}=\left((a+b) \Omega_{(1) 1},(a+b) \Omega_{(1) 2},(a+b) \Omega_{(1) 3}+(a-b) \Omega_{(2) 3}\right) \\
& M_{2}=\left((a+b) \Omega_{(2) 1},(a+b) \Omega_{(2) 2},(a-b) \Omega_{(1) 3}+(a+b) \Omega_{(2) 3}\right) .
\end{aligned}
$$

If we denote $\Omega_{1}=\left(p_{1}, q_{1}, r_{1}\right), \Omega_{2}=\left(p_{2}, q_{2}, r_{2}\right)$, then the first group of the Eq. (4.7) becomes

$$
\begin{aligned}
\dot{p}_{1}-m q_{1} r_{2} & =-n_{1} \Gamma_{(1) 2}, \dot{p}_{2}-m q_{2} r_{1}=-n_{2} \Gamma_{(2) 2} \\
\dot{q}_{1}+m p_{1} r_{2} & =n_{1} \Gamma_{(1) 1}, \dot{q}_{2}+m p_{2} r_{1}=n_{2} \Gamma_{(2) 1} \\
(a+b) \dot{r}_{1}+(a-b) \dot{r}_{2} & =0,(a-b) \dot{r}_{1}+(a+b) \dot{r}_{2}=0
\end{aligned}
$$

where

$$
m=-\frac{2(a-b)}{a+b}, \quad n_{1}=-\frac{2 \chi(1) 3}{a+b}, \quad n_{2}=-\frac{2 \chi(2) 3}{a+b} .
$$

The integrals of motion are for $i=1,2$ :

$$
\begin{aligned}
& (a+b) \alpha_{i} \chi_{(i) 3}=f_{i 1} \\
& (a+b)\left[(a+b)\left(p_{i}^{2}+q_{i}^{2}\right)+(a+b) \alpha_{i}^{2}+2 \chi_{(i) 3} \Gamma_{(i) 3}\right]=f_{i 2} \\
& (a+b) p_{i} \Gamma_{(i) 1}+(a+b) q_{i} \Gamma_{(i) 2}+(a+b) \alpha_{i} \Gamma_{(i) 3}=f_{i 3} \\
& \Gamma_{(i) 1}^{2}+\Gamma_{(i) 2}^{2}+\Gamma_{(i) 3}^{2}=1
\end{aligned}
$$

where

$$
\begin{aligned}
\alpha_{1} & =\frac{(a+b) r_{1}+(a-b) r_{2}}{a+b} \quad \alpha_{2}=\frac{(a+b) r_{2}+(a-b) r_{1}}{a+b} \\
a_{i} & =\frac{\alpha_{i}^{2}(a+b)^{2}-f_{i 2}}{(a+b)^{2}} \quad i=1,2 .
\end{aligned}
$$


Introducing $\rho_{i}, \sigma_{i}$, defined with $p_{i}=\rho_{i} \cos \sigma_{i}, q_{i}=\rho_{i} \sin \sigma_{i}$, after calculations, one gets

$$
\begin{aligned}
& \rho_{1}^{2} \dot{\sigma}_{1}+m r_{2} \rho_{1}^{2}=n_{1}\left(\frac{f_{13}}{a+b}-\alpha_{1} \Gamma_{(1) 3}\right) \\
& {\left[\left(\rho_{i}^{2}\right)^{\cdot}\right]^{2}=4 n_{i}^{2} \rho_{i}^{2}\left[1-\frac{1}{n_{i}^{2}}\left(a_{i}+\rho_{i}^{2}\right)^{2}\right]-4 n_{i}^{2}\left(\frac{f_{i 3}}{a+b}-\alpha_{i} a_{i}-\frac{\alpha_{i}}{n_{i}} \rho_{i}^{2}\right)^{2}, \quad i=1,2} \\
& \rho_{2}^{2} \dot{\sigma}_{2}+m r_{1} \rho_{2}^{2}=n_{2}\left(\frac{f_{23}}{a+b}-\alpha_{2} \Gamma_{(2) 3}\right) .
\end{aligned}
$$

Let us denote $u_{1}=\rho_{1}^{2}, u_{2}=\rho_{2}^{2}$. From (4.12) we have

$$
\begin{gathered}
\dot{u}_{i}^{2}=P_{i}\left(u_{i}\right), \quad i=1,2, \\
P_{i}(u)=-4 u^{3}-4 u^{2} B_{i}+4 u C_{i}+D_{i}, \quad i=1,2 \\
B_{i}=2 a_{i}+\alpha_{i}^{2}, \quad C_{i}=n_{i}^{2}-a_{i}^{2}-4 \frac{\alpha_{i} \chi_{(i) 3} f_{i 3}}{(a+b)^{2}}-2 \alpha_{i}^{2} a_{i}, \\
D_{i}=-4\left(\frac{2 \chi_{(i) 3} f_{i 3}}{(a+b)^{2}}+\alpha_{i} a_{i}\right)^{2}, \quad i=1,2 .
\end{gathered}
$$

From the previous relations, we have

$$
\int \frac{d u_{1}}{\sqrt{P_{1}\left(u_{1}\right)}}=t, \quad \int \frac{d u_{2}}{\sqrt{P_{2}\left(u_{2}\right)}}=t .
$$

So, the integration of the Lagrange bitop leads to the functions associated with the elliptic curves $E_{1}, E_{2}$ where $E_{i}=E_{i}\left(\alpha_{i}, a_{i}, \chi_{(i) 3}, f_{i 2}, f_{i 3}\right)$ are given with:

$$
E_{i}: y^{2}=P_{i}(u)
$$

Equation (4.7) are very similar to those for the classical Lagrange system. However, the system doesn't split on two independent Lagrangian systems.

\subsubsection{Properties of the Spectral Curve}

The spectral curve of the Lagrange bitop is given by:

$$
\mathcal{C}: \mu^{4}+P(\lambda) \mu^{2}+[Q(\lambda)]^{2}=0
$$

where $P$ and $Q$ are given by (4.11).

There is an involution $\sigma:(\lambda, \mu) \rightarrow(\lambda,-\mu)$ on the spectral curve which corresponds to the skew symmetry of the matrix $L(\lambda)$. Denote the factor-curve by $\mathcal{C}_{1}=\mathcal{C} / \sigma$. 
Lemma 4.1 (Dragović and Gajić 2004) The basic properties of the spectral curve are:

- The curve $\mathcal{C}_{1}$ is a smooth hyperelliptic curve of genus three: $g\left(\mathcal{C}_{1}\right)=3$. The spectral curve $\mathcal{C}$ is a double covering of $\mathcal{C}_{1}$. The arithmetic genus of $\mathcal{C}$ is $g_{a}(\mathcal{C})=9$.

- The spectral curve $\mathcal{C}$ has four ordinary double points $S_{i}, i=1, \ldots, 4$. The genus of its normalization $\tilde{\mathcal{C}}$ is five.

- The singular points $S_{i}$ of the curve $\mathcal{C}$ are fixed points of the involution $\sigma$. The involution $\sigma$ exchanges the two branches of $\mathcal{C}$ at $S_{i}$.

- The involution $\sigma$ extended to the normalization $\tilde{\mathcal{C}}$ is fixed-points free.

The general theories describing the isospectral deformations for polynomials with matrix coefficients were developed by Dubrovin (1977), Dubrovin et al. (1976, 2001) in the middle of 70's and by Adler and van Moerbeke (1980) a few years later. Dubrovin's approach was based on the Baker-Akhiezer function and it was applied in rigid body problems in Manakov (1976), Bogoyavlensky (1984). Application of the Adler van Moerbeke approach to rigid body problems were given in Adler and van Moerbeke (1980), Ratiu (1982), Ratiu and van Moerbeke (1982), Adler et al. (2004).

However, non of these two theories can be directly applied for an algebro-geometric integration of the Lagrange bitop.

The detailed algebro-geometric integration procedure of the system is given in Dragović and Gajić (2004). Analysis of the spectral curve and the Baker-Akhiezer function shows that the dynamics of the system is related to a certain Prym variety $\Pi$ that corresponds to the double covering defined by the involution $\sigma$ and to evolution of divisors of some meromorphic differentials $\Omega_{j}^{i}$. It appears that

$$
\Omega_{2}^{1}, \Omega_{1}^{2}, \Omega_{4}^{3}, \Omega_{3}^{4}
$$

are holomorphic during the whole evolution. Compatibility of this requirement with the dynamics puts a strong constraint on the spectral curve: its theta divisor should contain some torus. In the case presented here such a constraint appears to be satisfied according to Mumford's relation from Mumford (1974) (see Dragović and Gajić 2004, formula (2)). These conditions create a new situation from the point of view of then existing integration techniques. We call such systems the isoholomorphic systems.

We characterize the class of isoholomorphic integrable systems by the following properties:

(a) There exists an involution on the (normalized) spectral curve without fixed points.

(b) The standard Krichever axioms for the Baker-Akhiezer function are not satisfied.

(c) The Mumford relation on the theta-divisors as a geometric realization of the dynamics is satisfied.

For more detail see Dragović and Gajić (2004). Some other examples of the isoholomorphic systems were presented in Dragović et al. (2009).

Several years after Dragović and Gajić (2004) the isoholomorphic systems were essentially rediscovered by Grushevsky and Krichever (2010) and these systems played the decisive role in their remarkable solution of an important and delicate algebrogeometric problem of characterization of the Prym varieties. 


\subsection{Four-Dimensional Hess-Appel'rot Systems}

The starting point for construction of generalization of the Hess-Appel'rot system was Zhukovski's geometric interpretation given in Sect. 2.5. Having it in mind, in Dragović and Gajić (2006) the higher-dimensional Hess-Appel'rot systems are defined. First we will consider the four-dimensional case on $s o(4) \times s o(4)$. We will consider metric given with $\Omega=J M+M J$.

Definition 4.1 (Dragović and Gajić 2006) The four-dimensional Hess-Appel'rot system is described by Eq. (4.3) and satisfies the conditions:

1.

$$
\Omega=M J+J M, \quad J=\left(\begin{array}{cccc}
J_{1} & 0 & J_{13} & 0 \\
0 & J_{1} & 0 & J_{24} \\
J_{13} & 0 & J_{3} & 0 \\
0 & J_{24} & 0 & J_{3}
\end{array}\right)
$$

2.

$$
\chi=\left(\begin{array}{cccc}
0 & \chi_{12} & 0 & 0 \\
-\chi_{12} & 0 & 0 & 0 \\
0 & 0 & 0 & \chi_{34} \\
0 & 0 & -\chi_{34} & 0
\end{array}\right)
$$

The invariant surfaces are determined in the following lemma.

Lemma 4.2 (Dragović and Gajić 2006) For the four-dimensional Hess-Appel'rot system, the following relations take place:

$$
\begin{aligned}
& \dot{M}_{12}=J_{13}\left(M_{13} M_{12}+M_{24} M_{34}\right)+J_{24}\left(M_{13} M_{34}+M_{12} M_{24}\right), \\
& \dot{M}_{34}=J_{13}\left(-M_{13} M_{34}-M_{12} M_{24}\right)+J_{24}\left(-M_{13} M_{12}-M_{24} M_{34}\right) .
\end{aligned}
$$

In particular, if $M_{12}=M_{34}=0$ hold at the initial moment, then the same relations are satisfied during the evolution in time.

Thus, in the four-dimensional Hess-Appel'rot case, there are two invariant relations

$$
M_{12}=0, \quad M_{34}=0 .
$$

Let us now present another definition of the four-dimensional Hess-Appel'rot conditions, starting from a basis where the matrix $J$ is diagonal in.

Let $\tilde{J}=\operatorname{diag}\left(\tilde{J}_{1}, \tilde{J}_{2}, \tilde{J}_{3}, \tilde{J}_{4}\right)$. 
Definition 4.2 (Dragović and Gajić 2006) The four-dimensional Hess-Appel'rot system is described by the Eq. (4.3) and satisfies the conditions:

1.

$$
\Omega=M \tilde{J}+\tilde{J} M, \quad \tilde{J}=\operatorname{diag}\left(\tilde{J}_{1}, \tilde{J}_{2}, \tilde{J}_{3}, \tilde{J}_{4}\right)
$$

2.

$$
\tilde{\chi}=\left(\begin{array}{cccc}
0 & \tilde{\chi}_{12} & 0 & \tilde{\chi}_{14} \\
-\tilde{\chi}_{12} & 0 & \tilde{\chi}_{23} & 0 \\
0 & -\tilde{\chi}_{23} & 0 & \tilde{\chi}_{34} \\
-\tilde{\chi}_{14} & 0 & -\tilde{\chi}_{34} & 0
\end{array}\right)
$$

3.

$$
\begin{aligned}
& \tilde{J}_{3}-\tilde{J}_{4}=\tilde{J}_{2}-\tilde{J}_{1}, \\
& \frac{\tilde{J}_{3}-\tilde{J}_{1}}{\sqrt{1+t_{1}^{2}}}=\frac{\tilde{J}_{4}-\tilde{J}_{2}}{\sqrt{1+t_{2}^{2}}}
\end{aligned}
$$

where

$$
\begin{aligned}
t_{1} & :=\frac{2\left(\tilde{\chi}_{14} \tilde{\chi}_{34}-\tilde{\chi}_{12} \tilde{\chi}_{23}\right)}{\tilde{\chi}_{14}^{2}-\tilde{\chi}_{34}^{2}+\tilde{\chi}_{12}^{2}-\tilde{\chi}_{23}^{2}}, \\
t_{2}:= & \frac{2\left(\tilde{\chi}_{14} \tilde{\chi}_{12}-\tilde{\chi}_{23} \tilde{\chi}_{34}\right)}{-\tilde{\chi}_{14}^{2}-\tilde{\chi}_{34}^{2}+\tilde{\chi}_{12}^{2}+\tilde{\chi}_{23}^{2}} .
\end{aligned}
$$

Proposition 4.3 (Dragović and Gajić 2006) There exists a bi-correspondence between sets of data from Definitions 4.1 and 4.2.

Remark 4.1 1. In the case $J_{24} \neq 0, \chi_{34}=0$, there is an additional relation $\tilde{\chi}_{12} \tilde{\chi}_{34}+$ $\tilde{\chi}_{14} \tilde{\chi}_{23}=0$. It follows from the system

$$
\begin{aligned}
& \tilde{\chi}_{12} \sin \varphi+\tilde{\chi}_{23} \cos \varphi=0 \\
& \tilde{\chi}_{14} \sin \varphi-\tilde{\chi}_{34} \cos \varphi=0
\end{aligned}
$$

2. In the case $J_{24}=0, \chi_{34}=0$, additional relations are $\tilde{\chi}_{34}=\tilde{\chi}_{14}=0$, and the second relation from Definition 4.2 can be replaced by the relation

$$
\tilde{\chi}_{12} \sqrt{\tilde{J}_{2}-\tilde{J}_{1}}+\tilde{\chi}_{23} \sqrt{\tilde{J}_{3}-\tilde{J}_{2}}=0 .
$$

Theorem 4.1 (Dragović and Gajić 2006) The four-dimensional Hess-Appel'rot system has the following Lax representation 


$$
\begin{aligned}
& \dot{L}(\lambda)=[L(\lambda), A(\lambda)], \\
& L(\lambda)=\lambda^{2} C+\lambda M+\Gamma, \quad A(\lambda)=\lambda \chi+\Omega, \quad C=\frac{1}{J_{1}+J_{3}} \chi .
\end{aligned}
$$

One can calculate the spectral polynomial for the four-dimensional Hess-Appel'rot system:

$$
p(\lambda, \mu)=\operatorname{det}(L(\lambda)-\mu \cdot 1)=\mu^{4}+P(\lambda) \mu^{2}+Q(\lambda)^{2},
$$

where

$$
\begin{gathered}
P(\lambda)=a \lambda^{4}+b \lambda^{3}+c \lambda^{2}+d \lambda+e \\
Q(\lambda)=f \lambda^{4}+g \lambda^{3}+h \lambda^{2}+i \lambda+j \\
a=C_{12}^{2}+C_{34}^{2}, \\
b=2 C_{12} M_{12}+2 C_{34} M_{34}(=0), \\
c=M_{13}^{2}+M_{14}^{2}+M_{23}^{2}+M_{24}^{2}+M_{12}^{2}+M_{34}^{2}+2 C_{12} \Gamma_{12}+2 C_{34} \Gamma_{34}, \\
d=2 \Gamma_{12} M_{12}+2 \Gamma_{13} M_{13}+2 \Gamma_{14} M_{14}+2 \Gamma_{23} M_{23}+2 \Gamma_{24} M_{24}+2 \Gamma_{34} M_{34} \\
e=\Gamma_{12}^{2}+\Gamma_{13}^{2}+\Gamma_{14}^{2}+\Gamma_{23}^{2}+\Gamma_{24}^{2}+\Gamma_{34}^{2}, \\
f=C_{12} C_{34} \\
g=C_{12} M_{34}+C_{34} M_{12}(=0), \\
h=\Gamma_{34} C_{12}+\Gamma_{12} C_{34}+M_{12} M_{34}+M_{23} M_{14}-M_{13} M_{24}, \\
i=M_{34} \Gamma_{12}+M_{12} \Gamma_{34}+M_{14} \Gamma_{23}+M_{23} \Gamma_{14}-\Gamma_{13} M_{24}-\Gamma_{24} M_{13}, \\
j=\Gamma_{34} \Gamma_{12}+\Gamma_{23} \Gamma_{14}-\Gamma_{13} \Gamma_{24} .
\end{gathered}
$$

In the standard Poisson structure on the semidirect product $s o(4) \times s o(4)$ the functions $d, e, i, j$ are Casimir functions, $c, h$ are first integrals, and $b=0, g=0$ are the invariant relations. As we already mentioned general orbits of co-adjoint action are eight-dimensional, thus for complete integrability one needs four independent integrals in involution.

\subsection{The $n$-Dimensional Hess-Appel'rot Systems}

In Dragović and Gajić (2006) we introduced also Hess-Appel'rot systems of arbitrary dimension.

Definition 4.3 The $n$-dimensional Hess-Appel'rot system is described by the Eq. (4.3), and satisfies the conditions: 
1.

$$
\Omega=J M+M J, \quad J=\left(\begin{array}{ccccccc}
J_{1} & 0 & J_{13} & 0 & 0 & \ldots & 0 \\
0 & J_{1} & 0 & J_{24} & 0 & \ldots & 0 \\
J_{13} & 0 & J_{3} & 0 & 0 & \ldots & 0 \\
0 & J_{24} & 0 & J_{3} & 0 & \ldots & 0 \\
0 & 0 & 0 & 0 & 0 & \ldots & 0 \\
. & . & . & . & \ldots & \ldots & . \\
. & . & . & . & \ldots & . \\
0 & 0 & 0 & 0 & 0 & \ldots & J_{3}
\end{array}\right),
$$

2.

$$
\chi=\left(\begin{array}{ccccc}
0 & \chi_{12} & 0 & \ldots & 0 \\
-\chi_{12} & 0 & 0 & \ldots & 0 \\
0 & 0 & 0 & \ldots & 0 \\
0 & 0 & 0 & \ldots & 0 \\
. & . & \ldots & . \\
. & . & \ldots & \ldots & 0 \\
0 & 0 & 0 & \ldots & 0
\end{array}\right)
$$

Direct calculations give the following lemma:

Lemma 4.3 (Dragović and Gajić 2006) For the n-dimensional Hess-Appel'rot system, the following relations are satisfied:

1.

$$
\begin{aligned}
\dot{M}_{12}= & J_{13}\left(M_{12} M_{13}+M_{24} M_{34}+\sum_{p=5}^{n} M_{2 p} M_{3 p}\right) \\
& +J_{24}\left(M_{12} M_{24}+M_{13} M_{34}-\sum_{p=5}^{n} M_{1 p} M_{4 p}\right) \\
\dot{M}_{34}= & -J_{13}\left(M_{13} M_{34}+M_{24} M_{12}+\sum_{p=5}^{n} M_{1 p} M_{p 4}\right) \\
& -J_{24}\left(M_{13} M_{12}+M_{24} M_{34}+\sum_{p=5}^{n} M_{2 p} M_{3 p}\right), \\
\dot{M}_{3 p}= & -J_{13}\left(M_{13} M_{3 p}+M_{2 p} M_{12}\right)-J_{24}\left(M_{34} M_{2 p}+M_{23} M_{4 p}\right) \\
& +M_{34} \Omega_{4 p}-\Omega_{34} M_{4 p}+\sum_{k=5}^{n}\left(M_{3 k} \Omega_{k p}-\Omega_{3 k} M_{4 p}\right), p>4,
\end{aligned}
$$




$$
\begin{aligned}
\dot{M}_{4 p}= & J_{13}\left(-M_{14} M_{3 p}+M_{1 p} M_{34}\right)+J_{24}\left(M_{12} M_{1 p}-M_{24} M_{4 p}\right) \\
& -M_{34} \Omega_{3 p}+\Omega_{34} M_{3 p}+\sum_{k=5}^{n}\left(M_{4 k} \Omega_{k p}-\Omega_{4 k} M_{4 p}\right), p>4,
\end{aligned}
$$

2. $\dot{M}_{k l}=0, k, l>4$.

3. The n-dimensional Hess-Appel'rot case has the following system of invariant relations

$$
M_{12}=0, \quad M_{l p}=0, \quad l, p \geq 3 .
$$

By diagonalizing the matrix $J$, we come to another definition

Definition 4.4 (Dragović and Gajić 2006) The $n$-dimensional Hess-Appel'rot system is described by the equations (4.3), and satisfies the conditions

1. $\Omega=\tilde{J} M+M \tilde{J}, \quad \tilde{J}=\operatorname{diag}\left(\tilde{J}_{1}, \tilde{J}_{2}, \tilde{J}_{3}, \tilde{J}_{4}, \ldots, \tilde{J}_{4}\right)$,

2.

$$
\tilde{\chi}=\left(\begin{array}{cccccc}
0 & \tilde{\chi}_{12} & 0 & \tilde{\chi}_{14} & \ldots & 0 \\
-\tilde{\chi}_{12} & 0 & \tilde{\chi}_{23} & 0 & \ldots & 0 \\
0 & -\tilde{\chi}_{23} & 0 & \tilde{\chi}_{34} & \ldots & 0 \\
-\tilde{\chi}_{14} & 0 & -\tilde{\chi}_{34} & 0 & \ldots & 0 \\
. & . & . & . & \ldots & . \\
. & . & . & . & \ldots & 0 \\
0 & 0 & 0 & 0 & \ldots & 0
\end{array}\right)
$$

3.

$$
\begin{gathered}
\tilde{J}_{3}-\tilde{J}_{4}=\tilde{J}_{2}-\tilde{J}_{1}, \\
\frac{\tilde{J}_{3}-\tilde{J}_{1}}{\sqrt{1+t_{1}^{2}}}=\frac{\tilde{J}_{4}-\tilde{J}_{2}}{\sqrt{1+t_{2}^{2}}} \\
\tilde{\chi}_{12} \tilde{\chi}_{34}+\tilde{\chi}_{14} \tilde{\chi}_{23}=0
\end{gathered}
$$

where

$$
\begin{aligned}
t_{1} & :=\frac{2\left(\tilde{\chi}_{14} \tilde{\chi}_{34}-\tilde{\chi}_{12} \tilde{\chi}_{23}\right)}{\tilde{\chi}_{14}^{2}-\tilde{\chi}_{34}^{2}+\tilde{\chi}_{12}^{2}-\tilde{\chi}_{23}^{2}}, \\
t_{2} & :=\frac{2\left(\tilde{\chi}_{14} \tilde{\chi}_{12}-\tilde{\chi}_{23} \tilde{\chi}_{34}\right)}{-\tilde{\chi}_{14}^{2}-\tilde{\chi}_{34}^{2}+\tilde{\chi}_{12}^{2}+\tilde{\chi}_{23}^{2}} .
\end{aligned}
$$

As in the dimension four, there is an equivalence of the definitions. 
Proposition 4.4 (Dragović and Gajić 2006) There exists a bi-correspondence between sets of data from Definitions 4.3 and 4.4 .

The following theorem gives a Lax pair for the $n$-dimensional Hess-Appel'rot system.

Theorem 4.2 (Dragović and Gajić 2006) The n-dimensional Hess-Appel'rot system has the following Lax pair

$$
\begin{aligned}
& \dot{L}(\lambda)=[L(\lambda), A(\lambda)], \\
& L(\lambda)=\lambda^{2} C+\lambda M+\Gamma, \quad A(\lambda)=\lambda \chi+\Omega, \quad C=\frac{1}{J_{1}+J_{3}} \chi .
\end{aligned}
$$

Remark 4.2 Jovanović (2007) introduced another higher-dimensional generalizations of the Hess-Appel'rot system, one on $e(n)$, using partial Lagrange-Routh reduction (see also Dragović et al. 2009). These systems can be also seen as perturbation of the Lagrange tops, this time of the Lagrange tops defined by Belyaev (4.2). These Hess-Appel'rot systems are defined by

$$
J=\operatorname{diag}\left(J_{1}, J_{1}, \ldots, J_{1}, J_{n}\right)+J_{1 n}\left(E_{1} \otimes E_{n}+E_{n} \otimes E_{1}\right), \quad X=\left(0, \ldots 0, x_{n}\right)
$$

The invariant relations of these $n$-dimensional Hess-Appel'rot systems are:

$$
M_{i j}=0, \quad 1 \leq i<j \leq n-1
$$

\subsection{Classical Integration of the Four-Dimensional Hess-Appel'rot System}

Detailed classical and algebro-geometric integration procedures for the four-dimensional Hess-Appel'rot case are presented in Dragović and Gajić (2006). Here again Eq. (4.7) are useful for classical integration. We have:

$$
\chi_{1}=\left(0,0,-\frac{1}{2}\left(\chi_{12}+\chi_{34}\right)\right), \quad \chi_{2}=\left(0,0,-\frac{1}{2}\left(\chi_{12}-\chi_{34}\right)\right) .
$$

Integrals of the motion are

$$
\begin{aligned}
\left\langle M_{i}, M_{i}\right\rangle+2 \frac{1}{J_{1}+J_{3}}\left\langle\chi_{i}, \Gamma_{i}\right\rangle & =h_{i}, \\
\left\langle\Gamma_{i}, \Gamma_{i}\right\rangle & =1, \quad i=1,2, \\
\left\langle M_{i}, \Gamma_{i}\right\rangle & =c_{i}, \\
\left\langle\chi_{i}, M_{i}\right\rangle & =0 .
\end{aligned}
$$

Here the metric that gives connections between $M$ and $\Omega$ is different from that for Lagrange bitop. We have 


$$
\begin{aligned}
\Omega_{1}= & \left(\left(J_{1}+J_{3}\right) M_{(1) 1}-\left(J_{13}-J_{24}\right) M_{(2) 3},\left(J_{1}+J_{3}\right) M_{(1) 2},\right. \\
& \left.\left(J_{1}+J_{3}\right) M_{(1) 3}+\left(J_{1}-J_{3}\right) M_{(2) 3}-\left(J_{13}+J_{24}\right) M_{(2) 1}\right), \\
\Omega_{2}= & \left(\left(J_{1}+J_{3}\right) M_{(2) 1}-\left(J_{13}+J_{24}\right) M_{(1) 3},\left(J_{1}+J_{3}\right) M_{(2) 2},\right. \\
& \left.\left(J_{1}+J_{3}\right) M_{(2) 3}+\left(J_{1}-J_{3}\right) M_{(1) 3}-\left(J_{13}-J_{24}\right) M_{(1) 1}\right),
\end{aligned}
$$

where again $M_{(i) j}$ is the $j$-th component of the vector $M_{i}$. Using these expressions, Eq. (4.7) can be rewritten in the following form:

$$
\begin{aligned}
\dot{M}_{(1) 1}= & 2\left[\left(J_{1}-J_{3}\right) M_{(1) 2} M_{(2) 3}-\left(J_{13}+J_{24}\right) M_{(1) 2} M_{(2) 1}+\Gamma_{(1) 2} \chi(1) 3\right] \\
\dot{M}_{(1) 2}= & 2\left[-\left(J_{1}-J_{3}\right) M_{(2) 3} M_{(1) 1}-\left(J_{13}-J_{24}\right) M_{(1) 3} M_{(2) 3}\right. \\
& \left.+\left(J_{13}+J_{24}\right) M_{(1) 1} M_{(2) 1}-\Gamma_{(1) 1} \chi_{(1) 3}\right], \\
\dot{M}_{(1) 3}= & 2\left(J_{13}-J_{24}\right) M_{(1) 2} M_{(2) 3}, \\
\dot{\Gamma}_{(1) 1}= & 2\left[\Gamma_{(1) 2}\left(\left(J_{1}+J_{3}\right) M_{(1) 3}+\left(J_{1}-J_{3}\right) M_{(2) 3}-\left(J_{13}+J_{24}\right) M_{(2) 1}\right)\right. \\
& \left.-\Gamma_{(1) 3}\left(J_{1}+J_{3}\right) M_{(1) 2}\right], \\
\dot{\Gamma}_{(1) 2}= & 2\left[\Gamma_{(1) 3}\left(\left(J_{1}+J_{3}\right) M_{(1) 1}-\left(J_{13}-J_{24}\right) M_{(2) 3}\right)\right. \\
& \left.-\Gamma_{(1) 1}\left(\left(J_{1}+J_{3}\right) M_{(1) 3}+\left(J_{1}-J_{3}\right) M_{(2) 3}-\left(J_{13}+J_{24}\right) M_{(2) 1}\right)\right], \\
\dot{\Gamma}_{(1) 3}= & 2\left[\Gamma_{(1) 1}\left(J_{1}+J_{3}\right) M_{(1) 2}-\Gamma_{(1) 2}\left(\left(J_{1}+J_{3}\right) M_{(1) 1}-\left(J_{13}-J_{24}\right) M_{(2) 3}\right)\right],
\end{aligned}
$$

and

$$
\begin{aligned}
\dot{M}_{(2) 1}= & 2\left[\left(J_{1}-J_{3}\right) M_{(2) 2} M_{(1) 3}-\left(J_{13}-J_{24}\right) M_{(2) 2} M_{(1) 1}+\Gamma_{(2) 2} \chi_{(2) 3}\right], \\
\dot{M}_{(2) 2}= & 2\left[-\left(J_{1}-J_{3}\right) M_{(1) 3} M_{(2) 1}-\left(J_{13}+J_{24}\right) M_{(2) 3} M_{(1) 3}+\right. \\
& \left.\left(J_{13}-J_{24}\right) M_{(2) 1} M_{(1) 1}-\Gamma_{(2) 1} \chi(2) 3\right] \\
\dot{M}_{(2) 3}= & 2\left(J_{13}+J_{24}\right) M_{(2) 2} M_{(1) 3}, \\
\dot{\Gamma}_{(2) 1}= & 2\left[\Gamma_{(2) 2}\left(\left(J_{1}+J_{3}\right) M_{(2) 3}+\left(J_{1}-J_{3}\right) M_{(1) 3}-\left(J_{13}-J_{24}\right) M_{(1) 1}\right)-\right. \\
& \left.\Gamma_{(2) 3}\left(J_{1}+J_{3}\right) M_{(2) 2}\right] \\
\dot{\Gamma}_{(2) 2}= & 2\left[\Gamma_{(2) 3}\left(\left(J_{1}+J_{3}\right) M_{(2) 1}-\left(J_{13}+J_{24}\right) M_{(1) 3}\right)-\right. \\
& \left.\Gamma_{(2) 1}\left(\left(J_{1}+J_{3}\right) M_{(2) 3}+\left(J_{1}-J_{3}\right) M_{(1) 3}-\left(J_{13}-J_{24}\right) M_{(1) 1}\right)\right], \\
\dot{\Gamma}_{(2) 3}= & 2\left[\Gamma_{(2) 1}\left(J_{1}+J_{3}\right) M_{(2) 2}-\Gamma_{(2) 2}\left(\left(J_{1}+J_{3}\right) M_{(2) 1}-\left(J_{13}+J_{24}\right) M_{(1) 3}\right)\right] .
\end{aligned}
$$

One can see here that $M_{(1) 3}=M_{(2) 3}=0$, giving two invariant relations introduced before.

Let us introduce coordinates $K_{i}$ and $l_{i}$ as follows:

$$
M_{(i) 1}=K_{i} \sin l_{i}, \quad M_{(i) 2}=K_{i} \cos l_{i}, \quad i=1,2 .
$$


From Eqs. (4.17), (4.18), using integrals (4.16), we have

$$
\dot{\Gamma}_{(1) 3}^{2}=4\left(J_{1}+J_{3}\right)^{2}\left[\left(1-\Gamma_{(1) 3}^{2}\right)\left(h_{1}-\frac{2}{J_{1}+J_{3}} \chi_{(1) 3} \Gamma_{(1) 3}\right)-c_{1}^{2}\right]=P_{3}\left(\Gamma_{(1) 3}\right) .
$$

Thus $\Gamma_{(1) 3}$ can be solved by an elliptic quadrature. Also from the energy integral we have that

$$
K_{1}^{2}=h_{1}-\frac{2}{J_{1}+J_{3}} \chi_{(1) 3} \Gamma_{(1) 3}
$$

Since $\tan l_{1}=\frac{M_{(1) 1}}{M_{(1) 2}}$, we have:

$$
i_{1}=-2\left(J_{13}+J_{24}\right) K_{2} \sin l_{2}+\frac{2 \chi(1) 3 c_{1}}{K_{1}^{2}}
$$

and

$$
K_{1}^{2} \Gamma_{(1) 2}^{2}-2 c_{1} M_{(1) 2} \Gamma_{(1) 2}+c_{1}^{2}-M_{(1) 1}^{2}\left(1-\Gamma_{(1) 3}^{2}\right)=0 .
$$

Similarly, one gets:

$$
\begin{aligned}
& \dot{\Gamma}_{(2) 3}^{2}=4\left(J_{1}+J_{3}\right)^{2}\left[\left(1-\Gamma_{(2) 3}^{2}\right)\left(h_{2}-\frac{2}{J_{1}+J_{3}} \chi_{(2) 3} \Gamma_{(2) 3}\right)-c_{2}^{2}\right]=P_{3}\left(\Gamma_{(2) 3}\right), \\
& K_{2}^{2}= h_{2}-\frac{2}{J_{1}+J_{3}} \chi_{(2) 3} \Gamma_{(2) 3} \\
& \dot{l}_{2}=-2\left(J_{13}-J_{24}\right) K_{1} \sin l_{1}+\frac{2 \chi_{(2) 3} c_{2}}{K_{2}^{2}} \\
& K_{2}^{2} \Gamma_{(2) 2}^{2}-2 c_{2} M_{(2) 2} \Gamma_{(2) 2}+c_{2}^{2}-M_{(2) 1}^{2}\left(1-\Gamma_{(2) 3}^{2}\right)=0
\end{aligned}
$$

From the previous considerations one concludes that integration of the fourdimensional Hess-Appel'rot system leads to a system of two differential equations (for $l_{1}$ and $l_{2}$ ) of the first order and two elliptic integrals, associated with elliptic curves $E_{1}$ and $E_{2}$ defined by

$$
E_{i}: y^{2}=P_{i}(x)=8 A_{i} x^{3}-4 B_{i} x^{2}-8 A_{i} x-4 C_{i}, \quad i=1,2
$$

where

$$
A_{i}=\left(J_{1}+J_{3}\right) \chi_{(i) 3}, \quad B_{i}=\left(J_{1}+J_{3}\right)^{2} h_{i}, \quad C_{i}=\left(J_{1}+J_{3}\right)^{2}\left(c_{i}^{2}-h_{i}\right) .
$$

This is a typical situation for the Hess-Appel'rot systems that additional integrations are required.

In Dragović and Gajić (2006) the algebro-geometric integration procedure is presented. It is closely related to the integration of the Lagrange bitop. 


\section{Four-Dimensional Grioli-Type Precessions}

In this Section we review recent results on higher-dimensional precessions, from Dragović and Gajić (2014).

A regular precession of a motion of a heavy rigid body fixed at a point is characterized by the existence of two distinguished axes, one fixed in the body, and another one fixed in the space, forming the angle constant in time. This condition has an equivalent formulation in terms of the existence of a decomposition of the angular velocity vector into two components: the first component is fixed in the body and the second component is fixed in the space. A famous example is provided by the Lagrange top admitting such a motion as the rotation about the vertical axis, which is the direction of the gravitational force. However, the Lagrange top is symmetric and the question was whether such a motion can be realized by a nonsymmetric body. In 1947, Grioli presented the conditions on the moments of inertia and the radius vector of the center of masses, such that an asymmetric rigid body admits nonvertical regular precessions (Grioli 1947). These conditions imposed the center of masses to lie on the line through the fixed point perpendicular to the circular section of the ellipsoid of inertia. Zhukovskii formulated a similar condition in his characterization of the classical Hess-Appel'rot case, presented in Sect. 2.5. Thus, the Grioli case is dual to the Hess-Appel'rot case.

In this Section, following Dragović and Gajić (2014), we discuss the existence of non-vertical precessions of non-symmetric rigid bodies in four-dimensional case.

The classical Grioli case can be considered as a certain perturbation of the Lagrange top. The Grioli solution can be obtained starting from a particular solution of the motion of the Lagrange top, the one that presents the vertical regular precession. Using that as a staring point, we consider the four-dimensional Lagrange top on so(4) defined by Belyaev (1981). We construct its particular solution, which is the four-dimensional analogue of the vertical precession. Starting from this solution, we construct a four dimensional analogue of the Grioli case - the case of a four-dimensional nonvertical regular precession by a nonsymmetric body. In order to justify the use of the notion of precession in this four dimensional case, we show that the angular velocity is decomposed into two components: one which represents a rotation of a three-dimensional subspace in $\mathbb{R}^{4}$ that is fixed in the space, and another one which represents a rotation of a three-dimensional subspace fixed in the body. The angle between the corresponding vectors orthogonal to these two three-dimensional spaces is constant in time. In this way, an analogy between the four-dimensional precessions and the three-dimensional ones is established.

\subsection{The Classical Grioli Case}

By the above definition, a regular precession of three-dimensional rigid body motion assumes the existence of two vectors: a vector $u$ fixed in the space and a vector $U$ fixed in the body, such that the angle between them remains constant during the motion. Suppose the moving frame $O E_{1} E_{2} E_{3}$ and the fixed frame $O e_{1} e_{2} e_{3}$ are chosen in a way that $U=E_{3}$ and $u=e_{3}$. Then the nutation angle $\theta$ is constant. Grioli proved in 
Grioli (1947) that a regular precession is possible if $\theta=\pi / 2$ and $\dot{\psi}=\dot{\varphi}=c=$ const. Expressed through the Euler angles $\psi, \varphi, \theta$, the angular velocity $\Omega$ and the vector $u$ in the moving coordinate system are:

$$
\begin{aligned}
\Omega & =(c \sin (c t), c \cos (c t), c), \\
u & =(\sin (c t), \cos (c t), 0)
\end{aligned}
$$

The unit vertical vector $\Gamma$ is given by

$$
\begin{gathered}
\Gamma=\left(\cos \sigma \sin (c t)+\sin \sigma \cos ^{2}(c t)\right. \\
\cos \sigma \cos (c t)-\sin \sigma \cos (c t) \sin (c t), \sin \sigma \sin (c t))
\end{gathered}
$$

where $\sigma$ is constant (see Rubanovskii 1985; Grioli 1947; Borisov and Mamaev 2001).

Grioli obtained the conditions for a nonvertical regular precession by plugging the last expressions into the Euler-Poisson equations (2.2):

$$
I=\left(\begin{array}{ccc}
I_{1} & 0 & I_{13} \\
0 & I_{1} & 0 \\
I_{13} & 0 & I_{3}
\end{array}\right), \quad \chi=\left(0,0, Z_{0}\right),
$$

where $I_{3} c^{2}=Z_{0} \cos \sigma, I_{13} c^{2}=-Z_{0} \sin \sigma$.

If $\Gamma=u$ then $I_{13}=0$, presenting the case of the regular vertical precession of the Lagrange top. Here $\Omega$ and $\Gamma$ are:

$$
\begin{aligned}
\Omega & =(c \sin (c t), c \cos (c t), c), \\
\Gamma & =(\sin (c t), \cos (c t), 0) .
\end{aligned}
$$

Thus, we see the Grioli case as a certain perturbation of the Lagrange top.

On the other hand, the Grioli precession can be obtained starting from the particular solutions (5.2) of the Lagrange top. By plugging the solutions for $\Omega$ from (5.2) into the Euler-Poisson Eq. (2.2) and using the conditions (5.1), from the first two equations, one determines $\Gamma_{1}$ and $\Gamma_{2}$. Then, one gets the expression for $\Gamma_{3}$ from the differential equation for $\Gamma_{1}$. Quite miraculously, the remaining differential equations are identically satisfied then.

In the next Section we will construct a particular solution of the four-dimensional Lagrange top first. Then, by using the pattern described above, we will construct a four-dimensional analogue of the Grioli precession.

Remark 5.1 In a basis in which the matrix $I$ has a diagonal form $I=\operatorname{diag}\left(I_{1}, I_{2}, I_{3}\right)$, the Grioli conditions can be rewritten as:

$$
\chi=\left(X_{0}, 0, Z_{0}\right), \quad \sqrt{I_{2}-I_{3}} X_{0} \pm \sqrt{I_{1}-I_{2}} Z_{0}=0
$$

As we have already mentioned, the geometric interpretation of these conditions is that the center of masses lies on the line through the fixed point perpendicular to the 
circular section of the ellipsoid of inertia. If instead of $I_{i}$ one takes their inverses, the same conditions define the classical Hess-Appel'rot case of motion of a heavy rigid body. The ellipsoid of inertia is replaced by the gyroscopic ellipsoid, and one comes to the Zhukovski geometric interpretation of the Hess-Appel'rot case. In that sense we may observe that the Grioli and the Hess-Appel'rot cases are dual to each other.

An analogue of the Grioli precession for a motion of a rigid body in an ideal incompressible fluid is given by Rubanovskii (1985).

\subsection{Four-Dimensional Grioli Case}

For $n=4$ the Lagrange top on $e(4)$ defined by Belyaev (4.2) is given by: $I=$ $\operatorname{diag}\left(I_{1}, I_{1}, I_{1}, I_{4}\right)$ and $X=\left(0,0,0, x_{4}\right)$. Thus, the Eq. (4.1) become:

$$
\begin{aligned}
\dot{\Omega}_{12} & =0, \quad \dot{\Omega}_{13}=0, \quad \dot{\Omega}_{23}=0, \\
\left(I_{1}+I_{4}\right) \dot{\Omega}_{14} & =\left(I_{1}-I_{4}\right)\left(\Omega_{12} \Omega_{24}+\Omega_{13} \Omega_{34}\right)+x_{4} \Gamma_{1}, \\
\left(I_{1}+I_{4}\right) \dot{\Omega}_{24} & =\left(I_{1}-I_{4}\right)\left(-\Omega_{12} \Omega_{14}+\Omega_{23} \Omega_{34}\right)+x_{4} \Gamma_{2}, \\
\left(I_{1}+I_{4}\right) \dot{\Omega}_{34} & =-\left(I_{1}-I_{4}\right)\left(\Omega_{13} \Omega_{14}+\Omega_{23} \Omega_{24}\right)+x_{4} \Gamma_{3}, \\
\dot{\Gamma}_{1} & =-\Omega_{12} \Gamma_{2}-\Omega_{13} \Gamma_{3}-\Omega_{14} \Gamma_{4}, \\
\dot{\Gamma}_{2} & =-\Omega_{21} \Gamma_{1}-\Omega_{23} \Gamma_{3}-\Omega_{24} \Gamma_{4}, \\
\dot{\Gamma}_{3} & =-\Omega_{31} \Gamma_{1}-\Omega_{32} \Gamma_{2}-\Omega_{34} \Gamma_{4}, \\
\dot{\Gamma}_{4} & =-\Omega_{41} \Gamma_{1}-\Omega_{42} \Gamma_{2}-\Omega_{43} \Gamma_{3} .
\end{aligned}
$$

To construct a particular solution of Eq. (5.3), let us start with the case when $x_{4}=0$. This is the symmetric Euler case. Let us fix the values of the first three linear integrals $\Omega_{12}=-a, \Omega_{13}=-b, \Omega_{23}=-c$. Then the second three equations of (5.3) are of the form:

$$
\left(\begin{array}{l}
\dot{x} \\
\dot{y} \\
\dot{z}
\end{array}\right)=\left(\begin{array}{lll}
0 & -a & -b \\
a & 0 & -c \\
b & c & 0
\end{array}\right)\left(\begin{array}{l}
x \\
y \\
z
\end{array}\right) .
$$

The general solution of this system is given by:

$$
\begin{aligned}
x= & -c C_{1}+(-a c \cos (\lambda t)-b \lambda \sin (\lambda t)) C_{2} \\
& +(-a c \sin (\lambda t)+b \lambda \cos (\lambda t)) C_{3}, \\
y= & b C_{1}+(a b \cos (\lambda t)-c \lambda \sin (\lambda t)) C_{2} \\
& +(a b \sin (\lambda t)+c \lambda \cos (\lambda t)) C_{3}, \\
z= & -a C_{1}+\left(b^{2}+c^{2}\right) \cos (\lambda t) C_{2}+\left(b^{2}+c^{2}\right) \sin (\lambda t) C_{3},
\end{aligned}
$$

where $\lambda=\sqrt{a^{2}+b^{2}+c^{2}}$. Let us assume that $\Gamma_{i}$ are proportional to $\dot{\Omega}_{i 4}$ for $i=$ $1,2,3$. Then using (5.4), and choosing $C_{1}=C_{3}=0, C_{2}=1$, one gets a particular 
solution for $\Omega_{14}, \Omega_{24}, \Omega_{34}$ and $\Gamma_{1}, \Gamma_{2}, \Gamma_{3}$ for the four-dimensional Lagrange case. Thus, we have proved the following statement:

Proposition 5.1 (Dragović and Gajić 2014) When $x_{4}=-2 \lambda^{3} I_{1}$ the Eq. (5.3) of motion of the four-dimensional Lagrange top have a particular solution:

$$
\begin{aligned}
\Omega_{12} & =-a, \Omega_{13}=-b, \Omega_{23}=-c, \\
\Omega_{14} & =a c \cos (\lambda t)-b \lambda \sin (\lambda t), \\
\Omega_{24} & =-a b \cos (\lambda t)-c \lambda \sin (\lambda t), \\
\Omega_{34} & =-\left(b^{2}+c^{2}\right) \cos (\lambda t), \\
\Gamma_{1} & =\frac{1}{\lambda^{2}}(a c \sin (\lambda t)+b \lambda \cos (\lambda t)) \\
\Gamma_{2} & =\frac{1}{\lambda^{2}}(-a b \sin (\lambda t)+c \lambda \cos (\lambda t)) \\
\Gamma_{3} & =-\frac{1}{\lambda^{2}}\left(b^{2}+c^{2}\right) \sin (\lambda t) \\
\Gamma_{4} & =0 .
\end{aligned}
$$

It is important to observe that the angular velocity is decomposed into two components: $\Omega=\Omega_{1}+\Omega_{2}$. The first component $\Omega_{1}=\Omega_{12} E_{1} \wedge E_{2}+\Omega_{13} E_{1} \wedge E_{3}+$ $\Omega_{23} E_{2} \wedge E_{3}$ represents a rotation in the three-dimensional space generated by the unit vectors $E_{1}, E_{2}$ and $E_{3}$ and it is constant in the body.

The remaining component, $\Omega_{2}=\Omega_{14} E_{1} \wedge E_{4}+\Omega_{24} E_{2} \wedge E_{4}+\Omega_{34} E_{3} \wedge E_{4}$ can be written more explicitly as:

$\Omega_{2}=\left(\begin{array}{cccc}0 & 0 & 0 & a c \cos (\lambda t)-b \lambda \sin (\lambda t) \\ 0 & 0 & 0 & -a b \cos (\lambda t)-c \lambda \sin (\lambda t) \\ 0 & 0 & 0 & -\left(b^{2}+c^{2}\right) \cos (\lambda t) \\ -a c \cos (\lambda t) & a b \cos (\lambda t) & \left(b^{2}+c^{2}\right) \cos (\lambda t) & 0 \\ +b \lambda \sin (\lambda t) & +c \lambda \sin (\lambda t) & \end{array}\right)$

Thus, $\Omega_{2}$ satisfies the Poisson equations

$$
\dot{\Omega}_{2}=\left[\Omega_{2}, \Omega\right] \text {. }
$$

Consequently, $\Omega_{2}$ represents a rotation of three-dimensional subspace fixed in the space. One can check that $\Gamma$ is orthogonal to it. Note also that the vector $\Gamma$ is orthogonal to the vector $E_{4}$, so, as in three-dimensional case, we have here a case of the vertical precession.

Proposition 5.2 The particular solution (5.5) of the four-dimensional Lagrange top represents a four-dimensional vertical precession.

In the previous subsection, we explained the procedure how to construct the classical Grioli precession in three-dimensional case, starting from the vertical precession of the 
Lagrange top. Let us apply here the same pattern to get a four-dimensional analogue of the Grioli case.

Denote by $u$ a non-vertical vector fixed in the space. We are looking for a motion such that there exists a vector $U$ fixed in the body, with the property that the angle between $u$ and $U$ is constant in time. Let us, by analogy with the three-dimensional case, fix a moving frame such with $E_{4}=U$ and a fixed frame having $e_{4}=u$. Then, the matrix $I$ is not necessary diagonal any more.

Definition 5.1 (Dragović and Gajić 2014) The four-dimensional Grioli case of rigid body motion is described by the Eq. (4.1) with

$$
I=\left(\begin{array}{cccc}
I_{1} & 0 & 0 & I_{14} \\
0 & I_{1} & 0 & 0 \\
0 & 0 & I_{1} & 0 \\
I_{14} & 0 & 0 & I_{4}
\end{array}\right)
$$

and $X=\left(0,0,0, x_{4}\right)$.

More explicitly, the equations of motion (4.1) are:

$$
\begin{aligned}
2 I_{1} \dot{\Omega}_{12}-I_{14} \dot{\Omega}_{24}= & I_{14}\left(\Omega_{23} \Omega_{34}-\Omega_{12} \Omega_{14}\right), \\
2 I_{1} \dot{\Omega}_{13}-I_{14} \dot{\Omega}_{34}= & -I_{14}\left(\Omega_{23} \Omega_{24}+\Omega_{13} \Omega_{14}\right), \\
\dot{\Omega}_{23}= & 0, \\
\left(I_{1}+I_{4}\right) \dot{\Omega}_{14}= & \left(I_{1}-I_{4}\right)\left(\Omega_{12} \Omega_{24}+\Omega_{13} \Omega_{34}\right) \\
& +I_{14}\left(\Omega_{12}^{2}+\Omega_{13}^{2}-\Omega_{24}^{2}-\Omega_{34}^{2}\right)+x_{4} \Gamma_{1}, \\
\left(I_{1}+I_{4}\right) \dot{\Omega}_{24}-I_{14} \dot{\Omega}_{12}= & \left(I_{1}-I_{4}\right)\left(-\Omega_{12} \Omega_{14}+\Omega_{23} \Omega_{34}\right) \\
& +I_{14}\left(\Omega_{24} \Omega_{14}+\Omega_{13} \Omega_{23}\right)+x_{4} \Gamma_{2}, \\
\left(I_{1}+I_{4}\right) \dot{\Omega}_{34}-I_{14} \dot{\Omega}_{13}= & -\left(I_{1}-I_{4}\right)\left(\Omega_{13} \Omega_{14}+\Omega_{23} \Omega_{24}\right) \\
& +I_{14}\left(\Omega_{14} \Omega_{34}-\Omega_{12} \Omega_{23}\right)+x_{4} \Gamma_{3}, \\
\dot{\Gamma}_{1}= & -\Omega_{12} \Gamma_{2}-\Omega_{13} \Gamma_{3}-\Omega_{14} \Gamma_{4}, \\
\dot{\Gamma}_{2}= & -\Omega_{21} \Gamma_{1}-\Omega_{23} \Gamma_{3}-\Omega_{24} \Gamma_{4}, \\
\dot{\Gamma}_{3}= & -\Omega_{31} \Gamma_{1}-\Omega_{32} \Gamma_{2}-\Omega_{34} \Gamma_{4}, \\
\dot{\Gamma}_{4}= & -\Omega_{41} \Gamma_{1}-\Omega_{42} \Gamma_{2}-\Omega_{43} \Gamma_{3} .
\end{aligned}
$$

Our next goal is to construct a particular solution of the four-dimensional Grioli case. We start from the particular solution (5.5) of the four-dimensional Lagrange top. Our specific choice of the moving frame with the axis $E_{4}$ coinciding with the direction of the vector $U$, motivates us to assume the particular solution for the $\Omega$-s for the four-dimensional Grioli case to be the same as for the above particular solution of the four-dimensional Lagrange top. By plugging these expressions for $\Omega$-s into the differential equations for $\Omega$ 's from (5.6), one gets the expressions for the $\Gamma_{1}, \Gamma_{2}$, and $\Gamma_{3}$. Then, from one of the three differential equations, say the one for $\Gamma_{1}$, one gets the expression for $\Gamma_{4}$. Finally, one needs to check if the remaining differential equations are identically satisfied. These compatibility conditions are satisfied again 
miraculously, under a very mild constraint on the parameters: if and only if $b^{2}+c^{2}=1$, or equivalently if and only if $\lambda=\sqrt{1+a^{2}}$. Summarizing, we get the following theorem:

Theorem 5.1 (Dragović and Gajić 2014) Assume

$$
b^{2}+c^{2}=1, \quad x_{4}=-2 \lambda^{3} I_{1}\left(1+\frac{I_{14}^{2}}{I_{1}^{2}}\right) .
$$

Then, the four-dimensional Grioli case has the following particular solution:

$$
\begin{aligned}
\Omega_{12}= & -a, \Omega_{13}=-b, \Omega_{23}=-c \\
\Omega_{14}= & a c \cos (\lambda t)-b \lambda \sin (\lambda t), \\
\Omega_{24}= & -a b \cos (\lambda t)-c \lambda \sin (\lambda t), \\
\Omega_{34}= & -\left(b^{2}+c^{2}\right) \cos (\lambda t), \\
\Gamma_{1}= & \frac{I_{1}^{2}}{\lambda^{2}\left(I_{1}^{2}+I_{14}^{2}\right)}\left(a c \sin (\lambda t)+b \lambda \cos (\lambda t)+\frac{I_{14}}{2 \lambda I_{1}}\left(a^{2} c^{2}\left(\cos ^{2}(\lambda t)-\sin ^{2}(\lambda t)\right)\right.\right. \\
& \left.\left.-2 c^{2}+c^{2} \cos ^{2}(\lambda t)+\lambda^{2} \sin ^{2}(\lambda t)-2 a b c \sin (\lambda t) \cos (\lambda t)\right)\right) \\
\Gamma_{2}= & \frac{I_{1}^{2}}{\lambda^{2}\left(I_{1}^{2}+I_{14}^{2}\right)}\left(-a b \sin (\lambda t)+c \lambda \cos (\lambda t)+\frac{I_{14}}{2 \lambda I_{1}}\left(\lambda^{2} b c+b c \sin ^{2}(\lambda t)\right.\right. \\
& \left.\left.-2 a^{2} b c \cos ^{2}(\lambda t)+a \lambda \sin (\lambda t) \cos (\lambda t)\left(1-2 c^{2}\right)\right)\right) \\
\Gamma_{3}= & \frac{I_{1}^{2}}{\lambda^{2}\left(I_{1}^{2}+I_{14}^{2}\right)}\left(-\left(b^{2}+c^{2}\right) \sin (\lambda t)\right. \\
& \left.+\frac{I_{14}}{2 \lambda I_{1}}\left(-a c-a c \cos ^{2}(\lambda t)+\lambda b \sin (\lambda t) \cos (\lambda t)\right)\right) \\
\Gamma_{4}= & \frac{I_{1} I_{14}}{2 \lambda^{2}\left(I_{1}^{2}+I_{14}^{2}\right)} \\
& \times\left(\frac{a b c \lambda\left(\cos ^{2}(\lambda t)-\sin ^{2}(\lambda t)\right)+\left(a^{2} c^{2}+\left(c^{2}+1\right) \lambda^{2}\right) \sin (\lambda t) \cos (\lambda t)}{a c \cos (\lambda t)-a \sin (\lambda t)}\right)
\end{aligned}
$$

This solution represents a four-dimensional precession.

\section{Motion of a Rigid Body in an Ideal Fluid: The Kirchhoff Equations}

A mechanical system similar to the motion of a heavy rigid body fixed at a point is a motion of a rigid body in an ideal incompressible fluid that is at rest at infinity. The equations of motion were derived by Kirchhoff in 1870 (see Kirchhoff 1874). They can be written in the form

$$
\dot{\vec{M}}=\vec{M} \times \frac{\partial H}{\partial \vec{M}}+\vec{\Gamma} \times \frac{\partial H}{\partial \vec{\Gamma}},
$$




$$
\dot{\vec{\Gamma}}=\vec{\Gamma} \times \frac{\partial H}{\partial \vec{M}}
$$

where the Hamiltonian $H$ is a homogeneous quadratic function in $\vec{M}$ and $\vec{\Gamma}$ given by:

$$
H=\frac{1}{2}\langle A \vec{M}, \vec{M}\rangle+\langle B \vec{M}, \vec{\Gamma}\rangle+\frac{1}{2}\langle C \vec{\Gamma}, \vec{\Gamma}\rangle
$$

Here $\vec{M}$ is the impulsive moment and $\vec{\Gamma}$ is the impulsive force. The matrix $A$ is positivedefinite, the matrices $B$ and $C$ are symmetric. Equation (6.1) are Hamiltonian in the standard Lie-Poisson structure given by (2.4). Hence for complete integrability in the Liouville sense one needs one additional independent integral.

The equations of motion of a heavy rigid body fixed at a point (2.2) can be written in the form (6.1) with $H$ from (2.3) as the Hamiltonian function.

\subsection{Integrable Cases}

We will list the known completely integrable cases (for details see for example Borisov and Mamaev 2001).

The first nontrivial integrable case of equations (6.1) was discovered by Kirchhoff in 1870 (see Kirchhoff 1874). It is defined by the conditions:

- Kirchhoff's case (1870):

$$
A=\operatorname{diag}\left(a_{1}, a_{1}, a_{3}\right), \quad B=\operatorname{diag}\left(b_{1}, b_{1}, b_{3}\right), \quad C=\operatorname{diag}\left(c_{1}, c_{1}, c_{3}\right) .
$$

An additional integral is $F_{4}=M_{3}$. It is analogous to the Lagrange case of motion of a heavy rigid body fixed at a point.

- The first Clebsch case (1871):

$$
A=\operatorname{diag}(a, a, a), \quad B=0,
$$

The additional integral is:

$$
F_{4}=a\langle C \vec{M}, \vec{M}\rangle-\operatorname{det}(C)\left\langle C^{-1} \vec{\Gamma}, \vec{\Gamma}\right\rangle .
$$

- The second Clebsch case (1871):

$$
\begin{gathered}
A=\operatorname{diag}\left(a_{1}, a_{2}, a_{3}\right), \quad B=0, \quad C=\operatorname{diag}\left(c_{1}, c_{2}, c_{3}\right) \\
\frac{c_{2}-c_{3}}{a_{1}}+\frac{c_{3}-c_{1}}{a_{2}}+\frac{c_{1}-c_{2}}{a_{3}}=0 .
\end{gathered}
$$

The conditions (6.2) are equivalent to:

$$
\frac{c_{2}-c_{3}}{a_{1}\left(a_{2}-a_{3}\right)}=\frac{c_{3}-c_{1}}{a_{2}\left(a_{3}-a_{1}\right)}=\frac{c_{1}-c_{2}}{a_{3}\left(a_{1}-a_{2}\right)}=\theta,
$$


where $a_{1}, a_{2}, a_{3}$ are pairwise distinct. The additional integral is:

$$
F_{4}=\theta\langle\vec{M}, \vec{M}\rangle-\langle A \vec{\Gamma}, \vec{\Gamma}\rangle
$$

- The Steklov case (1893):

$$
\begin{aligned}
& A=\operatorname{diag}\left(a_{1}, a_{2}, a_{3}\right), \quad B=\operatorname{diag}\left(\mu a_{2} a_{3}, \mu a_{3} a_{1}, \mu a_{1} a_{2}\right), \\
& C=\operatorname{diag}\left(\mu^{2} a_{1}\left(a_{2}-a_{3}\right)^{2}, \mu^{2} a_{2}\left(a_{3}-a_{1}\right)^{2}, \mu^{2} a_{3}\left(a_{1}-a_{2}\right)^{2}\right)
\end{aligned}
$$

where $\mu$ is a constant. The additional integral is:

$$
\begin{aligned}
F_{4}= & \sum_{j}\left(M_{j}^{2}-2 \mu a_{j} M_{j} \Gamma_{j}\right)+\mu^{2}\left(\left(a_{2}-a_{3}\right)^{2} \Gamma_{1}^{2}\right. \\
& \left.+\left(a_{3}-a_{1}\right)^{2} \Gamma_{2}^{2}+\left(a_{1}-a_{2}\right)^{2} \Gamma_{3}^{2}\right) .
\end{aligned}
$$

- The Lyapunov case (1893):

$$
\begin{aligned}
& A=\operatorname{diag}(1,1,1), \quad B=\operatorname{diag}\left(-2 \mu d_{1},-2 \mu d_{2},-2 \mu d_{3}\right), \\
& C=\operatorname{diag}\left(\mu^{2}\left(d_{2}-d_{3}\right)^{2}, \mu^{2}\left(d_{3}-d_{1}\right)^{2}, \mu^{2}\left(d_{1}-d_{2}\right)^{2}\right)
\end{aligned}
$$

The additional integral is:

$$
\begin{aligned}
F_{4}= & \sum_{j} d_{j} M_{j}^{2}+2 \mu\left(d_{2} d_{3} M_{1} \Gamma_{1}+d_{3} d_{1} M_{2} \Gamma_{2}+d_{1} d_{2} M_{3} \Gamma_{3}\right) \\
& +\mu^{2}\left(d_{1}\left(d_{2}-d_{3}\right)^{2} \Gamma_{1}^{2}+d_{2}\left(d_{3}-d_{1}\right)^{2} \Gamma_{2}^{2}+d_{3}\left(d_{1}-d_{2}\right)^{2} \Gamma_{3}^{2}\right)
\end{aligned}
$$

- The Sokolov case (Sokolov 2001):

$$
\begin{gathered}
a_{1}=a_{2}=1, \quad a_{3}=2, \quad b_{13}=\alpha, \quad b_{23}=\beta, \\
c_{12}=-4 \alpha \beta, \quad c_{11}=4 \beta^{2}, \quad c_{22}=4 \alpha^{2}, \quad c_{33}=-4\left(\alpha^{2}+\beta^{2}\right),
\end{gathered}
$$

and additional integral is

$$
F_{4}=\left(M_{3}-\alpha \Gamma_{1}-\beta \Gamma_{2}\right)^{2} P+Q^{2},
$$

where

$$
\begin{aligned}
P= & \left(\alpha^{2}+\beta^{2}\right)\left(M_{3}+2 \alpha \Gamma_{1}+2 \beta \Gamma_{2}\right)^{2}+\left(\beta M_{1}-\alpha M_{2}\right)^{2} \\
Q= & {\left[\alpha M_{1}+\beta M_{2}+\left(\alpha^{2}+\beta^{2}\right) \Gamma_{3}\right]\left(M_{3}+2 \alpha \Gamma_{1}+2 \beta \Gamma_{2}\right) } \\
& +3\left(\beta M_{1}-\alpha M_{2}\right)\left(\beta \Gamma_{1}-\alpha \Gamma_{2}\right) .
\end{aligned}
$$

Among completely integrable cases, let us mention two more cases: the Chaplygin first case that admits the additional integral when $\langle\vec{M}, \vec{\Gamma}\rangle=0$, and the Chaplygin 
second case that, instead of an additional first integral, possesses an invariant relation and it is analogous to the Hess-Appel'rot case.

- The Chaplygin first case (1902):

$$
A=\operatorname{diag}(a, a, 2 a), \quad B=0, \quad C=\operatorname{diag}(c,-c, 0),
$$

On the symplectic leaf given by $\langle\vec{M}, \vec{\Gamma}\rangle=0$, the equations admit an additional first integral:

$$
F_{4}=\left(M_{1}^{2}-M_{2}^{2}+c \Gamma_{3}^{2}\right)^{2}+4 M_{1}^{2} M_{2}^{2}
$$

- The Chaplygin second case (1897).

Chaplygin's second case has an invariant relation, instead of a fourth first integral. It was defined by Chaplygin in 1897 (see Chaplygin 1976). This system was also considered by Kozlov and Onischenko in Kozlov and Onischenko (1982). It is defined by:

$$
\begin{aligned}
A= & \operatorname{diag}\left(a_{1}, a_{2}, a_{3}\right) \\
& b_{13} \sqrt{a_{2}-a_{1}} \mp\left(b_{2}-b_{1}\right) \sqrt{a_{3}-a_{2}}=0, b_{12}=0 \\
& b_{13} \sqrt{a_{3}-a_{2}} \pm\left(b_{3}-b_{2}\right) \sqrt{a_{2}-a_{1}}=0, b_{23}=0 \\
& c_{13} \sqrt{a_{2}-a_{1}} \mp\left(c_{2}-c_{1}\right) \sqrt{a_{3}-a_{2}}=0, c_{12}=0 \\
& c_{13} \sqrt{a_{3}-a_{2}} \pm\left(c_{3}-c_{2}\right) \sqrt{a_{2}-a_{1}}=0, c_{23}=0 .
\end{aligned}
$$

An invariant relation is: $F_{4}=M_{1} \sqrt{a_{2}-a_{1}} \mp M_{3} \sqrt{a_{3}-a_{2}}=0$.

One can easily observe a lot of similarities between the Kirchhoff and the EulerPoisson equations and a parallelism between corresponding integrable cases. However, the problem of integrability for the Kirchhoff equations appeared to be much complicated. Some partial results of the nonexistence of an additional integral were given by Kozlov and Onischenko (1982), Borisov (1996), Barkin and Borisov (1989), Sadetov (2000). The problem of classification of completely integrable cases is still open for the Kirchhoff equations.

\subsection{Three-Dimensional Chaplygin's Second Case}

Conditions (6.3) may be regarded as analogy of the Hess-Appel'rot conditions in the case of motion of a heavy rigid body fixed at a point. We have shown that HessAppel'rot case can be considered as a perturbation of the Lagrange top.

Similarly, the Chaplygin case is a perturbation of the Kirchhoff case. If one chooses the basis where $a_{1}=a_{2}$, the Chaplygin conditions become (see for example Dragović and Gajić 2012; Borisov and Mamaev 2001):

$$
a_{1}=a_{2}, \quad a_{13} \neq 0, \quad B=\operatorname{diag}\left(b_{1}, b_{1}, b_{3}\right), \quad C=\operatorname{diag}\left(c_{1}, c_{1}, c_{3}\right)
$$


The Hamiltonian becomes:

$$
\begin{aligned}
2 H= & a_{1}\left(M_{1}^{2}+M_{2}^{2}\right)+a_{3} M_{3}+2 a_{13} M_{1} M_{3} \\
& +2 b_{1}\left(M_{1} p_{1}+M_{2} p_{2}\right)+2 b_{3} M_{3} p_{3}+c_{1}\left(p_{1}^{2}+p_{2}^{2}\right)+c_{3} p_{3}^{2} \\
= & H_{K}+2 a_{13} M_{1} M_{3} .
\end{aligned}
$$

Here $H_{K}$ is the Hamiltonian for the Kirchhoff case. In the new coordinates the invariant relation is $M_{3}=0$.

\subsubsection{Classical Integration Procedure}

The coordinate expressions of equations of motion are:

$$
\begin{aligned}
\dot{M}_{1} & =\left(a_{3}-a_{1}\right) M_{2} M_{3}+a_{13} M_{1} M_{2}+\left(b_{3}-b_{1}\right)\left(M_{2} p_{3}+M_{3} p_{2}\right)+\left(c_{3}-c_{1}\right) p_{2} p_{3} \\
\dot{M}_{2} & =\left(a_{1}-a_{3}\right) M_{3} M_{1}+a_{13}\left(M_{3}^{2}-M_{1}^{2}\right)+\left(b_{1}-b_{3}\right)\left(M_{3} p_{1}+M_{1} p_{3}\right)+\left(c_{1}-c_{3}\right) p_{3} p_{1} \\
\dot{M}_{3} & =-a_{13} M_{2} M_{3} \\
\dot{p}_{1} & =a_{3} p_{2} M_{3}-a_{1} p_{3} M_{2}+a_{13} p_{2} M_{1}+\left(b_{3}-b_{1}\right) p_{2} p_{3} \\
\dot{p}_{2} & =a_{1} p_{3} M_{1}-a_{3} p_{1} M_{3}+a_{13} p_{3} M_{3}-a_{13} p_{1} M_{1}+\left(b_{1}-b_{3}\right) p_{3} p_{1} \\
\dot{p}_{3} & =a_{1}\left(p_{1} M_{2}-p_{2} M_{1}\right)-a_{13} p_{2} M_{3} .
\end{aligned}
$$

First integrals and invariant relation are:

$$
H=F_{1}, \quad F_{2}=M_{1} p_{1}+M_{2} p_{2}+M_{3} p_{3}, \quad F_{3}=p_{1}^{2}+p_{2}^{2}+p_{3}^{2}, \quad M_{3}=0 .
$$

Similarly as in the case of integration of Kirchhoff case, let us introduce new coordinates $\varphi, \psi$ according to the formulae:

$$
M_{1}=\sigma \cos (\varphi+\psi), M_{2}=\sigma \sin (\varphi+\psi), \quad p_{1}=s \cos \varphi, p_{2}=s \sin \varphi
$$

Using that

$$
\begin{aligned}
M_{1}^{2}+M_{2}^{2}= & \sigma^{2}, p_{1}^{2}+p_{2}^{2}=s^{2}, M_{2} p_{1}-M_{1} p_{2}=\sigma s \sin \psi, \\
& M_{1} p_{1}+M_{2} p_{2}=\sigma s \cos \psi,
\end{aligned}
$$

and having in mind that $M_{3}=0$ the first integrals become:

$$
\begin{aligned}
2 F_{1} & =a_{1} \sigma^{2}+2 b_{1} \sigma s \cos \psi+c_{1} s^{2}+c_{3} p_{3}^{2} \\
F_{2} & =\sigma s \cos \psi, \quad F_{3}=s^{2}+p_{3}^{2}, \quad F_{4}=0 .
\end{aligned}
$$

By squaring the last equation of (6.4) one gets

$$
\left(\dot{p}_{3}\right)^{2}=a_{1}^{2}\left(\sigma^{2} s^{2}-\sigma^{2} s^{2} \cos ^{2} \psi\right) .
$$


Using the expression of integrals (6.5) one gets

$$
\begin{aligned}
\dot{p}_{3}^{2}= & a_{1}\left\{\left(c_{1}-c_{3}\right) p_{3}^{4}+p_{3}^{2}\left(2\left(c_{1}-c_{3}\right) F_{3}+2 b_{1} F_{2}-2 F_{1}\right)\right. \\
& \left.+2 F_{1} F_{3}-2 b_{1} F_{2} F_{3}-c_{1} F_{3}^{2}-a_{1} F_{2}^{2}\right\}
\end{aligned}
$$

So $p_{3}$ is elliptic function of time. From (6.5) one can also find $\sigma(t), s(t)$ and $\psi(t)$. For complete integration one needs to find $\varphi$ as a function of time. By differentiation of $\tan \varphi=\frac{p_{2}}{p_{1}}$ using $s^{2}=p_{1}^{2} / \cos ^{2} \varphi$ one gets the additional differential equation

$$
\dot{\varphi}=\frac{a_{1} p_{3}(t) \sigma(t)}{s(t)}+\left(b_{1}-b_{3}\right) p_{3}(t)-a_{13} \sigma(t) \cos (\varphi+\psi(t))
$$

Introducing $u=\tan \frac{\varphi+\psi(t)}{2}$ the last differential equation leads to the Riccati equation

$$
\dot{u}=[f(t)+g(t)] u^{2}+[f(t)-g(t)] .
$$

Here $f(t)$ and $g(t)$ are known function of time given by:

$$
f(t)=\frac{1}{2}\left[\dot{\psi}(t)+\frac{a_{1} \sigma(t)}{s(t)} p_{3}(t)+\left(b_{1}-b_{3}\right) p_{3}(t)-a_{13} \sigma(t)\right], \quad g(t)=\frac{a_{13} \sigma(t)}{2}
$$

So, the integration procedure of the Chaplygin case leads to an elliptic integral (6.6) and an additional Riccati equation (6.7).

In the limit when $a_{13}$ goes to zero, the Chaplygin case becomes Kirchhoff case. Since in that case $g(t)=0$, the differential equation (6.7) can be solved by quadratures.

\subsubsection{Lax Representation for the Chaplygin Case}

The Kirchhoff case with an additional assumption $B=0$ can be regarded as a special case of the Clebsch case. In Perelomov (1981) Perelomov constructed a Lax representation for the Clebsch case as well as its higher-dimensional generalizations. As we have already mentioned, the Chaplygin case is a perturbation of the Clebsh case. Using that fact and using Perelomov's Lax representation from Perelomov (1981), a Lax representation for the Chaplygin's second case is constructed in Dragović and Gajić (2012):

Theorem 6.1 (Dragović and Gajić 2012) When $B=0$, on the invariant manifold given by the invariant relation, the equations of motion of the Chaplygin second case are equivalent to the matrix equation:

$$
\dot{L}(\lambda)=[L(\lambda), Q(\lambda)]
$$

where $L(\lambda)=\lambda^{2} L_{2}+\lambda L_{1}-L_{0}, Q(\lambda)=\lambda Q_{1}+Q_{0}$, and

$$
L_{2}=\operatorname{diag}\left(c_{1} / a_{1}, c_{1} / a_{1}, c_{3} / a_{1}\right), Q_{1}=\operatorname{diag}\left(a_{1}, a_{1}, a_{3}\right)
$$




$$
\begin{gathered}
L_{1}=\left[\begin{array}{ccc}
0 & -M_{3} & M_{2} \\
M_{3} & 0 & -M_{1} \\
-M_{2} & M_{1} & 0
\end{array}\right] L_{0}=\Gamma \Gamma^{T} \\
Q_{0}=\left[\begin{array}{ccc}
0 & -a_{3} M_{3}-a_{13} M_{1} & a_{1} M_{2} \\
a_{3} M_{3}+a_{13} M_{1} & 0 & -a_{1} M_{1}-a_{13} M_{3} \\
-a_{1} M_{2} & a_{1} M_{1}+a_{13} M_{3} & 0
\end{array}\right]
\end{gathered}
$$

The spectral curve $\operatorname{det}(L(\lambda)-\mu \cdot 1)=0$ is

$$
\begin{aligned}
\mathcal{C} & : \mu^{3}+\mu^{2} F_{3}-\lambda_{1}^{2} \mu^{2}\left(c_{3}+2 c_{1}\right) \\
& +\lambda_{1}^{2} \mu\left[2 F_{1}-\left(2 c_{1}+c_{3}\right) F_{3}\right]+\lambda_{1}^{4} \mu c_{1}\left(c_{1}+2 c_{3}\right) \\
& -\lambda_{1}^{6} c_{1}^{2} c_{3}-\lambda_{1}^{4}\left(2 c_{1} F_{1}-c_{1}\left(c_{1}+c_{3}\right) F_{3}\right)+\lambda_{1}^{2} a_{1} F_{2}^{2}=0,
\end{aligned}
$$

where $\lambda_{1}=\frac{\lambda}{\sqrt{a_{1}}}$. It is singular and has an involution $\sigma:\left(\lambda_{1}, \mu\right) \rightarrow\left(-\lambda_{1}, \mu\right)$. The curve $\mathcal{C}_{1}=\mathcal{C} / \sigma$ is a nonsingular genus one curve.

\subsection{Four-Dimensional Kirchhoff and Chaplygin Cases}

In Dragović and Gajić (2012) the four-dimensional generalizations of the Kirchhoff and Chaplygin cases are constructed on $e(4)$.

Let us consider the Hamiltonian equations with the Hamiltonian function:

$$
2 H=\sum A_{i j k l} M_{i j} M_{k l}+2 \sum B_{i j k} M_{i j} \Gamma_{k}+\sum C_{k l} \Gamma_{k} \Gamma_{l}
$$

in the standard Lie-Poisson structure on $e(4)$ given by:

$$
\begin{gathered}
\left\{M_{i j}, M_{k l}\right\}=\delta_{i k} M_{j l}+\delta_{j l} M_{i k}-\delta_{i l} M_{j k}-\delta_{j k} M_{i l} \\
\left\{M_{i j}, \Gamma_{k}\right\}=\delta_{i k} \Gamma_{j}-\delta_{j k} \Gamma_{i}
\end{gathered}
$$

A four-dimensional Kirchhoff case should have two linear first integrals: $M_{12}$ and $M_{34}$. It is interesting that under such an assumption, the "mixed" term in the Hamiltonian is missing.

Proposition 6.1 (Dragović and Gajić 2012) If $M_{12}$ and $M_{34}$ are the first integrals, then $B_{i j k}=0$.

The proof follows through direct calculations.

Definition 6.1 The four-dimensional Kirchhoff case is defined by

$$
\begin{aligned}
& 2 H_{K}=A_{1212} M_{12}^{2}+A_{1313}\left(M_{13}^{2}+M_{14}^{2}+M_{23}^{2}+M_{24}^{2}\right)+A_{3434} M_{34}^{2}+ \\
& A_{1234} M_{12} M_{34}+C_{11}\left(\Gamma_{1}^{2}+\Gamma_{2}^{2}\right)+C_{33}\left(\Gamma_{3}^{2}+\Gamma_{4}^{2}\right)
\end{aligned}
$$


On $e(4)$ the standard Lie - Poisson structure has two Casimir functions:

$$
\begin{aligned}
F_{1}= & \Gamma_{1}^{2}+\Gamma_{2}^{2}+\Gamma_{3}^{2}+\Gamma_{4}^{2}, \\
F_{2}= & \left(M_{13} \Gamma_{4}-M_{14} \Gamma_{3}+M_{34} \Gamma_{1}\right)^{2}+\left(M_{23} \Gamma_{1}+M_{12} \Gamma_{3}-M_{13} \Gamma_{2}\right)^{2} \\
& +\left(M_{24} \Gamma_{1}-M_{14} \Gamma_{2}+M_{12} \Gamma_{4}\right)^{2}+\left(M_{23} \Gamma_{4}+M_{34} \Gamma_{2}-M_{24} \Gamma_{3}\right)^{2}
\end{aligned}
$$

consequently, the general symplectic leaves are 8-dimensional. For complete integrability one needs four first integrals in involution. In Dragović and Gajić (2012) it is proved that except Hamiltonian, the four-dimensional Kirchhoff case has two linear first integrals $F_{3}=M_{12}, F_{4}=M_{34}$ and one additional quadratic first integral:

$$
\begin{aligned}
F_{5}= & a_{1}\left(M_{12} M_{34}+M_{14} M_{23}-M_{13} M_{24}\right)^{2} \\
& -c_{1}\left(\left(M_{13} \Gamma_{4}-M_{14} \Gamma_{3}+M_{34} \Gamma_{1}\right)^{2}+\left(M_{23} \Gamma_{4}+M_{34} \Gamma_{2}-M_{24} \Gamma_{3}\right)^{2}\right) \\
& -c_{3}\left(\left(M_{23} \Gamma_{1}+M_{12} \Gamma_{3}-M_{13} \Gamma_{2}\right)^{2}+\left(M_{24} \Gamma_{1}-M_{14} \Gamma_{2}+M_{12} \Gamma_{4}\right)^{2}\right)
\end{aligned}
$$

So, we have

Theorem 6.2 (Dragović and Gajić 2012) The four dimensional Kirchhoff case is completely integrable in the Liouville sense.

The final step in our program is construction of the four-dimensional analogue of the Chaplygin case. One may naturally expect that $M_{12}$ and $M_{34}$ would appear as the invariant relations. Starting from this assumption, we propose:

Definition 6.2 (Dragović and Gajić 2012) The four-dimensional Chaplygin case of the Kirchhoff equations on $e(4)$ is defined by the Hamiltonian:

$$
\begin{aligned}
2 H_{C h}= & A_{1212} M_{12}^{2}+A_{1313}\left(M_{13}^{2}+M_{14}^{2}+M_{23}^{2}+M_{24}^{2}\right)+A_{3434} M_{34}^{2} \\
& +A_{1234} M_{12} M_{34}+A_{1213} M_{12} M_{13}+A_{1214} M_{12} M_{14} \\
& +A_{1223} M_{12} M_{23}+A_{1224} M_{12} M_{24}+A_{1334} M_{13} M_{34} \\
& +A_{1434} M_{14} M_{34}+A_{2334} M_{23} M_{34}+A_{2434} M_{24} M_{34} \\
& +B_{121} M_{12} \Gamma_{1}+B_{122} M_{12} \Gamma_{2}+B_{123} M_{12} \Gamma_{3}+B_{124} M_{12} \Gamma_{4} \\
& +B_{341} M_{34} \Gamma_{1}+B_{342} M_{34} \Gamma_{2}+B_{343} M_{34} \Gamma_{3}+B_{344} M_{34} \Gamma_{4} \\
& +C_{11}\left(\Gamma_{1}^{2}+\Gamma_{2}^{2}\right)+C_{33}\left(\Gamma_{3}^{2}+\Gamma_{4}^{2}\right) .
\end{aligned}
$$

One can easily check that in this case $M_{12}$ and $M_{34}$ are indeed the invariant relations. Thus, we have constructed a system which is a four-dimensional analogue of the Chaplygin case. At the same time, the obtained system plays the role for the case of the Kirchhoff equations on $e(4)$ analogue to the role of the four-dimensional HessAppel'rot system for the Euler-Poisson equations on $s o(4) \times s o(4)$.

The rigid body dynamics is one of the very basic classical problems with enormous range of applications. The connections with several other areas of mathematics and mechanics such as the theory of Lie groups and algebras, differential and algebraic 
geometry, fluid mechanics etc. reflect its importance. There are still many important unsolved problems. Among those concerning integrability, we can mention in particular the question of classification of integrable cases of the Kirchhoff equations. As it was presented above, there is an amazing parallelism between the known integrable cases of the Euler-Poisson equations and the Kirchhoff equations. Nevertheless, the theory related to the Kirchhoff equations is far from being finalized as it is the case for the Euler-Poisson equations.

Acknowledgments The research was partially supported by the Serbian Ministry of Education and Science, Project 174020 Geometry and Topology of Manifolds, Classical Mechanics and Integrable Dynamical Systems. The authors would like to thank to the referees for several helpful comments and suggestions which significantly improved the presentation.

\section{References}

Adler, M., van Moerbeke, P.: Linearization of Hamiltonian systems, Jacobi varieties and representation theory. Adv. Math. 38, 318-379 (1980)

Adler, M., van Moerbeke, P., Vanhaecke, P.: Algebraic Integrability, Painlevé Geometry and Lie Algebras. A Series of Modern Surveys in Mathematics, vol. 47. Springer, Berlin (2004)

Andjelić, T., Stojanović, R.: Rational Mechanics. Zavod za izdavanje udžbenika, Belgrade (1966). [in Serbian]

Appel'rot, G.G.: Zadacha o dvizhenii tyazhelogo tverdogo tela okolo nepodvizhnoŭ tochki. Uchenye Zap. Mosk. Univ. Otdel. Fiz. Mat. Nauk. 11, 1-112 (1894)

Appel'rot, G.: About the first paragraph of the paper S. Kowalevski: Sur le proble 'me de la rotation d'un corps solide autour d'un point fixe (Acta Mathematica. 12:2). Matem. Sb. 16(3), 483-507 (1892)

Arkhangel'skiy, Y.A.: Analytical Dynamics of Rigid Body. Nauka, Moskva (1977). [in Russian]

Arnold, V.I.: Mathematical Methods of Classical Mechanics, Nauka, Moscow, (1974) [in Russian]. Springer, English translation (1988)

Arnold, V.I.: Sur la géométrie différentiale des groupes de Lie de dimension infinite et ses applications à l'hydrodinamique des fluides parfaits. Ann. Inst. Fourier (Grenoble) 16(fasc. 1), 319-361 (1966)

Arnold, V.I.: On a theorem of Liouville concerning integrable problems of dynamics. Siberian Math. J. 4(2), 471-474 (1963)

Arnold, V.I., Kozlov, V.V., Neishtadt, A.I.: Mathematical Aspects of Classical and Celestial Mechanics. URSS, Moscow (2009). [in Russian]

Audin, M.: Spinning Tops. A Course on Integrable Systems. Cambridge Studies in Advanced Mathematics, vol. 51. Cambridge University Press, Cambridge (1996)

Barkin, Y.V., Borisov, A.V.: Non-integrability of the Kirchhoff equations and related problems in rigid body dynamics. VINITI, No. 5037-B89 (1989)

Belokolos, E.D., Bobenko, A.I., Enol'skii, V.Z., Its, A.R., Matveev, V.B.: Algebro-geometric Approach to Nonlinear Integrable Equations. Springer Series in Nonlinear Dynamics, Springer-Verlag, Berlin Heidelberg (1994)

Belyaev, A.V.: On the motion of a multidimensional body with fixed point in a gravitational field. Mat. Sb. (N.S.), 114(156)(3), 465-470 (1981)

Belyaev, A.V.: On the general solution of the problem of the motion of a heavy rigid body in the Hess case. Mat. Sb. (N.S.), 206(5), 5-34 (2015)

Bilimović, A.: Rigid Body Dynamics. Mathematical Institute SANU, Special Editions (1955). [in Serbian]

Bobenko, A.I., Reyman, A.G., Semenov-Tien-Shansky, M.A.: The Kowalewski top 99 years later. Commun. Math. Phys. 122, 321-354 (1989)

Bogoyavlensky, O.I.: Integrable Euler equations on Lie algebras arising in physical problems. Soviet Acad. Izvestya 48, 883-938 (1984)

Borisov, A.V.: Necessary and sufficient conditions of Kirchhoff equation integrability. Regul. Chaot. Dyn. 1(2), 61-76 (1996)

Borisov, A.V., Mamaev, I.S.: Rigid Body Dynamics. Regul. Chaotic Dyn, Moscow-Izhevsk (2001). [in Russian] 
Borisov, A.V., Mamaev, I.S.: The Hess case in the dynamics of a rigid body. Prikl. Mat. Mekh. 67(2), 256-265 (2003)

Buchstaber, V.M.: Functional equations, associated with addition theorems for elliptic functions, and twovalued algebraic groups. Russian Math. Surv. 45, 213-214 (1990)

Buchstaber, V.M., Novikov, S.P.: Formal groups, power systems and Adams operators. Mat. Sb. (N. S) 84(126), 81-118 (1971). [in Russian]

Buchstaber, V.M., Veselov, A.P.: Integrable correspondences and algebraic representations of multivalued groups. Int. Math. Res. Note. 1996(8):381-400 (1996)

Buchstaber, V.: n-Valued groups: theory and applications. Moscow Math. J. 6, 57-84 (2006)

Buchstaber, V.M., Dragović, V.: Two-valued groups, Kummer varieties and integrable billiards. arXiv:1011.2716

Chaplygin, S.A.: Selected Works. Nauka, Moscow (1976). [in Russian]

Darboux, G.: Principes de Géométrie Analytique. Gauthier-Villars, Paris (1917)

Dokshevich, A.I.: Mekhanika tverdogo tela. Naukova dumka, Kiev 6, 48-50 (1974)

Dragović, V.: Algebro-geometric integration in classical and statistical mechanics. Zb. Rad. (Beogr.) 11(19), 121-154 (2006)

Dragović, V.: Geometrization and generalization of the Kowalevski top. Commun. Math. Phys. 298, 37-64 (2010)

Dragović, V.: Pencils of conics and biquadratics, and integrability. In: Topology, Geometry, Integrable Systems, and Mathematical Physics: Novikov's Seminar 2012-2014, Am. Math Soc. Translations, Volume 234, 2014, 97-120, ISBN-10:1-4704-1871-1, ISBN-13:978-1-4704-1871-7

Dragović, V., Milinković, D.: Analysis on Manifolds. Mathematical Faculty, Belgrade (2003). [in Serbian]

Dragović, V., Radnović, M.: Poncelet Porisms and Beyond. Springer, Birkhauser (2011)

Dragović, V., Gajić, B.: An L-A pair for the Hess-Apel'rot system and a new integrable case for the Euler-Poisson equations on $s o(4) \times$ so(4). Proc. R. Soc. Edinburgh Sect. A 131, 845-855 (2001)

Dragović, V., Gajić, B.: The Lagrange bitop on $s o(4) \times s o(4)$ and geometry of Prym varieties. Am. J. Math. 126, 981-1004 (2004)

Dragović, V., Gajić, B.: Systems of Hess-Appel'rot type. Commun. Math. Phys 265, 397-435 (2006)

Dragović, V., Gajić, B.: Elliptic curves and a new construction of integrable systems. Reg. Chaotic Dyn. 14(4-5), 466-478 (2009)

Dragović, V., Gajić, B.: On the cases of Kirchhoff and Chaplygin of the Kirchhoff equations. Reg. Chaotic Dyn. 17(5), 431-438 (2012)

Dragović, V., Gajić, B.: Four-dimensional Grioli precession. Reg. Chaotic Dyn. 19(6), 656-662 (2014)

Dragović, V., Gajić, B., Jovanović, B.: Systems of Hess-Appel'rot type and Zhukovskii property. Int. J. Geom. Methods Mod. Phys. 6(8), 1253-1304 (2009)

Dragović, V., Kukić, K.: New examples of systems of the Kowalevski type. Reg. Chaotic Dyn. 16(5), 484-495 (2011)

Dragović, V., Kukić, K.: Systems of the Kowalevski type and discriminantly separable polynomials. Reg. Chaotic Dyn. 19(2), 162-184 (2014a)

Dragović, V., Kukić, K.: The Sokolov case, integrable Kirchhoff elasticae, and genus 2 theta-functions via discriminantly separable polynomials. Proc. Steklov Math. Inst. 286, 224-239 (2014b)

Dubrovin, B.A.: Completely integrable Hamiltonian systems, matrix operators and Abelian varieties. Funkc. Anal. Appl 11, 28-41 (1977). [in Russian]

Dubrovin, B.A.: Theta-functions and nonlinear equations. Uspekhi Math. Nauk 36, 11-80 (1981). [in Russian]

Dubrovin, B.A., Krichever, I.M., Novikov, S.P.: Integrable Systems I. Dynamical Systems IV. Springer, Berlin (2001)

Dubrovin, B.A., Matveev, V.B., Novikov, S.P.: Nonlinear equations of Kortever-de Fries type, finite zone linear operators and Abelian varieties. Uspekhi Math. Nauk 31, 55-136 (1976). [in Russian]

Euler, L.: Du mouvement de rotation des corps solides autour d'un axe variable. Mémoires de l'académie des sciences de Berlin, 14, 154-193 (E292) (1765)

Fedorov, Y.N., Kozlov, V.V.: Various aspects of $n$-dimensional rigid body dynamics. Dynamical systems in classical mechanics, Am. Math. Soc. Transl. Ser. 2, 168, Am. Math. Soc., Providence, RI, 141-171 (1995)

Frahm, F: Uber gewisse Differentialgleichungen. Math. Ann. 8, 35-44 (1874)

Gajić, B.: Integration of Euler-Poisson equations by algebro-geometric methods. PhD. Thesis, Belgrade (2002) [in Serbian] 
Gajić, B.: The rigid body dynamics: classical and algebro-geometric integration. Zb. Rad. (Beogr.) 16(24), 5-44 (2013)

Gashenenko, I., Gorr, G., Kovalev, A.: Classical Problems of the Rigid Body Dynamics. Naukova Dumka, Kiev (2012)

Gavrilov, L., Zhivkov, A.: The complex geometry of Lagrange top. L'Enseignement Mathématique 44, 133-170 (1998)

Golubev, V.V.: Lectures on Integration of the Equations of Motion of a Rigid Body About a Fixed Point, Moskva, Gostenhizdat, (1953) [in Russian]; English translation: Transl. Coronet Books, Philadelphia, PA (1953)

Gorr, G.V., Kudryashova, L.V., Stepanova, L.A.: Classical Problems of the Rigid Bidy Dynamics. Naukova Dumka, Development and Current State, Kiev (1978)

Grioli, G.: Esistenza e determinazione delle prezessioni regolari dinamicamente possibili per un solido pesante asimmetrico. Ann. Mat. Pura e Appl. 6(fasc. 3-4), 271-281 (1947)

Grushevsky, S., Krichever, I.: Integrable discrete Schrodinger equations and a characterization of Prym varieties by a pair of quadrisecants. Duke Math. J. 152, 317-371 (2010)

Hess, W.: Ueber die Euler'schen Bewegungsgleichungen und über eine neue particuläre Lösung des Problems der Bewegung eines starren Körpers um einen festen. Punkt. Math. Ann. 37, 178-180 (1890)

Husson, E.: Recherche des intégrales algébriques. Ann. Fac. Sci. Toulouse 8, 73-152 (1906)

Jovanović, B.: Partial reduction of Hamiltonian flows and Hess-Appelrot systems on SO(n). Nonlinearity 20, 221-240 (2007)

Jovanović, B.: Symmetries and integrability. Publ. Inst. Math. Nouv. Ser. 84(98), 1-36 (2008)

Jurdjevic, V.: Integrable Hamiltonian systems on Lie Groups: Kowalevski type. Ann. Math. 150, 605-644 (1999a)

Jurdjevic, V.: Optimal Control, Geometry, and Mechanics. Mathematical Control Theory. Springer, New York (1999b)

Kharlamov, P.V.: Lectures on the Rigid Body Dynamics. Novosibirsk, p. 221 (1965)

Kharlamov, P.V.: On invariant relations of a sustem of differentrial equation. Mekh. Tverd. Tela N6, 15-24 (1974)

Kharlamov, P.V.: On algebraic invariant relations of the equations of motion of a rigid body having a fixed point. Mekh. Tverd. Tela N6, 25-33 (1974)

Kharlamov, P.V., Kovalev, A.M.: Invariant relation method in multibody dynamics. In: Proceedings of the Second World Congress of Nonlinear Analysis, Part 6 (Athens, 1996), Nonlinear Anal., 30(6) 3817-3828 (1997)

Kirchhoff, G.R.: Vorlesungen über Mathematische Physik. Mechanik, Leipzig (1874)

Komarov, I.V.: Kowalevski top for the hydrogen atom. Theor. Math. Phys. 47(1), 67-72 (1981)

Komarov, I.V., Kuznetsov, V.B.: Kowalevski top on the Lie algebras o(4), e(3) and o(3, 1). J. Phys. A 23(6), 841-846 (1990)

Komarov, I.V., Sokolov, V.V., Tsiganov, A.V.: Poisson maps and integrable deformations of the Kowalevski top. J. Phys. A 36, 8035-8048 (2003)

Kotter, F.: Sur le cas traite par M-me Kowalevski de rotation d'un corps solide autour d'un point fixe. Acta Math. 17, 209-263 (1893)

Kowalevski, S.: Sur le problème de la rotation d'un corps solide autour d'un point fixe. Acta Math. 12, 177-232 (1889)

Kozlov, V.V.: The nonexistence of an additional analytic integral in the problem of the motion of a nonsymmetric heavy solid around a fixed point, Vestnik Moskov. Univ. Ser. I Mat. Meh. 30(1), 105-110 (1975)

Kozlov, V.V.: Methods of Qualitative Analysis in the Dynamics of a Rigid Body. MGU, Moscow (1980). [in Russian]

Kozlov, V.V.: Symmetry Topology and Resonant in Hamiltonian Mechanics. Udmurt State University, Izhevsk (1995). [in Russian]

Kozlov, V.V., Onischenko, D.A.: Nonintegrability of Kirchhoff equations. Dokl. A. N. SSSR 266(6), 12981300 (1982). [in Russian]

Kozlov, V.V., Treschev, D.V.: Nonintegrability of the general problem of rotation of a dynamically symmetric heavy rigid body with a fixed point I. Vestn. Mosk. Univ., Ser. 1. Matem., Mekh. 6, 73-81 (1985) [in Russian] 
Kozlov, V.V., Treschev, D.V.: Nonintegrability of the general problem of rotation of a dynamically symmetric heavy rigid body with a fixed point II. Vestn. Mosk. Univ., Ser. 1. Matem., Mekh. 1, 39-44 (1986) [in Russian]

Lagrange, J.L.: Mécanique Analytique. Paris (1788)

Leimanis, E.: The General Problem of the Motion of Coupled Rigid Bodies about a Fixed Point. Springer, New York (1965)

Lubowiecki, P., Żołądek, H.: The Hess-Appelrot system. I. Invariant torus and its normal hyperbolicity.J. Geom. Mech. 4(4), 443-467 (2012a)

Lubowiecki, P., Żołądek, H.: The Hess-Appelrot system. II. Perturtbation and limit cycles. J. Differ. Equ. 252, 1701-1722 (2012b)

Manakov, S.V.: Remarks on the integrals of the Euler equations of the $n$-dimensional heavy top. Funkc. Anal. Appl. 10, 93-94 (1976). [in Russian]

Mumford, D.: Prym Varieties 1. A Collection of Papers Dedicated to Lipman Bers. Acad. Press, New York (1974)

Perelomov, A.M.: Some remarks on the integrability of the equations of motion of a rigid body in an ideal fluid. Funkc. Anal. Appl. 15, 83-85 (1981). [in Russian]

Ratiu, T.: Euler-Poisson equation on Lie algebras and the N-dimensional heavy rigid body. Am. J. Math. 104, 409-448 (1982)

Ratiu, T., van Moerbeke, P.: The Lagrange rigid body motion. Ann. Inst. Fourier Grenoble 32, 211-234 (1982)

Rubanovskii, V.N.: On a new particular solution of the equations of motion of a heavy solid in a liquid. Prikl. Matem. Mekhan 49(2), 212-219 (1985). [in Russian]

Sadetov, T.S.: The fourth algebraic integral of Kirchhoffs equations. J. Appl. Math. Mech. 64(2), 229-242 (2000)

Schottky,F.: Über das analytische Problem der Rotation eines starren Körpers im Raume von vier Dimensionen. Sitzungber. König. Preuss. Akad. Wiss. zu Berlin, pp. 227-232 (1891)

Simić, S.: On Noetherian approach to integrable cases of the motion of heavy top. Bulletin T.CXXI de l'Académie Serbe des Sciences et des Arts, Classe de Sciences mathématiques et naturelles, Sciences mathématiques, 25, 133-156 (2000)

Sokolov, V.V.: A new integrable case for Kirchoff equation. Theor. Math. Phys. 129(1), 1335-1340 (2001)

Sokolov, V.V.: Generalized Kowalevski Top: New Integrable Cases on $e(3)$ and so(4), in the Book the Kowalevski poperty, edt, vol. B, p. 307. AMS, Kuznetsov (2002)

Sokolov, V.V., Tsiganov, A.V.: Lax pairs for the deformed Kowalevski and Goryachev-Chaplygin tops. Theor. Math. Phys 131(1), 543-549 (2001)

Trofimov, V.V., Fomenko, A.T.: Algebra and Geometry of Integrable Hamiltonian Differential Equations. Moscow, copublished with Izdatel'stvo Udmurtskogo Universiteta, Izhevsk, Faktorial (1995)

Whittaker, E.T.: A Treatise on the Analytical Dynamics of Particles and Rigid Bodies, p. 456. Cambridge at the University Press, Cambridge (1952)

Weil, A.: Euler and the Jacobians of elliptic curves, in Arithmetics and Geometry, Vol. 1, Progr. Math. 35, Birkhauser, pp. 353-359. Mass, Boston (1983)

Ziglin, S.L.: The absence of an additional real-analytic first integral in some problems of dynamics. Funct. Anal. Appl. 31(1), 3-9 (1997)

Zhukovski, N.E.: Geometrische interpretation des Hess'schen Falles der Bewegung Eines Schweren Starren Korpers um Einen Festen Punkt. Jber. Deutschen Math. Verein. 3, 62-70 (1894) 